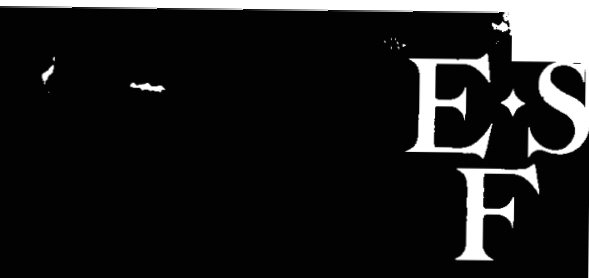

\title{
United States and Western Europe Cooperation in Planetary Exploration
}

(NASA-CB-184941) OAITED STAIES AVD UESTEB EUEOPE CCOPEEATIC IH EIABETLY EXELORATIOA (Eaticnal acadeny of sciences - lational Eesearch Council) $212 \mathrm{~F}$ CSCL 05A

$$
489-25776
$$

$\begin{array}{ll}63 / 84 & \text { Onclas } \\ 0214651\end{array}$ 


\title{
UNITED STATES AND WESTERN EUROPE COOPERATION IN PLANETARY EXPLORATION
}

\author{
Report of the \\ Joint Working Group \\ on Cooperation in Planetary Exploration
}

Space Science Board

National Research Council/

National Academy of Sciences
Space Science Committee

European Science Foundation

NATIONAL ACADEMY PRESS

Washington, D.C. 1986 
Notice by the National Research Council: The project that is the subject of this report was approved by the Governing Board of the National Research Council, whose members are drawn from the councils of the National Academy of Sciences, the National Academy of Engineering, and the Institute of Medicine. The members of the committee responsible for the report were chosen for their special competences and with regard for appropriate balance.

This report has been reviewed by a group other than the authors according to procedures approved by a Report Review Committee consisting of members of the National Academy of Sciences, the National Academy of Engineering, and the Institute of Medicine.

The National Research Council was established by the National Academy of Sciences in 1916 to associate the broad community of science and technology with the Academy's purposes of furthering knowledge and of advising the federal government. The Council operates in accordance with general policies determined by the Academy under the authority of its congressional charter of 1863 , which establishes the Academy as a private, nonprofit, self-governing membership corporation. The Council has become the principal operating agency of both the National Academy of Sciences and the National Academy of Engineering in the conduct of their services to the government, the public, and the scientific and engineering communities. It is administered jointly by both Academies and the Institute of Medicine. The National Academy of Engineering and the Institute of Medicine were established in 1964 and 1970, respectively, under the charter of the National Academy of Sciences.

Notice by the European Science Foundation: The Report has been reviewed according to the procedures of the Space Science Committee of the European Science Foundation (ESF). The Report was approved by the ESF Executive Council in March 1984.

The European Science Foundation was established in 1974 to act as a coordinator for the major Western European academies and research councils which support scientific research at a national level. Although these are funded mainly by governments, the ESF itself is a nongovernmental organization.

The principal objects of the Foundation are to advance cooperation in basic research; to promote mobility of research workers; to assist the free flow of information and ideas; and to facilitate the harmonization of the basic research activities supported by Member Organizations.

This study was supported by Contract NASW-3482 between the National Academy of Sciences and the National Aeronautics and Space Administration.

Copies available from:

Space Science Board

2101 Constitution Avenue, N.W.

Washington, D.C. 20418

Space Science Committee

1, Quai Lezay-Marnesia

67000 Strasbourg, France

Printed in the United States of America 


\section{THE JOINT WORKING GROUP ON COOPERATION IN PLANETARY EXPLORATION}

The United States Delegation

Eugene H. Levy

Head of the U.S. Delegation

Department of Planetary

Sciences

University of Arizona

Donald M. Hunten

Department of Planetary Sciences

University of Arizona

Tucson, Arizona

Harold Masursky

Branch of Astrogeology Studies

U.S. Geological Survey

Flagstaff, Arizona

Frederick L. Scarf

TRW Space Systems Group

Redondo Beach, California

Sean C. Solomon

Department of Earth, Atmospheric, and Planetary Sciences

Massachusetts Institute of Technology

Cambridge, Massachusetts

Laurel L. Wilkening

Vice-President for Research and Dean of the Graduate Office University of Arizona

Tucson, Arizona
The European Delegation

Hugo Fechtig

Head of the European Delegation

Max-Planck-Institut für

Kernphysik

Heidelberg, FRG

Hans Balsiger

Physikalisches Institut

Universität Bern

Bern, Switzerland

Jacques Blamont

Centre National d'Etudes Spatiale

Paris, France

Marcello Fulchignoni

Istituto Astrofisica Spaziale

Reparto di Planetologia

Rome, Italy

S. Keith Runcorn

School of Physics

University of Newcastle upon Tyne

United Kingdom

Frederic W. Taylor

Department of Atmospheric Physics Clarendon Laboratory, Oxford United Kindgom 


\section{SPACE SCIENCE BOARD}

Thomas M. Donahue, University of Michigan, Chairman

Don L. Anderson, California Institute of Technology

D. James Baker, Joint Oceanographic Institution

Roger Blandford, California Institute of Technology

Jay M. Goldberg, University of Chicago

Donald N.B. Hall, University of Hawaii

Donald M. Hunten, University of Arizona

William Kaula, National Oceanic and Atmospheric Administration

Harold Klein, The University of Santa Clara

Stamatios Krimigis, Johns Hopkins University

Robert M. MacQueen, National Center for Atmospheric Research

Carl E. McIlwain, University of California at San Diego

Robert Pepin, University of Minnesota

Christopher Russell, University of California at Los Angeles Blair Savage, University of Wisconsin

J. William Schopf, University of California at Los Angeles

Darrell Strobel, Johns Hopkins University

Anthony L. Turkevich, University of Chicago

Rainer Weiss, Massachusetts Institute of Technology

NAS/NRC Staff

Dean P. Kastel, Staff Director

Ceres Rangos, Secretary 


\section{COMMISSION ON PHYSICAL SCIENCES, MATHEMATICS, AND RESOURCES}

Herbert Friedman, National Research Council, Chairman

Clarence R. Allen, California Institute of Technology

Thomas D. Barrow, Standard Oil Company, Ohio

Elkan R. Blout, Harvard Medical School

Bernard F. Burke, Massachusetts Institute of Technology

George F. Carrier, Harvard University

Charles L. Drake, Dartmouth College

Mildred S. Dresselhaus, Massachusetts Institute of Technology

Joseph L. Fisher, Office of the Governor of the Commonwealth of Virginia

James L. Fletcher, University of Pittsburgh

William A. Fowler, California Institute of Technology

Gerhart Friedlander, Brookhaven National Laboratory

Edward D. Goldberg, Scripps Institution of Oceanography

Mary L. Good, UOP, Inc.

J. Ross MacDonald, University of North Carolina at Chapel Hill

Thomas F. Malone, Saint Joseph College

Charles J. Mankin, Oklahoma Geological Survey

William D. Phillips, Mallinckrodt, Inc.

Robert E. Sievers, University of Colorado

John D. Spengler, Harvard School of Public Health

George W. Wetherill, Carnegie Institute

Raphael G. Kasper, Executive Director

Lawrence E. McCray, Associate Executive Director 


\section{EUROPEAN SCIENCE FOUNDATION/ SPACE SCIENCE COMMITTEE}

Johannes Geiss, Physikalisches Institut der Universität Bern, Chairman

J. E. Blamont, Centre National d'Etudes Spatiales, Paris Carl-Gunnar Falthammar, Royal Institute of Technology, Stockholm

A. Johnsson, University of Trondheim, Dragvoll, Norway Anne McLaren, Medical Research Council, London F. Mariani, Università "La Sapienza" di Roma

F. M. Neubauer, Universität zu Köln, Federal Republic of Germany

H. Tennekes, Royal Netherlands Meteorolgical Institute

J. Trumper, Max-Planck-Institut für extraterrestrische Physik

H. C. Van De Hulst, Sterrewacht Laboratorium, RaLeiden, Netherlands

U. Van Twembekke, Ecole Royale Militaire, Brussels

ESF Staff

Josip Hendekovic

Catherine Bour 


\section{CONTENTS}

PREFACE

ix

1. SUMMARY OF RECOMMENDATIONS

2. INTRODUCTION 6

3. SCIENTIFIC EXPLORATION OF THE PLANETS 10

4. PAST PLANETARY EXPLORATION PROGRAMS 13

5. EXISTING PLANS FOR THE FUTURE 16

6. CASE FOR INCREASING LEVEL OF NASA-ESA COOPERATION 25

7. CANDIDATE COOPERATIVE PROGRAMS 31

8. SPECIFIC RECOMMENDATIONS 37

APPENDIXES: WORKING PAPERS OF THE STUDY TEAMS

A. Inner Planet Missions 51

B. Outer Planet Missions $\quad 88$

$\begin{array}{ll}\text { C. Primitive Body Missions } & 129\end{array}$ 


\section{PREFACE}

\section{PRECEDING PAGE BLANK NOT FILMED}

For a decade a formal liaison relationship has existed between the U.S. National Academy of Sciences/National Research Council's Space Science Board and the European Science Foundation's Space Science Committee, which has provided a regular forum for the exchange of views on the substance and direction of the space sciences and the opportunity to jointly study future cooperative endeavors between the United States and Western Europe. Our joint efforts have resulted in three earlier study reports: $A n$ International Discussion of Space Observatories (1976); $X$-ray and Gamma-Ray Astronomy in the 1980's (1978); and An International Discussion on Research in Solar and Space Physics (1983).

In keeping with this tradition and acting on a joint request from Dr. Frank Press, President, National Academy of Sciences, and Professor Hubert Curien, President, European Science Foundation, representatives of the Space Science Board and the Space Science Committee met in June 1982 and developed the structure and terms of reference for this joint study, whose purpose was to define a new framework for cooperation in planetary exploration.

In agreeing to undertake this joint effort and approving its conclusions, we are recognizing that the nature of future cooperation in planetary exploration, in this case, and space exploration, generally, will undergo a significant change that will be characterized by truly joint enterprises between the United States and Western Europe. This change is driven largely by an expansion of the European space science program, the incorporation of long-range planning, and broad growth in the European technology base. On behalf of our science communities, we welcome this change and 
believe that it can lead to a level of cooperation that will be in the best interests of our respective countries.

We wish to acknowledge our gratitude to the members of the Joint Working Group and their Task Groups, and especially to the untiring efforts of the joint chairmen, E. H. Levy, University of Arizona, and H. Fechtig, Max-Planck-Institute, Heidelberg. To the respective planetary program offices of the European Space Agency and the National Aeronautics and Space Administration, we also wish to express our appreciation for their cooperation in making expertise and fiscal support available to the Joint Working Group.

Johannes Geiss

Chairman

Space Science Committee
Thomas M. Donahue

Chairman

Space Science Board 


\section{1 \\ SUMMARY OF RECOMMENDATIONS}

This document reports the recommendations of the Joint Working Group (JWG) on Cooperation in Planetary Exploration, which was formed under the auspices of the European Science Foundation and the U.S. National Academy of Sciences/National Research Council in 1982. Over the course of a year the JWG studied the potential benefits of cooperative planetary programs carried out jointly by the National Aeronautics and Space Administration (NASA) and the European Space Agency (ESA). In addition, the JWG examined the various approaches to cooperative programs, the associated problems, and the candidate cooperative missions. The work and the discussions could not have been brought to a successful conclusion without the close collaboration and support of both NASA and ESA. Nevertheless, the recommendations presented are those of the Joint Working Group alone.

Scientific research into the planetary system addresses questions that are of fundamental concern to human beings. Through our investigations of the planets, satellites, asteroids, and comets, we are gathering the clues that will enable us eventually to reconstruct the sequence of events that led to the formation and, ultimately, to the present state of the solar system. We find these clues in the detailed composition, structure, and morphology of solar system objects. We are learning to understand the physical and chemical processes that go on in the interior and at the surface of planets, the processes that determine their environments, and the limits of stability of these environments. This last is of growing 
significance in view of our increasing disturbances of the Earth's environment.

We expect to learn of the conditions that gave rise to the origin of life, and we expect to learn about the manifestation of physical laws under conditions not approached in terrestrial laboratories. The solar system is the largest laboratory into which we can physically penetrate. What we learn there can influence our understanding of phenomena as diverse as the geophysics of the Earth and the creation of nebulae across the universe.

The JWG concluded that planetary investigations continue to be among the most intellectually challenging and important areas of the basic scientific research. In addition, planetary space missions stimulate far-reaching developments in advanced technology, which bring large benefits to the societies that sponsor the technology. Although much research has resulted from the space science programs of the different space agencies, important advantages can be obtained by combining some of the resources of the U.S. and European programs to pursue common objectives that complement the independent programs. The JWG believes that planetary exploration is one of the scientific and technological endeavors that would profit most from combining the efforts of the western nations.

The JWG strongly believes that the cooperative projects recommended here are complementary to the independent planetary programs planned by the space agencies. It is also the view of the JWG that successful cooperative programs will best be realized by strong independent agencies that have their own rigorous programs of fundamental research. The recommendations contained here are not intended to supplant the independent planetary programs-either in progress or being planned-of the United States or Europe.

The JWG's recommended program builds on the successful missions from the recent past and complements the missions that the space agencies are contemplating as independent projects. Independent projects that are currently in progress include the ESA Giotto flyby of Comet Halley in 1986 as well as the NASA Galileo Probe and Orbiter mission to Jupiter and the NASA Venus Radar Mapper. The plans and ongoing planetary programs of the other nations-notably the Soviet Union-have been taken into account insofar as they are known. In particular, we have taken note of 
the continuing and intensive Soviet Venus program, as well as the Soviet's planned flyby mission to Comet Halley.

The JWG has also taken account of candidate missions for the near future that are under consideration in the independent space agency programs. These include the Mars Geoscience and Climatology Orbiter and the Comet Rendezvous missions from the NASA Solar System Exploration Committee's core program and the Kepler Mars Aeronomy Orbiter mission from the ESA program. The JWG also notes, although it is formally outside the purview of this study, that should both the NASA and ESA Mars orbiters be implemented on schedules that allow it, significant advantage could be gained through the coordination of these two projects.

The Joint Working Group recommends that a program of three missions be carried out as cooperative projects, shared approximately equally by NASA and ESA, by the turn of the century. The projects, listed in order of the recommended launch sequence, without implication of scientific priority, are the Titan Probe and Saturn Orbiter mission, the Multiple Asteroid Orbiter with solarelectric propulsion, and the Mars Surface Rover mission. Of these three projects, two-the Titan Probe and Saturn Orbiter and the Multiple Asteroid Orbiter-can be accommodated within the agency programs as currently conceived. That is to say, for each of the two projects, each agency's share of the costs is comparable to the cost of projects currently included in the independent programs. The other project-the Mars Surface Rover-is technically and scientifically more ambitious and will require a special joint commitment of the sponsoring governments over and above the agency programs as they stand at present.

In the remainder of this section we summarize the specific actions that our recommendations entail.

\section{SUMMARY OF RECOMMENDED SPECIFIC ACTIONS}

The JWG recommends that NASA and ESA proceed with the Titan Probe and Saturn Orbiter mission on a prompt schedule, taking advantage of the technology that was developed for the Galileo project, where appropriate, in order to investigate the large range of phenomena in the Saturn system, while a European-built probe studies Titan's unique atmosphere in detail. This project is 
one that has already been well studied, and aspects of it are being discussed as possible program initiatives in both Europe and the United States. The recommended mission would integrate these separately proposed programs into a thorough investigation of the Saturn system, the highest priority target of further outer planet exploration. The scientific objectives of this mission are described in Appendix B and are comparable in scope to those of the NASA Galileo mission to the Jupiter system. The JWG's recommendation does not extend with the same temporal priority to a mission substantially reduced in scope or to one addressing only a small part of the total science objectives.

The Multiple Asteroid Orbiter would utilize a low-thrust solarelectric propulsion system to temporarily orbit approximately six asteroids, selected to provide a general picture of this diverse class of bodies. The spacecraft would stay with each asteroid long enough to carry out detailed optical, chemical, physical, and mineralogical investigations and would determine the mass and mean density of each. The JWG notes the European interest in pursuing development of solar-electric propulsion. In order to enable this mission, the JWG recommends an early start on development of an operational propulsion system capable of carrying out this recommended mission. Also recommended is an early start on advanced development of instruments to accomplish the major science objectives of asteroid rendezvous. The JWG notes that solar-electric propulsion also has numerous additional applications to space activities, including use in a future comet-nucleus sample return mission.

The JWG recommends the Mars Surface Rover mission for detailed study of the geology, geochemistry, and geophysics of Martian terrains as well as of the planet's interior and atmosphere. The Mars Surface Rover mission would represent an excellent early step in a staged approach to the intensive investigation of Mars that would eventually include the identification and acquisition of Martian material in a future sample return mission. The JWG recommends that work begin to define a Mars Surface Rover and to develop instruments and experiments that can take advantage of the capabilities offered by a mobile laboratory on the Martian surface. 


\section{SCHEDULES AND PROGRAMMATIC IMPACTS}

Both of the recommended missions that can fit into the ongoing programs - the Titan-Saturn and the Multiple Asteroid Orbiter-are already being discussed by NASA and ESA as candidates for joint endeavors. The JWG recommends that the agencies proceed to implement these missions on a schedule that leads to launches in 1992 (for Titan-Saturn) and 1994 (for the Multiple Asteroid Orbiter).

The Mars Surface Rover requires an expansion in the currently limited scope of the planetary programs. Moreover, it would benefit from substantial advanced development efforts, both for the vehicle and for the scientific instruments. The JWG recommends that such development be started and that the Rover mission be implemented on a schedule leading to launch in 1996.

This schedule, extending to the end of the century, is constrained entirely by the limited fiscal resources of the present agency programs. Scientific and technical considerations do not dictate such a slow pace, and indeed, it would be advantageous if these missions were carried out earlier. The JWG would be urging a faster implementation were it not that it recognizes the need for political decisions to increase the pace of planetary science. 


\section{2 INTRODUCTION}

The Joint Working Group (JWG) on Cooperation in Planetary Exploration was established in the summer of 1982 by the Space Science Board of the U.S. National Academy of Sciences/National Research Council (NAS/NRC) and the Space Science Committee of the European Science Foundation. The JWG was formed for the purpose of putting forward a framework for a new level of U.S.-European cooperation in planetary exploration. The JWG was charged specifically with exploring the desirability of carrying out jointly planned and jointly executed space missions to planets and to primitive solar system bodies. The JWG was also required to examine the potential approaches to cooperation, to set forth the principles on which joint planetary science investigations are to be conducted, and in consultation with the National Aeronautics and Space Administration (NASA) and the European Space Agency (ESA), to formulate a strategy, including specific missions, that would constitute a program of such collaboration.

The start of these discussions was based on the belief that expanded cooperation, including jointly conducted missions of planetary exploration, can contribute significantly to the attainment of important scientific goals and offers potential benefits to both the United States and the European space science programs. There is a historical and natural alliance between Europe and the United States, and this expanded cooperation in planetary exploration would enhance the scientific and technological creativity of both. Jointly conducted missions would continue, at a higher level, the collaboration in space research that already has achieved many notable successes. 


\section{OVERVIEW OF THE CHARGE}

Specific issues to be addressed in the final recommendations were to include the following:

- types and levels of possible cooperative activities in the planetary sciences;

- definition of a desirable approach to European-U.S. cooperation;

- specific or general scientific areas that seem most promising as the main focus of cooperative efforts;

- potential mission candidates for cooperative ventures;

- identification of special issues or problems for resolution by negotiation between the agencies, and possible suggestions for their resolution;

- identification of coordinated technological and instrumental developments for planetary missions.

In addition to the general questions that are involved in establishing a close, fruitful, and continuing collaboration, the JWG identified specific criteria that should be satisfied by any mission that is a candidate to become a cooperative project:

- It should be naturally and readily divisible into two separate but scientifically integrated elements.

- Each element should require roughly equal resources for its development and realization.

- Each part should deal with scientific questions of the highest priority; the project should be one that readily will attract widespread support and endorsement within the planetary science communities and within other scientific and societal groups as well.

- It should involve a broad cross section of scientific disciplines in both Europe and the United States.

- Separation of the two elements should extend to the technical and management interfaces so as to ensure efficient implementation of the joint project.

- The scientific payloads, however, should be shared.

In addition,

- It is highly desirable that each element be technologically challenging. 
- Consideration should be given to the likelihood that the project represents one step in a sequence of projects developing a common theme.

\section{DESCRIPTION OF THE JOINT WORKING GROUP AND RESPONSE TO THE CHARGE}

In order to examine the possible projects effectively, the JWG set up three study teams, each of which was concerned with a particular area of solar system research: the terrestrial planets, the Saturn system, and the primitive bodies-asteroids and comets.

The study teams were instructed to examine missions at several levels of cost. At the lowest level, the teams studied missions with a total cost of about $\$ 400$ million (or 400 million accounting units). At this level the European share of a mission would fit within the ESA mandatory science program, and the U.S. share would be comparable to low-cost missions being contemplated in the NASA planetary core program. In addition, the study teams were asked to report on projects involving greater scientific and technological efforts and commensurably higher costs that, on the European side, could be implemented through the ESA optional science program. An important aspect of all of the mission studies was the identification of challenging technological advances in which both sides could participate.

\section{INSTITUTIONAL RELATIONSHIPS WITH ESA AND NASA}

Throughout these discussions both NASA and ESA have participated actively and have shared closely with JWG their views and perceptions. In the United States this process continues a long tradition of close collaboration between NASA and the NAS/NRC Space Science Board in formulating long-term scientific objectives and strategy for space science. In Europe, the European Science Foundation's Space Science Committee works as a coordinating body for scientific research. The committee has advised ESA on matters of general science policy and international cooperation, and it has contributed to the planning of space science in Europe and its coordination at the international level. 


\section{ORGANIZATION OF THE REPORT}

In the following report a brief overview of the goals and significance of planetary exploration is first given, followed by a review of the space age planetary programs. The existing planetary strategies and plans of the two agencies are then discussed and the case for expanded cooperative approaches to these endeavors is examined. Finally, the Joint Working Group's specific recommendations are set forth. The reports of the study teams are contained in the appendixes.

This report addresses only those aspects of the planetary programs that are tied closely to questions of cooperation. It is not intended to reconsider the many other aspects of planetary exploration that have been taken up by the Committee on Planetary and Lunar Exploration of the Space Science Board and by the European Science Foundation's Space Science Committee. 


\section{3 SCIENTIFIC EXPLORATION OF THE PLANETS}

Questions about the nature, behavior, and origin of this world have occupied man's thoughts since before recorded history, yet only in the second half of the twentieth century has the technological capability been developed-rocket propulsion, long-distance radio transmission, electronic automation of making a scientific exploration of the solar system. Today the entire system lies within reach of our scientific instruments.

Such a development did not occur in a vacuum but rather as part of a larger scientific and technological expansion that began several hundred years ago. We believe that we now have a firm grasp of the basic physical laws that determine the behavior of solar system objects and that governed the solar system's formation. However, the manifestation of these physical laws in natural systems is so complicated as to make it impossible, in practice, to deduce their effects. At every step in our exploration of the solar system we have been confronted with phenomena that tax our understanding and that lead to new insights about how the physical laws act to shape the world in which we live. The discovery of the complicated behaviors of real physical systems, and the discovery of the intricate mechanisms by which the simple physical laws produce macroscopic phenomena, is intrinsic to science and is as fundamental as the attempt to discover new physical laws.

Our scientific motivations for solar system exploration include the expectation that we shall gather clues about the processes and events that led from a diffuse assemblage of interstellar gas to the ordered system of the Sun and its surrounding planets. By 
coupling this detailed knowledge to astronomical observations of the more generalized aspects of star formation, we expect to gain a deeper understanding of the formation of stars and the prevalence of planetary systems. This expectation, and the others that motivate our drive to investigate the solar system, is supported by the many advances that already have resulted from our first exploratory forays into deep space.

We expect to discover the complex behavior of planetary objects, to learn what physical effects produce this behavior, and to be able to extrapolate that understanding into a generalized knowledge about our own planet Earth. Included here is the appreciation of the variability - and the instability - of terrestrialplanet environments that has grown out of our studies of Mars and Venus. A need to understand the causes of such variability is growing increasingly acute as human influences on the terrestrial environment become continually greater.

We expect to learn what events led to the benign, life-supporting environment on Earth and to define the nature of conditions in which life arose and evolved on this planet. By comparing the early history of Earth with that of other similar planets, we expect to advance our understanding of the possible prevalence of life-supporting planets. All of these investigations are critical to sharpening our picture of man's origin and to unravelling the grand mystery of his place in the universe.

Through our scientific explorations of the solar system, we gain first-hand knowledge about the physical phenomena that shape the behavior of large-scale natural systems. Far into the indefinite future the solar system and its nearest vicinity will represent the entire region of space into which we can probe and gain first-hand experience. In a real sense, the solar system is man's largest extended laboratory. The many phenomena that can be investigated there range from planetary tectonics to viscous disk systems and explosive, high-energy plasma dynamics.

Finally, the solar system is the largest sphere into which human activity can expand during any future time worthy of serious contemplation. Although it is premature to guess at the useful character and intensity of such human activity as it extends into space, it is worth noting that our scientific explorations of the solar system will generate that foundation of knowledge on which future decisions about other intensive activities will have to be made. 
In a broad historical perspective, bringing the entire solar system within reach of direct human experience will be one of the notable accomplishments of our time. It is a particularly appropriate goal for cooperation between the United States and Europe. 


\section{4 \\ PAST PLANETARY \\ EXPLORATION PROGRAMS}

Scientific exploration of the solar system has advanced through the application of complementary techniques beginning with optical astronomy. Such studies continue to provide new and important information, but the center of empirical planetary investigation has shifted toward in situ measurements carried out by scientific instruments deployed on spacecraft and planetary probes, and detailed laboratory studies of extraterrestrial material that has been collected and returned to Earth or that has fallen by chance to the Earth's surface.

The first phase of planetary exploration, made possible from development of space technology and new analytical tools, led to the discovery of new and previously unexpected phenomena. One need only point, for example, to lunar paleomagnetism, the volcanoes and river channels on Mars, the chemical and isotopic heterogeneity of solar system objects, the rings of Jupiter, and the extraordinary dynamical phenomena in Saturn's rings to glimpse the expanded and more accurate picture that today we have of the solar system. Solar system exploration has also been distinguished by triumphs of technological virtuosity: the Voyager missions to Jupiter and Saturn (and, one hopes, later to Uranus and Neptune), the Viking mission to Mars, the lunar sample returns, the Soviet Venus landings, and numerous other missions.

Discoveries and new perceptions that result from early planetary exploration led to the present focus on fundamental problems. Increasing emphasis is now cast on investigations designed to gather specific information, although it must be said that the 
rate at which unexpected new discoveries are made continues at an unabated pace.

In the past, planetary exploration programs were confined to two nations: the United States and the Soviet Union. Recently, the number of countries carrying out active planetary exploration programs has grown. The European Space Agency's (ESA's) Ariane rocket and its Giotto mission to Comet Halley mark the emergence of a significant independent European capability for deep-space investigation. At the same time, Japan is embarking on its first planetary, deep-space mission.

International cooperation has always been a feature of planetary exploration, and the level of cooperation seems to be on the increase. For instance, the Soviet Union distributed lunar samples for study in the international community, and the scheduled Soviet mission to Comet Halley in 1986 will involve an unprecedented number of scientists from other countries.

The U.S. space science program has a long tradition of being open to participation of scientists from other nations, of which the European scientific community in particular has taken advantage. For example, in the early analysis of lunar samples brought to Earth in the Apollo project, some 56 scientists, out of a total of 197, worked outside the United States. In addition, instruments built by European principal investigators have been a regular feature of U.S. space science missions - in the planetary as well as in the other parts of the U.S. program. European co-investigators are also commonly associated with U.S. space science experiments.

In the European space science program the Giotto mission to Comet Halley is the first planetary mission. The mission includes substantial participation by U.S. scientists, although not as principal investigators. Recently formulated policies, however, make provision for the international scientific community to have regular access in the future to flight opportunities on ESA missions. This is a welcome evolution; the Joint Working Group (JWG) looks forward to its implementation and notes the necessity for participating nations to make funds available to allow the development of instruments to take advantage of such flight opportunities.

Significant levels of cooperation have been achieved between the National Aeronautics and Space Administration (NASA) and various European countries. For example, the International Ultraviolet Explorer, the Infrared Astronomical Satellite, the ISEE-3 spacecraft, the Galileo project, Spacelab, and Space Telescope are 
among the projects that can be cited for significant components of U.S.-European cooperation.

This brief discussion would be unbalanced if it did not recall the attempt to institute a cooperative project along the lines of one of the models proposed in this report. The International SolarPolar mission failed to achieve its design goals when the United States unilaterally withdrew its spacecraft from the project in order to meet budgetary exigencies several years ago. Although the restructured project, with diminished U.S. participation, involved roughly equal contributions to the overall cost, such a precipitate abandonment of a project-planned and under way-is a poor precedent.

Successful international cooperation will require that the participating nations reliably maintain their commitments. A strong desire persists in the scientific communities for international cooperation in space science, even after the Solar-Polar experience. This testifies powerfully to the value of such cooperation by scientists on both sides of the Atlantic Ocean, as past experiences with cooperation in space science have proved advantageous to both sides. The more ambitiously symmetrical initiatives recommended in this report would work to the greater advantage of the participating nations and scientists. 


\section{5 EXISTING PLANS FOR THE FUTURE}

In the United States the space science program is constructed on the basis of long-term strategies that define the most important scientific objectives based on the major questions that space exploration seeks to answer and on the information gained by exploration already undertaken. The program is built so as to maintain a balance between the more deeply intensive investigations that follow initial exploration and new explorations of previously unexamined objects and regions, which have large potential for important discovery. The scientific strategies are constructed by the NAS/NRC Space Science Board, and then the detailed implementation plans are drawn and carried out by the National Aeronautics and Space Administration (NASA).

In Europe, for the most part, scientific space investigations have proceeded according to a different approach; until recently there has been no attempt to define an overall strategy for planetary exploration. Space science projects have been started at the initiative of independent and ad hoc groups of scientists, and the European Space Agency (ESA) has played the role of implementation agency.

Now that Europe has acquired its own substantial spacecraftlaunching capability and has started its first project in the planetary arena-the Giotto mission to Comet Halley-more attention has been paid to the longer term objectives and structure of planetary science. The Space Science Committee of the European Science Foundation has taken the first steps in formulating a European strategy for planetary exploration. 


\section{SCIENTIFIC STRATEGIES FOR PLANETARY EXPLORATION}

This chapter summarizes and describes the scientific objectives and the implementation plans for planetary exploration that have been put forward in both the United States and Europe. The recommendations of this Joint Working Group (JWG) are based on those objectives and plans. The JWG's ability to recommend the undertaking of major cooperative U.S.-European planetary projects arises, in large measure, from the commonality of interests, goals, and plans on the two sides of the Atlantic.

A long-term scientific strategy for the U.S. national program of solar system exploration was elaborated in three documents prepared, over a period of 5 years, by the Committee on Planetary and Lunar Exploration (COMPLEX) of the Space Science Board. These documents are Report on Space Science 1975, part II (1976), which presents a strategy for exploration of the outer planets during 1975-1985; Strategy for Exploration of the Inner Planets, 1977-1987 (1978); and Strategy for the Exploration of Primitive Solar-System Bodies-Asteroids, Comets, and Meteoroids: 19801990 (1980). In each report, recommendations are cast in the form of scientific objectives to be achieved rather than in the form of scientific missions to be flown. These strategies have provided long-term scientific guidance to NASA over the past 8-year period, a time during which severe budgetary restrictions and consequent developmental problems have forced several significant reductions and alterations in mission plans.

The European Science Foundation has formulated a European strategy for planetary exploration in its report Planetary Science in Europe-Present State and Outlook for the Future (1982), based on the conclusion of a workshop held in Strasbourg, France, September 15-18, 1980.

\section{Outer Planets}

The outer planet science objectives were formulated after the Pioneer 10 and 11 encounters with Jupiter and in anticipation of the Pioneer 11 Saturn flyby and the Voyager mission to Jupiter and Saturn. Because Jupiter is the largest planet of the solar system and demonstrates a wide range of dynamical and physical behavior, and because Uranus is the closest of a class of major 
planets-yet unstudied by spacecraft-containing large amounts of ice and rock, highest priority was assigned to an in-depth exploration of Jupiter and its satellites and initial reconnaissance of Uranus during 1975-1985. The primary objectives for Jupiter exploration, in order of importance, are the determination of (1) the chemical composition and physical state of its atmosphere, (2) the chemical composition and physical state of its satellites, and (3) the topology and behavior of its magnetic field and the energetic particle fluxes. NASA was also advised to anticipate the exploration of Saturn and its satellites, and these same objectives should apply to the intensive study of Saturn.

The primary objectives for reconnaissance of Uranus for the 1980 s are, in order of priority, (1) determining if there exists a planetary magnetic dipole field, its strength and orientation, (2) measurement of the quadruple gravitational moment of Uranus and the masses of the major satellites, (3) measurement of the gross morphologic structures of the planet and satellites, (4) compositional information and vertical and horizontal atmospheric structure at good resolution for Uranus, and (5) estimating the planetary heat source.

Principal future goals of the exploration of Saturn are, in order of importance, (1) intensive investigation of the atmosphere of Saturn, (2) determination of regional surface chemistry and properties of satellites and ring particles, (3) intensive investigation of Titan, and (4) determination of atmospheric dynamics and structure of Saturn. As this report is written, the scientific objectives for Saturn are being reexamined in the light of recent discoveries. The JWG's deliberations relied on the revised view of the Saturn system that is emerging from this study.

\section{Inner Planets}

The inner planet science objectives were formulated after the Viking missions to Mars and in anticipation of the Pioneer Venus mission. Because of the rich diversity of phenomena manifested by terrestrial planets with atmospheres, major advances in planetology can be achieved by the intensive exploration of these bodies. Consequently, it is recommended that the triad of terrestrial planets, Earth, Mars, and Venus, receive the major focus in exploration of the inner solar system for the decade 1977-1987. The ultimate goal is to understand the present state and evolution of terrestrial 
planets with atmospheres. The comparative planetology of these bodies is key to understanding the formation of the Earth, its atmosphere and oceans, and the physical and chemical conditions that lead to the origin and evolution of life. The atmospherefree terrestrial bodies, Mercury and the Moon, are regarded as complementary bodies of high scientific interest.

The primary objectives, in order of scientific priority, for the continued exploration of Mars are (1) the intensive study of local areas to

- establish the chemical, mineralogical, and petrological character of different components of the surface material, representative of the known diversity of the planet;

- establish the nature and chronology of the major surfaceforming processes;

- determine the distribution, abundance, and sources and sinks of volatile materials, including an assessment of the biological potential of the Martian environment, now and during past epochs; and

- establish the interaction of the surface material with the atmosphere and its radiation environment,

(2) the exploration of the structure and general circulation of the Martian atmosphere, (3) the study of the structure and dynamics of Mars' interior, (4) the establishment of the nature of the Martian magnetic field and the character of the upper atmosphere and its interaction with the solar wind, and (5) the establishment of the global chemical and physical characteristics of the Martian surface. These objectives are multiply connected.

The primary objectives of the exploration of Venus, beyond the Pioneer Venus mission, in order of importance are (1) to obtain a global map of the topography and morphology of its surface at sufficient resolution to allow identification of the gross processes that have shaped the surface, (2) to determine the major chemical and mineralogical composition of the surface material, (3) to determine the concentrations of photochemically active gases in the $65-$ to $135-\mathrm{km}$ altitude region, and (4) to investigate the physical and chemical interactions of the surface with the atmosphere and the composition and formation of atmospheric aerosols. 


\section{Primitive Bodies}

The primitive solar system bodies-asteroids and comets, which have not yet been visited by spacecraft-are thought to preserve a record of the earliest processes of solar system formation. An important aspect of these bodies is their diversity and the relationship of that diversity to variations in conditions of formation and evolutionary modifications.

The primary scientific objectives put forward for comet exploration during the next decade are, in order of priority, (1) to determine the composition and physical state of the nucleus (determination of the composition of both dust and gas is an important element of this objective), (2) to determine the processes that govern the composition and distribution of neutral and ionized species in the cometary atmosphere, and (3) to investigate the interaction between the solar wind and the cometary atmosphere. In view of the apparent diversity of comets, it is important to observe the changing state of the nucleus and coma of a comet during perihelion passage. A secondary objective is to determine the physical state of the cometary dust for the purpose of relating it to interplanetary and interstellar dust.

The primary scientific objectives for the exploration of asteroids are, in order of priority, (1) to determine their composition and bulk density, (2) to investigate the surface morphology, including evidence for endogenic and exogenic processes and evidence concerning interiors of precursor bodies, and (3) to determine the internal properties, including states of magnetization of several carefully chosen asteroids selected on the basis of their diversity. Measurement of asteroid composition and morphology must be carried out together, because the interpretation of composition variations and morphology of an asteroid are intimately connected.

\section{THE U.S. NATIONAL PROGRAM FOR PLANETARY EXPLORATION}

NASA has two ongoing missions to the outer planets. The Voyager 2 spacecraft is currently enroute to Uranus and will make scientific studies of that planet and its satellites; it will subsequently fly past Neptune. The Galileo Orbiter and Probe mission presently is scheduled to be launched in 1986 and to arrive at Jupiter in 1988. The Galileo probe will measure the composition 
of the Jupiter atmosphere, and the orbiter will conduct imaging and remote-sensing measurements of the planet and satellites and determine the characteristics of the Jovian magnetic field and magnetosphere. Successful completion of the Voyager and Galileo missions will address all of the primary objectives for outer planet exploration identified by COMPLEX for the decade 1975-1985.

At this writing, NASA has one currently approved mission to the inner planets-the Venus Radar Mapper (VRM). VRM will obtain, by high-resolution radar imaging, a geological map of Venus in order to determine the physical processes that shaped that planet's surface.

The large gap between the last two new starts of NASA in planetary exploration (Galileo in fiscal year (FY) 1978 and the Venus Radar Mapper in FY 1984) and the present is a consequence of several factors, including severe budgetary restrictions and programmatic postponements and project delays that attended the space shuttle's development, as well as the rising costs of proposed new missions. To identify a means of continuing planetary exploration in a time of constrained resources, NASA formed the Solar System Exploration Committee (SSEC), charged with the task of formulating a program of low-cost spacecraft missions that would address significant aspects of the scientific objectives identified in the COMPLEX strategy reports. The SSEC proposed a core program of planetary missions intended to establish and maintain a steady advance in our understanding of the solar system, yet to do so within a framework of cost-saving design and management elements. An important part of the core program is the establishment of the Planetary Observer Program-a constant level-ofeffort program consisting of inner planet missions accomplished with low-cost spacecraft using designs derived from existing Earth orbital vehicles. Another important element of the core program is the development of a modular spacecraft, the Mariner Mark II, which can be inexpensively reconfigured for different missions, even outside the inner solar system.

The SSEC recommended a core planetary program consisting of a sequence of missions, the first four of which are the Venus Radar Mapper (1988 launch), a Mars Geoscience and Climatology Orbiter (1990 launch), a Comet Rendezvous and Asteroid flyby (launch 1990-1992), and a Titan Probe and Radar Mapper mission. 
The first mission, which has been approved recently as a new start-the Venus Radar Mapper-will complete the global characterization of the surfaces of the two most Earth-like planets-Mars and Venus - thereby allowing significant comparative studies that will sharpen our understanding of the evolution of terrestrial planets.

The Mars Geoscience and Climatology Orbiter is the first of the new class of planetary observers. Two fundamental objectives are combined in this mission: the determination of the global surface composition of Mars and the determination of the role of water in the Martian climate.

The third initial core mission, the Comet Rendezvous and Asteroid flyby, will make use of the Mariner Mark II spacecraft. A rendezvous mission to a comet permits the detailed analysis of a cometary nucleus necessary to understanding its origin and evolution; the mission will produce a significant scientific return that is commensurate with the next step of comet exploration beyond that which will be taken by the fast flybys of Comet Halley in 1986.

The Titan Probe and Radar Mapper mission would use a modified Galileo probe together with a flyby or orbiter spacecraft equipped with a simple radar. Titan, the largest satellite of Saturn, is unique in having a thick atmosphere composed mostly of nitrogen, smaller but significant amounts of methane or possibly ethane, and perhaps argon. Titan has the only planetary atmosphere in the solar system that may be similar to Earth's before life arose. The atmosphere-thick with aerosols believed to be composed of organic compounds-obscures Titan's surface. The core Titan mission would determine the fundamental chemical composition of Titan's atmosphere and the nature of its unseen surface.

A number of candidate subsequent missions have been suggested. A Mars aeronomy orbiter would establish, with high precision, whether Mars has an internal magnetic field and would investigate the interaction of the planet's upper atmosphere and ionosphere with solar radiation and the solar wind. A Venus atmospheric probe would gather definitive information on the abundance of major and minor trace components of the Venus atmosphere, leading toward an understanding of conditions in the inner solar system at the time the planets accreted. A lunar geoscience 
orbiter would provide a global map of surface elemental and mineralogical composition, and other properties, and it would resolve speculations about the possible presence of condensed water and other volatiles in polar cold traps. A Mars surface probe mission would establish seismic, meteorological, and geoscience stations on the Martian surface. These would determine the level of Martian seismicity, provide surface weather data toward an understanding of its climatic pattern, and provide detailed geochemical analyses. A comet atomized sample return mission would provide elemental and isotopic composition analyses of gases and dust from the coma of a comet-data complementary to those acquired by a comet rendezvous mission. Ideally, the cometary material would be returned to terrestrial laboratories from the same comet observed by the rendezvous mission, thereby providing a detailed characterization of at least one such body. An Earth-approaching asteroid rendezvous mission would characterize in detail a selected number of this class of bodies. A Saturn orbiter would carry out investigations of the Saturnian satellites, ring systems, and magnetosphere. It would permit the first extended studies of the evolution of ring structures, as well as close-up examination of otherwise poorly seen satellites, and additional radar coverage of Titan's surface. Saturn and Uranus flyby probe missions would facilitate an in situ determination of the composition and structure of the Saturnian and Uranian atmospheres and clouds for comparison with Jupiter, which presumably will already have been investigated by the Galileo probe.

\section{THE EUROPEAN PROGRAM FOR PLANETARY EXPLORATION}

The European scientific community has had to take into account the relatively low budget that has been available for space missions. In the past this led to the decision that significant planetary exploration would be foregone in Europe. The European space science program was, until recently, limited to studies of the interplanetary medium and remote astronomical observations performed from Earth-orbiting spacecraft.

Even with the development of the Ariane space rocket, independent European planning has been restricted to low-budget missions with relatively modest ambitions. As demonstrated by the Giotto mission to Comet Halley, however, a smaller number 
of exciting planetary projects can be undertaken even within the tight restrictions. Future planning holds even more promise, especially in view of the opportunities offered by U.S.-European cooperative projects.

Among the planetary missions under discussion in Europe, at the time of the JWG deliberations, as possible candidates for implementation by ESA are the following:

- Polo, a lunar polar orbiter, would gather information to produce a map of chemical element abundances over the lunar surface, would obtain surface photographs necessary to carry on extensive lunar geological studies, would determine the detailed structure of the lunar gravitational field needed to obtain important information about the interior of the Moon, and would obtain a complete map of the remanent lunar magnetic field as its major objectives.

- The Kepler spacecraft would make an elliptical orbit around Mars for the purpose of exploring the planet's upper atmosphere and its interaction with the solar wind. It would also answer longstanding questions about the magnetic state of Mars.

- Agora, an asteroid mission, would either fly by or rendezvous with several asteroids, depending on the propulsion system that becomes available. The more ambitious asteroid missions, involving detailed, rendezvous-based studies of several mainbelt asteroids, would rely on a cooperative project with the United States.

Among the more ambitious missions that have been discussed, most are primarily suited to major international collaboration, at least so long as the European space science program remains as small as it is at present. 
6

\section{CASE FOR INCREASING LEVEL OF NASA-ESA COOPERATION}

The challenge and the promise of planetary scientific exploration have been analyzed separately by the scientific communities of Europe and the United States. In both instances, these analyses led to the conclusion that planetary investigations promise major scientific advances in understanding and discoveries about the nature of our solar system, including the characteristics of the planets and other objects in the solar system, the forces and processes that govern their behavior and their evolution, the nature of Earth and its environment, the apparently common origin of the Sun and planets, and the physical mechanisms that shape the behavior of large cosmical systems and their relation to more distant objects. As a consequence, scientific communities in both the United States and Europe have recommended that planetary studies be one of the major foci of effort in the programs of space scientific research.

In analyzing the outstanding objectives of planetary research, as described in the previous section of this report, and in planning to accomplish these objectives, the two scientific communities have proposed scientific exploration strategies that have many common or overlapping elements. In some cases, the specific planetary spacecraft missions that are contemplated by the two communities are aimed at similar goals and may have similar or complementary designs. These similarities of purpose and intention are, of course, not accidental but rather reflect concentration on those spacecraft investigations that present themselves as being of the highest scientific importance and as having the greatest exploratory potential. The similarities also reflect the traditionally close intellectual collaboration on scientific investigations that exists between Europe 
and the United States. There is general agreement with respect to the definition of the major scientific questions and objectives that should be addressed by continuing exploration. The similarities in many of the planetary projects contemplated by the U.S. and European scientific communities also reflect the similar levels of technological prowess on the two sides, although until recently it has been the United States, more than Europe, that has chosen to develop and use these technological capabilities for expansion of knowledge about the solar system. Finally, the similarities in contemplated planetary scientific programs reflect the fact that the overall level of economic and other resources in the European community as a whole and in the United States are roughly in balance, leading to the emergence of comparable ambitions.

In planetary exploration the U.S.-European collaboration until now has been incomplete in that, of the two communities, only the United States has had a vigorous and, for a time, sustained program of planetary spacecraft investigations. For the most part, European participation has been at the level of individual scientists or groups of investigators taking part in projects wholly conceived and executed by the United States. This participation in the U.S. planetary spacecraft program has complemented the other planet-oriented experimental and theoretical research in Europe. The active U.S. planetary program, carried out during the two decades from 1960 to 1980 , thus fostered the development of outstanding planetary research groups, not only in the United States, but in Europe as well. European investigators and scientific research teams gained access to planetary objects with the opportunity then to conceive, build, and carry out experiments that otherwise would be beyond their reach. The United States benefited from the expertise, ideas, and in some cases, unique capabilities of European scientists, thus enhancing the quality and economy of its planetary program.

As we approach the mid-1980s, circumstances have changed both in Europe and in the United States. The European community has greatly expanded its independent space operational capabilities. With the development of the Ariane rocket series, Europe has developed the ability to launch a range of significant planetary spacecraft. The European Space Agency (ESA) already has undertaken its first deep-space, planetary-type project-the Giotto mission to Comet Halley. In the United States, major efforts have been devoted to rebuilding planetary exploration by 
constructing a planetary program that contains a stable base of activity, consisting of modestly conceived projects, and that will provide a basis for occasional missions of greater complexity and ambition.

The challenges of continuing solar system investigations are large, and the opportunities for projects are many. Scientific exploration of the solar system constitutes one of the major intellectual and technical endeavors of our time. The foreseeable space science programs of Europe and of the United States will not accomplish all that is contemplated and desired. This conclusion is not altered even upon taking into account the vigorous planetary programs of others-notably the Soviet Union. Since the United States and the nations of western Europe share a common heritage as well as a long tradition of friendship and cooperation, it is appropriate to consider approaches by which a substantial measure of solar system exploration can be carried out collaboratively; the advantages to both sides are manifest, and the possible approaches to collaboration are several.

Altogether there are four levels of cooperation that can be identified between the U.S. National Aeronautics and Space Administration (NASA) and the European Space Agency of successively increasing ambition and significance. All go beyond the presently minimal collaboration that has been restricted, in the planetary program, to individual scientists or groups from one side of the Atlantic having limited participation in missions designed and executed largely by the other.

Nevertheless, while ultimately the Joint Working Group (JWG) shall advocate more intensive levels of cooperation in planetary exploration, it strongly supports the principle that separate agency projects continue to be open to proposals from scientists from the other community. By following this policy, which was pioneered by NASA, the quality of scientific investigations will be enhanced, and a valuable cooperative spirit will be promoted.

\section{LEVELS OF COOPERATION}

The least intensive further level of cooperation involves joint planning of long-term exploration strategies and collaboration in the planning of independently executed mission programs. This would minimize the likelihood of undesirable duplication in and 
provide for the most effective overall attack within the constrained resources that are available.

A second possible level involves specific coordination of independently executed missions to a single target. By coordinating these missions that are indicated by scientific considerations, the two agencies could increase the scope of their scientific investigations in an economic way, while at the same time each agency could preserve its independence in the planning, design, and execution and avoid being dependent on the other partner.

The two types of collaboration just described involve minimal amounts of truly joint activity. They share the attribute that the real impact is small on the programs that either side might carry out alone. Although such limited kinds of cooperation would have a positive influence on any planetary projects that might be carried out, this approach alone would realize only few of the special benefits that can come from close collaboration. In order to further common scientific interests and pursue initiatives that otherwise might not be realized, more significant kinds of collaboration will be required. This more intensive collaboration should involve some measure of joint execution of single projects.

Even here there are at least two levels of collaboration that can usefully be incorporated into the planetary programs. At the most modest level, there are projects for which the major initiative, the design, and the execution of a mission reside with a single space agency, but for which one or more of the major enabling components is provided by the other side. Examples of this approach, which have been used in the past or are under way, include the NASA Galileo mission to Jupiter for which the Federal Republic of Germany is providing the retropropulsion module, and the ESA Solar-Polar mission for which, in the presently reduced configuration, NASA still is providing the launch. In both instances, scientific investigations are being built and carried out by teams from both sides of the Atlantic. In the future, it is expected that similar opportunities will present themselves for this kind of valuable but limited cooperation; potential candidates in this regard could be found in the NASA-Solar System Exploration Committee (SSEC) core program as well as in missions contemplated by ESA alone. In each case, appropriate arrangements for constructing and sharing the payload of scientific instruments can be devised.

The most ambitious, and potentially the most rewarding, approach to collaboration in planetary exploration involves the joint 
planning, design, construction, and execution of major planetary projects. It is with this approach that NASA and ESA can play major enabling roles in bringing about projects that otherwise would not occur in the foreseeable future. At the same time, it should be recognized that this intimate kind of collaboration could involve new relationships between the space agencies and will present new challenges that, however, are commensurate with the advantages that such close collaboration can provide.

\section{COOPERATION IN INDEPENDENT PROGRAMS}

It is the view of this Joint Working Group that such ambitiously conceived collaborative projects should be a goal for the near future, one toward which the two space agencies should begin working now. The eventual goal should be to mount major collaborative missions, jointly planned and executed, for which collaboration is a significant enabling aspect. In a well-planned cooperative program the less intensive approaches to collaboration described above will also be used. Early consideration should be given to instances in which significant elements of collaboration could be implemented by making use of opportunities presented in the NASA-SSEC core planetary program, either between NASA and ESA or one or more European nations individually.

The remainder of this report will analyze several candidate missions that could be the basis for major collaborative projects and will present recommendations for beginning new levels of collaboration in scientific planetary exploration. The JWG's recommendations include a small number of carefully selected missions, which cover a reasonably broad spectrum of science and technology, as well as costs. Recommendations are made both for projects that would fit within the NASA planetary core program and the ESA mandatory science program and for projects that will require special or optional initiatives on both sides.

An important aspect of many of the proposed candidates for jointly conducted missions is that they pose technological as well as scientific challenges. Examples of these challenges include development of an efficient low-thrust propulsion system that could be used on a variety of planetary spacecraft missions, and achievement of advances in robotics necessary for an "intelligent" roving vehicle operating on the surface of another planet. The dedicated 
acceptance of such technological challenges and the visible demonstration of continued technological advances should be essential aspects of the cooperative program of planetary exploration between the United States and Europe. This approach represents a fundamental departure from the principles that have most recently guided planning for future planetary missions in the United States. The NASA-SSEC core program, including the Venus Radar Mapper mission, has had as its hallmark the efficient application of existing technology to conduct missions of sharply limited scope and cost. In contrast, the more ambitious missions proposed as part of a joint European-U.S. effort can permit, and in fact demand, that technological innovation be an integral aspect of mission planning, development, and execution. As such, the more ambitious of these projects could represent an important part of the needed complement to the basic SSEC core program. The development of new technologies, as with the undertaking of new science, should in large part be shared equally between the two partners.

The program proposed here for joint U.S.-European missions of planetary exploration is based firmly on the premise that the separate agency programs of planetary exploration will not diminish in their vigor. The collaborative missions described in the following section do not by themselves substitute for vigorous NASA and ESA programs in planetary science. Nor are the proposed missions to be viewed in competition with the enunciated plans of the separate agencies for planetary missions in the near future. Rather it is hoped that the collaborative projects proposed as candidates for jointly conducted missions, by their greater ambition and prospect for scientific return, will significantly enrich the existing agency programs and will enable the United States and Europe to undertake together projects that neither side would be able to initiate alone during the foreseeable future. 


\section{7 \\ CANDIDATE COOPERATIVE PROGRAMS}

\section{CANDIDATE COOPERATIVE PROGRAMS}

The Joint Working Group (JWG) and its study teams have identified several areas of the planetary exploration that lend themselves well to a cooperative approach by Europe and the United States. Furthermore, it has identified particular missions that by virtue of their broad significance, the degree to which they would complement other parts of existing planetary programs, their suitability for joint implementation, and their technological challenge are especially attractive as new U.S.-European initiatives.

The Joint Working Group's three study teams have reported an impressive set of candidate projects for consideration as joint endeavors. Without this critical foundation, it would have been impossible to construct useful recommendations, and the Joint Working Group is grateful for the contributions of the many scientists and engineers, and especially the three chairpersons, who devoted their time and effort to preparing the study team reports.

The study teams were given specific requirements into which they were to fit their proposed missions. The requirements were based on the Joint Working Group's overall charge, which is given in Chapter 2 of this report. Moreover, the study teams were given specific cost guidelines within which to work. These cost guidelines were based on the JWG's desire to put forth recommendations for 
high-quality cooperative initiatives that could lead to new projects in several foreseeable circumstances. The cost estimates used by this Joint Working Group, and contained in the appendixes, were prepared in consultation with both the National Aeronautics and Space Administration (NASA) and the European Space Agency (ESA) and are intended only to give a general picture of the relative costs and programmatic impacts of the various proposals; they do not represent firm opinions about final costs.

The study teams generally were successful in constructing candidate cooperative missions. It is important to realize that the requirement that missions be equally divided in costs and responsibilities can, in any case, be regarded only as an approximation. Flexibility is needed in dividing cooperative projects so that logic and expedience take precedence over rigidity.

At the low end, the cost guidelines given to the study teams include missions that can fit within the base level of mission activities of both NASA and ESA. These are missions with a total cost in the range 400 to 500 million (dollars or accounting units), excluding the costs of launch and mission operations, so that each side's participation can fit within the boundaries of missions presently acceptable in the core or mandatory science programs of NASA and ESA. In some cases, these projects already are being discussed by ESA and NASA for possible joint implementation, a development that is welcomed and encouraged.

The largest advantages-both scientifically and technologically-will generally come from larger projects that require new levels of initiative by the two space agencies. In ESA these projects could involve interested countries through optional programs; in NASA these projects could involve augmentations to the presently conceived core planetary program. The JWG strongly recommends that such larger cooperative missions be started at an early time.

In defining which missions would be included for consideration among the larger recommended initiatives, the Joint Working Group was presented with many possibilities. It holds the view that planetary exploration should be expanded significantly beyond the presently minimal level of activity but also recommends that such expansion take place in a measured and sustainable way.

The JWG's recommended cooperative program aims therefore not only at particular immediate objectives but also at developing 
advanced technological capabilities and laying the scientific foundation for continued and more advanced planetary exploration in the future.

The advantages to be gained by a long and continuing sequence of cooperative planetary explorations are many. However, there is a long lead time between the start of a project, its implementation and completion, and its follow-up. There is little to be gained by postponing the start of significant cooperation, and much to be lost. The JWG urges, therefore, that cooperation in planetary exploration be begun quickly and vigorously.

\section{STUDY TEAM REPORTS}

Although each of the three study team reports was fully responsive to the charge from the Joint Working Group, there were differences in the manner and form of the reports and differences in the programmatic priorities ultimately accorded the various missions proposed.

\section{Inner Planets}

The Inner Planets Study Team reported three candidate missions to the JWG: the Mars Dual Orbiter, the Mars Surface Network, and the Mars Rover.

The Mars Dual Orbiter mission is based on two independent mission proposals that are being considered separately with NASA and ESA. These are the NASA Mars Geoscience and Climatology Orbiter (MGCO) and the ESA Kepler Mars Aeronomy Orbiter mission. The study team makes a case that by flying both of these missions in a closely coordinated way, involving their simultaneous presence around Mars for a significant period of time, enhancements of the overall science return can be achieved. The JWG accepts these arguments and believes that should both missions be approved by their respective agencies and should it be programmatically feasible to fly them in a coordinated way, then every effort should be made to do so.

For the most part, however, these missions remain a pair of single-agency projects. As such, they will be assessed mainly on that basis and, therefore, lie outside the further purview of this 
Joint Working Group, which is specifically reporting recommendations for projects that are inherently cooperative in their design and implementation.

The Mars Surface Network addresses large and important questions of Mars science. A network of scientific instruments, carefully arrayed over the surface of Mars, offers the opportunity to mount unique investigations of the planet's global characteristics. Technically, the project divides well into two parts that integrate to yield a high-quality scientific mission. The devices that would be deployed in a surface network could be built by one side, while the orbiter, Earth-communications link, and perhaps orbital-science bus could be built by the other side. Significant technical challenges could be met in both parts of the project. In terms of the size and cost, a joint Mars Surface Network mission could be fit within the current ESA and NASA mandatory and core programs.

Some uncertainty should be recognized. A major scientific objective of such a network mission would be seismological studies of the interior structure of Mars. At present, the seismicity of the planet remains unknown. The limits on seismic activity of Mars derived from the Viking Lander experiment are not meaningful because of that instrument's poor sensitivity and loose coupling to the solid part of the planet. However, the lack of any reliable global seismic event must be recognized. It is important that a Mars network be arrayed with close attention to a proper mix of long and short baselines, so as to ensure optimal retrieval of the available data.

A network of small scientific packages would offer a significant instrumentation challenge. The design of the device itself needs to offer sufficiently long-lived and reliable operation to ensure completion of the various experiments. Moreover, considerable effort must be expended to ensure the development of needed analytical instruments. This situation is not unique; commitment to a program of space science requires equal commitment to the development of new scientific instruments and techniques. Indeed, a large part of the reward to society that comes from space exploration is derived from the advances in technology that come from meeting scientific challenges. More on the subject of instrument development is covered in Chapter 8.

The Mars Rover mission offers another approach to these as well as other science objectives by providing the capability for 
more intensive and complete examination of the planet over several extended regions. This mission has attractive features that are commensurate with its ambition and challenge. Development of such a roving vehicle would open the way to a continuing sequence of advanced explorations of Mars. The detailed investigations needed to decipher surface evidence of the complicated environmental history of Mars could be carried out, after the development of the needed analytical instruments. In the future a roving vehicle could offer an excellent approach to gathering an intelligently selected and diverse sample of Mars matter to be returned to Earth for analysis. This ambitious and critically important sample return is a major component in the continuing investigation of the solar system.

Technologically, the roving vehicle presents an attractive challenge in that the design, construction, and remote operation of such a device require advances in the application of robotics and remote manipulation. In terms of the size and cost of the project, a Mars Rover mission would require augmentation to the present NASA and ESA science programs. The project could be divided between the two sides in several different ways. One possibility would be to have one side build the vehicle itself while the other side built the spacecraft and delivery system. Both parts represent technological challenges in which the resultant capabilities could have significant later applications. On the other hand, it is also conceivable that both agencies would want to take part in the technologically exciting task of designing and building the surface rover. The JWG believes it is appropriate to leave these detailed questions to later negotiations between the responsible agencies.

\section{Outer Planets}

The Outer Planets Study Team reported a single candidate mission to the Joint Working Group, a Saturn Orbiter and Titan Probe mission. The study team recommends that this project be planned to include a newly designed atmospheric entry probe to be sent into Titan's atmosphere and a Saturn orbiter of the Galileo class. The study team makes a case that the major science objectives that ought to be accomplished in the next phase of Saturn system studies are not likely to be met with a spacecraft of substantially reduced capability. The JWG generally accepts this 
analysis but notes that the question should be fully resolved by further agency studies.

\section{The Primitive Bodies-Asteroids and Comets}

The Primitive Bodies Study Team has presented the JWG with a relatively long list of cooperative mission candidates, with considerable overlap in the designs and objectives, so that they are not all independent possibilities. The study team's list includes three varieties of asteroid orbiter missions, two kinds of sample returns, and a comet rendezvous.

The comet rendezvous mission with a chemical rocket, as proposed by the study team, is essentially similar to the independent comet rendezvous mission being considered by NASA as the first initiative in its new Mariner Mark II series. There are significant opportunities for limited elements of international cooperation in that mission. As noted elsewhere, there are discussions under way between NASA and the Federal Republic of Germany. On the other hand, this project, by virtue of its present size and design, does not fit into the class of projects that are suitable as major collaborations between NASA and ESA, and on which the JWG is concentrating its attention.

Beyond the firmly planned or anticipated missions to the primitive bodies, which include the 1986 Comet Halley flybys and the NASA Comet Rendezvous mission, the next major steps in primitive bodies exploration are a multiple asteroid orbiter mission and a comet nucleus sample return. Planning and design of a reliable device to achieve a comet nucleus sample return are likely to require substantially more complete knowledge of the character of comet nuclei than is currently available and more than will be attained through rapid comet flyby investigations. Thus, it seems prudent to wait to start such a project until after the prerequisite information has been gathered by a comet rendezvous mission. 


\section{8 SPECIFIC RECOMMENDATIONS}

In order to formulate its recommended program of planetary missions to be carried out as cooperative ventures by Europe and the United States together, the Joint Working Group (JWG) took into account the present state of planetary knowledge produced by earlier studies and missions, the highest priority scientific objectives that lie before us, the planetary projects presently under way within the two separate space agencies, the projects that are expected to be carried out in the near future or that are under consideration, and the states of technological readiness and timeliness of the candidate cooperative missions. The program that is put forward builds on the knowledge gained through earlier studies, takes advantage of and expands the expertise of the relevant scientific communities, exploits technological interests, and challenges and advances the technological capabilities of the participating agencies. Finally, the proposed program complements the existing projects and plans of the separate agencies by enabling missions that are beyond the scope and ambition of projects anticipated by either agency alone.

\section{EXISTING PROGRAMS}

Several of the past projects taken into account in this planning are still under way and will continue to contribute important planetary knowledge during the remaining years of their extended mission times. These include the Voyager flybys of Uranus and Neptune, which will accomplish the first reconnaissance of those planets, and the Pioneer Venus Orbiter mission, which continues to 
return significant data from studies of Venus' upper atmosphere and its interaction with the solar wind. Several of the projects taken into account are approved and in various stages of construction; these include the ESA Giotto mission to Comet Halley, which will carry out the first close flyby investigations of a comet, the NASA Galileo Jupiter Orbiter and Probe, which will carry out intensive studies of the Jovian system, and the NASA Venus Radar Mapper, which is planned to carry out detailed geological investigations of the hidden surface of Venus.

Also taken into account in the Joint Working Group's deliberations were the major projects and plans of other nations, insofar as they were known. These include the Soviet Union's VEGA mission, consisting of flyby studies of Comet Halley as well as probe investigations of Venus. Note was also taken of the continuing vigorous and intensive series of Venus investigations under way by the Soviet Union, the most recent being the two-spacecraft radar mapping mission launched toward Venus in the spring of 1983, which began returning data in late 1983 .

In addition to these ongoing projects, both the European Space Agency (ESA) and the National Aeronautics and Space Administration (NASA) have several independent projects that are being considered for possible implementation in the near future. In the United States several missions in the Solar System Exploration Committee's (SSEC's) core program are anticipated for initiation in the late 1980s. From the SSEC core program the JWG has specifically taken into account the Mars Geoscience and Climatology Orbiter, and the Comet Rendezvous with possible asteroid flybys. The Mars orbiter will carry out a global geochemical survey of the planet in order to gather information about the character and evolution of its surface and will collect information about the planet's climate cycles, complementing the earlier studies of Mars carried out by the Viking project and its predecessors. Moreover, this mission will provide a stepping stone to future intensive investigations of Mars.

The Comet Rendezvous mission will provide the first sustained closeup studies of a comet, enabling detailed investigations of the nucleus, coma, and tail, while following the comet during a substantial part of its inner solar system traverse. This mission is a necessary next step in a program of comet science; it is the natural successor, in this discipline, to the European Giotto and the Soviet VEGA mission to Comet Halley. The Comet Rendezvous will also 
build the foundation of knowledge needed to plan for an eventual comet sample return.

In the ESA program, the JWG has taken account of the potential Kepler project, a Mars-orbiting spacecraft aimed at investigations of the Martian upper atmosphere and its interaction with the solar wind. The implementation of this project, which presently is under consideration for inclusion in the ESA program, would provide information to fill some of the important gaps in the understanding of Mars.

The JWG takes particular note of the pair of potential Marsorbiting spacecraft-the NASA Geoscience and Climatology Orbiter and the ESA Kepler mission. These are planned as independent missions, each of the pair possibly to be carried out by one of the agencies alone; however, should both be carried out on schedules that permit simultaneous operation about Mars, then coordination of the investigations could provide significant benefits to the two programs, enhancing the cumulative advances in knowledge about that planet. Areas in which coordinated, simultaneous measurements could lead to significant and unique scientific advances are enumerated in Appendix A. Of course, optimum use of these two Mars science opportunities would be gained by drawing on the resources of the U.S. and European scientific communities for both spacecraft.

The cooperative mission program proposed by this Joint Working Group is designed to complement those existing and contemplated programs and to strike out toward scientific and exploratory objectives of somewhat greater ambition, but which still are accessible to modest extensions of the current programs. The proposed cooperative program puts forward three projects, which have been designed so as to be susceptible to joint planning, construction, and execution during approximately the next 15 years. Each of the projects satisfies the criterion that it is susceptible to a roughly equal division of responsibility and costs, as well as technological and scientific challenges.

The proposed missions span the three major regimes of the solar system: the inner solar system, the outer solar system, and the primitive bodies-asteroids and comets. This study recognizes the fundamental advances that remain to be made in each of the three major areas of planetary science and reflects the importance of a balanced advance in knowledge across the frontier of this research. 


\section{THE INNER SOLAR SYSTEM}

For the inner solar system the Joint Working Group's recommended mission focuses on intensive studies of Mars. The sequence of earlier investigations, carried out by the United States, and culminating with the Viking Project in 1976, left a legacy of important and unresolved questions that are critical to understanding that planet and the terrestrial planets as a class, including Earth.

The other planetary bodies in the inner solar system-Venus, Mercury, and the Moon-are not included in these recommendations for several reasons. Venus is the object of continuing intensive and successful study by the Soviet Union. These studies, together with the United States' recent Pioneer Venus Orbiter and Probe mission and the Venus Radar Mapper mission, are advancing our understanding of Venus at a steady pace. The JWG believes that, in the overall context of the present planetary programs, additional resources at this time would best be used if directed elsewhere.

Mercury poses significant scientific and technological challenges that continue to go unmet. The JWG has not included Mercury missions in the present plan for several reasons, the primary one being based on the Committee on Planetary and Lunar Exploration (COMPLEX) recommendation that inner solar system science investigations should focus on the triad of similar atmosphere-bearing planets-Venus, Earth, and Mars.

Earth's Moon, despite its importance, is not included in these plans because, for scientific investigations in the near future, it is primarily suitable for small-scale investigations more readily carried out by a single space agency. However, some of the geophysical and geochemical investigations targeted to Mars could very profitably and economically be reproduced for application to the Moon.

\section{THE OUTER SOLAR SYSTEM}

With the use of currently contemplated propulsion technologies, spacecraft travel times to the outer solar system planetsJupiter, Saturn, Uranus, Neptune, and Pluto-are relatively long. Because of this, missions to these planets involve long project lifetimes and operations, with the associated higher costs, and involve long hiatuses between the launch and the return of any primary data. On this basis the Joint Working Group ruled the farthest 
of these planets out of consideration as targets of an early joint project. The next stage of investigations of the Jupiter system is already under way with the NASA Galileo project.

The Saturn system has the highest priority among outer solar system scientific investigations after Jupiter. Saturn system missions have been introduced as potential candidates for the early 1990s in both the U.S. and the European programs. According to project analysts, significant economies can be realized by making optimum use of technical heritage from the Galileo mission. Because of the scientific importance of the Saturn system and the timeliness of such a project, the Joint Working Group has included a Saturn-system mission in its recommendation, despite the long trip time.

\section{THE PRIMITIVE BODIES}

With the relatively ambitious flybys of Comet Halley planned by Europe and the Soviet Union for 1986, and the substantially less ambitious comet flybys planned by Japan and the United States, the initial reconnaissance of comets will have been accomplished by the middle 1980s. Comets and asteroids-because they are thought to be the most primitive of large solar system objects, carrying unparalleled clues about solar system formation and evolution processes-occupy a unique position in our thinking about solar system exploration.

The Joint Working Group's recommendation includes a mission to several asteroids. For comets, the next major step beyond the 1986 flyby studies of Comet Halley will be taken by a comet rendezvous planned to occur in the NASA Solar System Exploration Committee's core program, possibly with some level of participation by Germany. After the comet rendezvous, the return to Earth for intensive laboratory analysis of a high-integrity sample of comet nucleus material will be the highest priority scientific objective of continuing cometary investigations. The earlier rendezvous investigations are important to the technical planning for sample acquisition. The investigation of asteroids must take account of the fact that some samples of these bodies are believed to be already in terrestrial laboratories from meteorite falls. Yet the structures of these bodies and the evolutionary processes to which they have been subjected remain to be determined. Moreover, the extent to which meteorites are representative of the general 
class of asteroidal objects needs to be established. A major advance at this frontier of knowledge will require detailed study of a representative set of at least five or six of the main-belt asteroids.

\section{RECOMMENDED JOINT PROJECTS}

The JWG recommends three cooperative planetary projects for joint implementation by the European Space Agency and the National Aeronautics and Space Administration. Listed in order of the recommended launch sequence, without implied scientific priority, they are

- the Titan Probe and Saturn Orbiter mission,

- the Multiple Asteroid Orbiter with Solar-Electric Propulsion, and

- the Mars Surface Rover.

Each of these missions is of the highest scientific importance. The JWG recommends that all three be carried out on a schedule that will have them completed by about the end of the century. The characteristics of each are summarized below.

\section{Titan Probe and Saturn Orbiter}

This mission to the Saturn system consists of two components launched together. The main spacecraft would carry an atmospheric probe, which it would release prior to arrival at Saturn. The probe would enter Titan's atmosphere and carry out scientific measurements from the upper atmosphere to the impact at Titan's surface. Titan, the largest moon of Saturn, is of special interest, in part, because its atmosphere is thought to be similar in important respects to the prebiotic atmosphere of Earth, in which nonbiogenic chemicals first became organized into living systems.

After serving as the radio communications relay link for the Titan entry probe, the main spacecraft will go into orbit around Saturn itself. Executing some dozen orbits of the planet, each time passing again by Titan, the spacecraft will carry out investigations of Saturn, its ring system, and its magnetosphere as well as Titanincluding radar observations of its surface.

The major scientific objectives that would be addressed by the mission are comparable in scope to objectives targeted in 
the NASA Galileo-Jupiter mission and include the thermal structure and composition of Saturn, its atmospheric dynamics, the character of its magnetic field and magnetosphere, the dynamical behavior of its ring and satellite system, the nature of Titan and the composition and evolution of its atmosphere, the states of prebiotic molecules and their interactions, and the behavior of icy satellites.

\section{Multiple Asteroid Orbiter with Solar-Electric Propulsion}

The asteroids are largely confined to a belt lying between the orbits of Mars and Jupiter. Too small to have undergone major planetary evolution processes, they retain evidence of the processes by which they were formed along with the planets some 4.5 billion years ago. Systematic variations, in remotely detected properties, from the inner to the outer parts of the asteroid belt, apparently reflect variations in the radial properties of the disk from which the planets formed.

The Multiple Asteroid Orbiter mission will use a technically ambitious solar-electric propulsion system to accomplish orbitalmode investigations of five or six main-belt asteroids. In this mode the spacecraft goes into temporary orbit about an asteroid for a period of the order of a month and carries out detailed investigations during that time. Upon completion of the study, it moves on to another selected asteroid. The duration of a mission is some 5 to 7 years, and during each traverse, from one asteroid rendezvous to the next, the spacecraft can be caused to fly near one or two additional asteroids in order to carry out a short-duration, cursory examination.

The scientific objectives of the Asteroid Orbiter mission include determining the chemical and mineralogical compositions and the physical structure and heterogeneity of each object in order to investigate the chronology of processes of formation and evolution. These investigations are critical to the growth of knowledge about solar system formation processes. In addition, studies of the asteroids' space environment will include possible emission of dust and gas and possible remnant magnetic fields. 


\section{Mars Rover Mission}

In many respects the most ambitious of the JWG's recommended projects, the Mars Surface Rover mission would consist of two or three mobile vehicles landed on the Martian surface at selected sites. The vehicles would go to Mars on a single ShuttleCentaur launch. Each vehicle, carrying about 100 kilograms of scientific instruments, would have the capability of moving-under direction from Earth-one to several kilometers in a day, so that, over the course of a year to two, each would cover some 1,000 kilometers. From time to time in its traverse, the rover would stop to make intensive local measurements and perhaps to plant an instrument package for seismic and other network investigations.

The scientific objectives include the investigation of the structure, composition, and context of Martian rocks, soils, and volatile matter for the purpose of determining the history of the Mars environment and its climate cycles, and the processes that have produced its diverse surface features. In addition, the project would carry out studies of the planet's interior activity and structure, as well as its magnetic field; the rover would explore the present inventory of volatiles, including subsurface water ice.

The Mars Surface Rover mission is the least well defined of the JWG's recommendations. While the basic concept is sufficiently well understood to be judged sound, there remains considerable latitude in the precise design of the vehicle and the landing system and in the construction of scientific instruments that are suitably ambitious and incisive in their measurements to take full advantage of research opportunities opened by such a mission. While these technological challenges point to the need for significant amounts of preparation in all of these areas, the JWG believes that such challenges are appropriate to the planetary programs and beneficial to the societies that take them up.

\section{IMPLICATIONS FOR THE PROGRAMS}

In analyzing the cooperative missions, the JWG has adhered to the principle that an equal division of responsibilities between NASA and ESA should be sought for each project. The JWG believes that, over time, such an equal division should be a goal but does not recommend a rigid insistence on such exact division of each individual project. For each project, the actual division 
should reflect structural, technical, and management realities. It is obvious that truly cooperative projects can sustain substantial imbalances in the division of responsibilities and costs while still reaping enormous benefits for both sides.

Two of the JWG's recommended missions can be fitted within the mandatory or core programs of ESA and NASA. These are the Titan Probe and Saturn Orbiter mission and the Multiple Asteroid Orbiter with Solar-Electric Propulsion. In addition, both of these missions have the further attribute that discussions are already taking place between NASA and ESA to explore the possibility of mounting them as joint projects. The Titan-Saturn mission is technically ready to implement. The solar-electric propulsion for the asteroid mission needs additional development and construction time, but considerable interest already exists-in Europe and particularly in West Germany-in mounting a development project leading to a substantial operational system for use in the early 1990s. The JWG recommends staggered early starts on both the Titan-Saturn mission and the Multiple Asteroid Orbiter mission, leading to a launch of the former in approximately 1992 and a launch of the latter in 1994.

It is noted that these launch dates are constrained entirely by fiscal considerations in the present agency programs. Scientific and technical considerations do not dictate such a slow pace and, indeed, would indicate that it would be advantageous if they were carried out earlier. The JWG would urge a faster implementation but notes that this requires political decisions to increase the pace of planetary science beyond its presently low level.

The Mars Rover mission does not fit into the core and mandatory programs as they presently exist. Initiation of this mission, particularly from the European perspective, will require either an expansion of the mandatory science programs or the undertaking of this mission as a special project, with the main participation coming from those nations with especially keen interests in pursuing vigorous programs of planetary science and technology. Technologically, the Mars Surface Rover would benefit from substantial advanced development effort. This should be aimed both at the technology of constructing and operating a robot vehicle and at the development of scientific instruments designed to capitalize on the operational capabilities of such a vehicle. The JWG recommends that the Mars Surface Rover mission be implemented on a schedule that leads to a launch around 1996. 


\section{TECHNOLOGY ISSUES}

Several important factors weighed heavily in the Joint Working Group's formulation of this recommended cooperative program of planetary exploration. In addition to considerations of scientific importance and technological timeliness, discussed earlier, the JWG weighed the extent to which various projects incorporate technological advances that could lead to more ambitious scientific endeavors. In this regard, the Mars Rover mission and the Multiple Asteroid Orbiter with solar-electric propulsion both stand out.

\section{Solar-Electric Propulsion}

The development of a low-thrust solar-electric propulsion system has been discussed for some time, both in the United States and Europe. The advantages offered by such a system are well established with respect to the payload carrying capacity and the flexibility in launch and mission planning. The state of the basic technology in this area is such that scientists are ready to proceed to the design and construction of an operational system. Beyond the Multiple Asteroid Orbiter mission, solar-electric propulsion would be applicable to a comet nucleus sample return-one of the major goals in the continuing investigation of primitive solar system objects. Within the inner solar system, solar-electric propulsion would provide the launch capability for a range of ambitious projects in the future. Furthermore, low-thrust electric propulsion has potentially broad applications for operations in Earth orbital space.

\section{Robotic Planetary Rover Technology}

Semi-autonomous remote operation of a scientifically equipped roving vehicle on the surface of another planet is a challenge to scientific capabilities in robotics, automation, and remote manipulation. Meeting this exciting challenge could be expected to provoke a significant advance of the overall capabilities in this area, with important contributions going also to society.

Operation of such a vehicle on the surface of Mars, with its astonishingly varied terrains, which indicate large climatic environmental variations in its history, would produce major advances 
in scientific knowledge of that planet. Moreover, such a mobile capability for Mars could provide the initial basis for a long-term scientific presence on that planet and it could greatly enhance the ability to gather a set of Mars samples chosen to answer the most important questions currently asked about that planet.

\section{Scientific Instrument Development}

Both the Mars Surface Rover mission and the Multiple Asteroid Orbiter will carry scientific planetary investigations into new modes, which will bring expanded capability for intensive measurements on these bodies over extended periods of time. In both cases these new modes of exploration provide platforms from which innovative approaches to analysis could be carried out. The JWG strongly recommends that programs of advanced development of scientific instruments for use on these missions be begun at the earliest time both in Europe and in the United States. 


\section{APPENDIXES: WORKING PAPERS OF THE STUDY TEAMS}

PRECEDING PAGE BLANKK NOT FILMED 


\section{APPENDIX A: INNER PLANET MISSIONS}

Terrestrial Planets Study Team

Frederic W. Taylor (Chair), Clarendon Laboratory Arden Albee, Jet Propulsion Laboratory G. Balmino, Groupe de Recherche de Geodesie Spatiale Marino Dobrovolny, Istituto Fisica Spazio Fraser Fanale, University of Hawaii Costanzo Federico, Istituto di Astrofisica Spaziale J. E. Guest, University College Harold Masursky, U.S. Geological Survey Andrew F. Nagy, University of Michigan Carle Pieters, Brown University David Stevenson, California Institute of Technology Peter Stone, Massachusetts Institute of Technology U. von Zahn, Universität Bonn

H. Wanke, Max-Plank-Institut für Chemie 


\section{CONTENTS}

PREFACE

SUMMARY 54

INTRODUCTION

RATIONALE FOR THE EXPLORATION OF MARS 57

PRIME MISSION SET $\quad 59$

COST SUMMARY 83

OPTIONS FOR ESA-NASA DIVISION OF

RESPONSIBILITIES 85

RECOMMENDATIONS AND TIMESCALES 86 


\section{PREFACE}

The Terrestrial Planet Study Group (TPSG) was formed in October 1982 by the Joint Working Group (JWG) on Planetary Exploration of the U.S. National Academy of Sciences/National Research Council (NAS/NRC) and the European Space Agency (ESA). Its charge was to examine the various opportunities for space missions to the planetary bodies of the inner solar system and to recommend those that offered the best combination of scientific merit, suitability for international collaboration, and value for money. Cost categories of less than $400 \mathrm{M}(1 \mathrm{M}=1$ million European accounting units or approximately 1 million U.S. dollars), 400 to $600 \mathrm{M}$, and 600 to $1000 \mathrm{M}$ were to be addressed. The group met three times, twice at the Jet Propulsion Laboratory (JPL) in Pasadena and once at the European Space Technology Center (ESTEC) in Holland. The membership of the group was F. W. Taylor (Chair), A. Albee, F. Fanale, H. Masursky, A. Nagy, C. Pieters, D. Stevenson, U. von Zahn, J. Guest, M. Dobrovolny, G. Balmino, and C. Federico. P. Stone and H. Wanke were nominal members but attended no meetings. J. Blamont and K. Runcorn of the JWG also participated actively in the work of the TPSG. Technical support was provided by the National Aeronautics and Space Administration (NASA) (through J. Beckman and K. Nock of JPL and S. Syges of NASA Ames Research Center (ARC)) and ESA (through G. Haskell of the European Space Agency (ESA) Headquarters and G. Whitcomb of ESTEC), and administrative assistance by European Science Foundation (ESF) (through K. Saul) and NAS/NRC (through D. Kastel). 


\section{SUMMARY}

The Terrestrial Planet Study Group surveyed the opportunities for joint missions and concluded that a large number of excellent possibilities of high scientific merit existed for Mercury, Venus, the Moon and Mars, over a wide range of cost and timescales. Three missions to Mars were chosen as offering the best opportunity for joint European Space Agency-National Aeronautics and Space Administration (ESA-NASA) new starts: Mars Dual Orbiter (cost approximately $300 \mathrm{M}$, where $1 \mathrm{M}=1$ million European accounting units or 1 million U.S. dollars), Mars Surface Network (500M), and Mars Rover (1,200M).

The first of these could be implemented immediately, for data return in the early 1990 s, but it will require extra funds or some other special action by Europe if the opportunity is not to be lost. The other two could follow consecutively, in which case an exceptionally exciting and powerful three-mission program of Martian exploration would result. Alternatively, in a more limited fiscal environment, an extremely strong scientific case could be made for implementing one or the other as an individual project. We recommend that Europe look to implementing its half of the dual orbiter in time to take advantage of U.S. plans for the Mars Geoscience and Climatology Orbiter (MGCO). At the same time, joint preproject studies of the Surface Network and Rover missions should be initiated. One particularly important study that is required is the feasibility of a semihard (as opposed to soft) landing technology for the Mars rover. It is thought that this approach could reduce the total cost of the third mission in the above list to well below $\$ 1$ billion (or 1 billion accounting units). 


\section{INTRODUCTION}

The principal goal of the exploration of the inner planets is to understand the nature and the evolution of the interiors, the surfaces, and where present, the atmospheres of Mercury, Venus, Earth, the Moon and Mars. Consistent with the conviction that a better understanding can be achieved through a comparative study, a balanced exploration program is needed. It is apparent that the largest gaps in our data base at present lie in the areas of the geology of Venus and the climate of Mars. Projects in the United States and the Soviet Union are currently under way to obtain a global reconnaissance of the surface structural features of Venus. Previous exploration of Mars, on the other hand, has produced remarkable and exciting coverage of various geological and atmospheric phenomena on the planet, at the same time leaving relatively unexplored several fundamental fields of Martian science. For example, the existence of a Martian intrinsic magnetic field is still controversial, and no quantitative data via gravitational and mineralogical mapping have been obtained on the internal structure and layering of the red planet's surface. The global atmospheric dynamics, distribution and transport of volatiles, and the structure and photochemistry of the upper atmosphere also need to be quantitatively investigated.

Other poorly known quantities are the magnetosphere of Mars and the interaction of the solar wind with the planet. The characteristics of these interactions are probably alternating between those of Venus and those of the Earth, and from this point of view, Mars is probably a unique example in the solar system.

As a result of considerations such as these, which are detailed further in the following section, and a general belief that Mars, of all the extraterrestrial planetary objects in the solar system, commands the widest interest among scientists on both sides of the Atlantic, the JWG decided at an early stage to focus the attention of the Terrestrial Planets Study Group (TPSG) on mission opportunities for the red planet. In addition to the scientific priority, it was noted that missions to Mars are also attractive for present purposes because the budgets and timescales are far more reasonable, for a given mission complexity, than for most 
other opportunities of comparable scientific interest elsewhere in the solar system. During its initial survey of goals for the terrestrial planets, the TPSG noted the following possibilities as being potentially important in any future studies of joint missions that follow up on the present activity:

- Lunar orbiter,

- Venus orbiter with large probe, and

- Mercury multiple flyby.

Papers were prepared on the scientific rationale for these opportunities and are appended to the present report. Time and resource limitations and the priority given to Mars mean that none has been studied in depth. 


\section{RATIONALE FOR THE EXPLORATION OF MARS}

The study of Mars should lead us toward major new insights into the processes by which planets formed; the effects of initial temperatures, pressures, and compositions on the subsequent internal evolution of the planet; the time history of internal activity that led to the evolution of its current internal structure, surface features, and the atmosphere; the degree to which impact of meteoritic or asteroidal material may have determined planetary composition and crustal structure; the interaction of solar and galactic radiation with the atmosphere and surface materials of the planet and their role in determining atmospheric evolution; the history and dynamics of the atmosphere and hydrosphere and their relationship with the surface and internal processes of the planet; the nature of the environments in which organic evolution can be sustained; and the resulting biological evolution of the Martian surface. In this endeavor we will learn by direct study of the planet and its materials, by comparison to the Earth, Moon, and other planets, and by laboratory studies that tie together observation and theory.

The proposed missions to Mars should address the following objectives (no prioritization is implied by the order):

- Characterize the internal structure and dynamics of the planet.

- Characterize the chemistry and mineralogy of surface and near-surface materials on a global and regional scale.

- Characterize the general circulation, photochemistry, and aeronomy of the atmosphere.

- Determine the chemistry and mineralogy of rock and soil samples, especially as related to the reactive surface chemistry observed by Viking.

- Document geologic characteristics of major landforms, toward an understanding of current and past surface processes.

- Determine the distribution and state of $\mathrm{H}_{2} \mathrm{O}$ and other volatiles toward an understanding of the chemical evolution of the regolith and atmosphere. 
- Measure the planetary magnetic field, and study the interaction of the upper atmosphere with the magnetic field, solar radiation, and solar wind. 


\section{PRIME MISSION SET}

Having decided to concentrate on Mars, the TPSG approached its task by defining a "prime mission set" which contained all of the possibilities thought to meet the following criteria:

- high scientific value,

- technically practical by the end of the century,

- suitable for European Space Agency-National Aeronautics and Space Administration (ESA-NASA) joint implementation, and

- good value for money in three broad cost categories.

Of the complete set (Table A.1), three were selected for further study as best meeting the criteria laid down by the Joint Working Group (JWG) in its instructions to the TPSG (Table A.2). A discussion of each one follows.

\section{Mars Dual Orbiter}

\section{Mission Description}

The Dual Orbiter mission would consist of a close $(\sim 350 \mathrm{~km}$ altitude), circular, three-axis stabilized orbiter plus an eccentric $(\sim 150 \mathrm{~km}$ to perhaps $10,000 \mathrm{~km})$ spinning orbiter, both in nearpolar orbits (Figure A.1). Periapsis for the spinning orbiter would precess completely about the planet in less than one Mars year.

The resemblance of the two components to NASA's Mars Geoscience and Climatology Orbiter (MGCO) and ESA's Kepler missions is not, of course, a coincidence; numerous advantages accrue from combining the missions politically and scientifically, even if they are built and launched separately and arrive at the planet at different times. Some overlap should still be possible if MGCO launches in the 1990 and Kepler in the 1992 windows. More details of the mission parameters may be found in the documentation relating to the individual missions. 
TABLE A.1 Terrestrial Planets Study Group Prime Mission Set

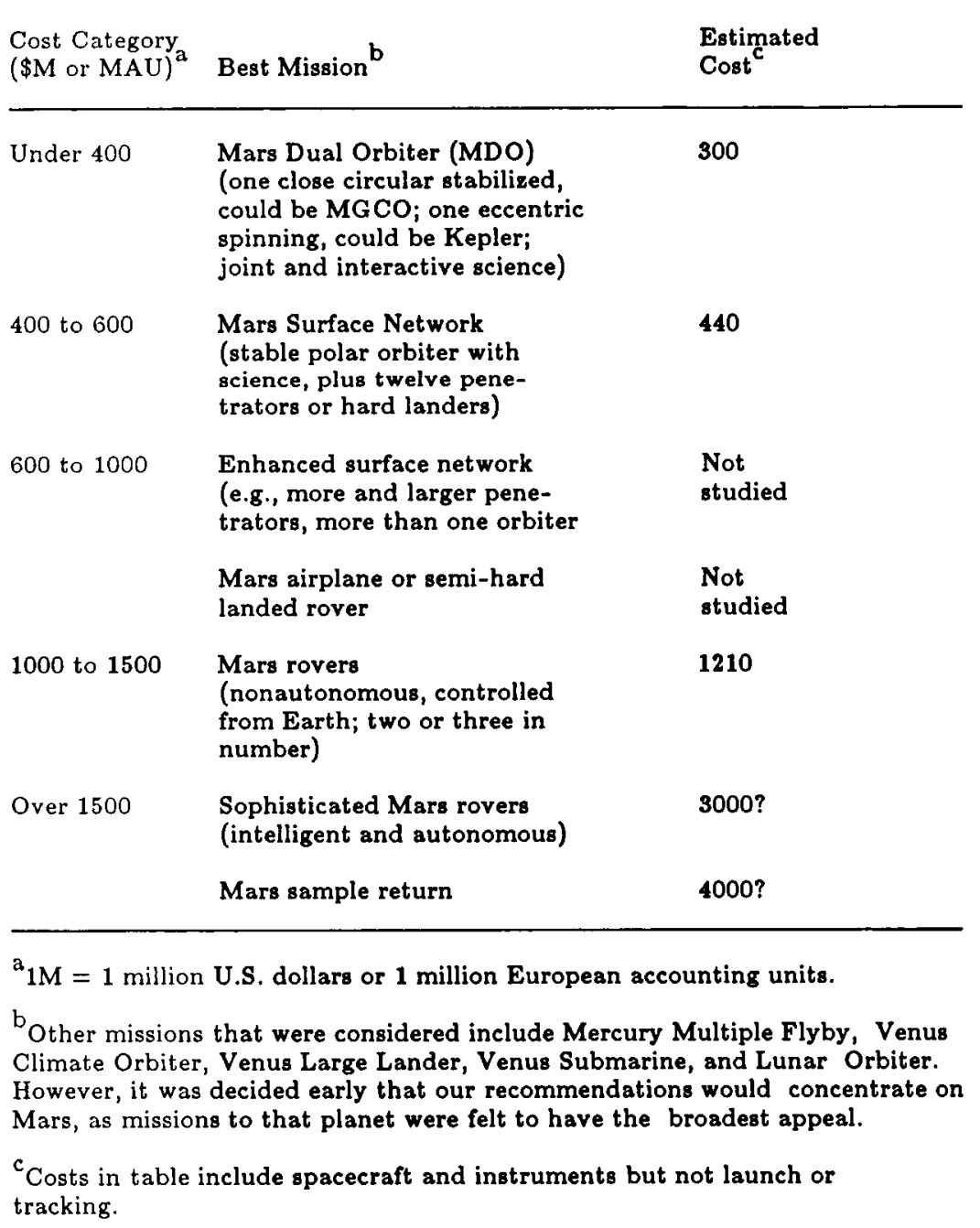

\section{Scientific Objectives}

Although significant advances have been made in the last two decades, major unknowns concerning the properties of Mars and its environment still persist. The scientific objectives for near-future exploration of Mars include the following: 
1. In view of recent space agency actions, especially the selection of the Venus Radar Mapper (VRM) for a new start by NASA, the Terrestrial Planets Study Team should henceforth concentrate its efforts on candidate joint missions to the planet Mars.

2. A possible continuing program with several mission elements, with varied levels of ambition, costs, and complexity should be studied.

3. The JWG endorses studies of

(a) a dual orbiter mission costing $\$ 400 \mathrm{M}$. This could consist of a geochemical/climatological orbiter in a circular orbit and a high-eccentricity orbiter emphasizing aeronomy, along with particle and fields science;

(b) an orbiter-penetrator mission costing $\$ 400-600 \mathrm{M}$. The specifics of the orbiter portion will depend on whether or not a NASA, ESA, or joint Mars orbiter flies first;

(c) an advanced surface mission (e.g., a lander/rover) costing above $\$ 600 \mathrm{M}$.

4. For consistency of planning, all costs should be taken to include spacecraft and instruments without tracking and launch costs. Roughly equal contributions are expected ultimately from each side. Balance of funding may be achieved by allocation of the responsibility for launch and tracking.

1. Determine the global elemental and mineralogical character of the surface.

2. Determine with high precision the global gravitational field and topography of the planet.

3. Establish the nature of the intrinsic and induced magnetic fields.

4. Determine the time and space distribution, abundance, sources, and sinks of volatile materials and dust over a seasonal cycle.

5. Establish the chemistry, energetics, and dynamics of the lower atmosphere.

6. Establish the structure, chemistry, energetics, and dynamics of the upper atmosphere and improve our knowledge about its isotopic composition.

7. Establish the structure, chemistry, energetics, and dynamics of the ionosphere. 


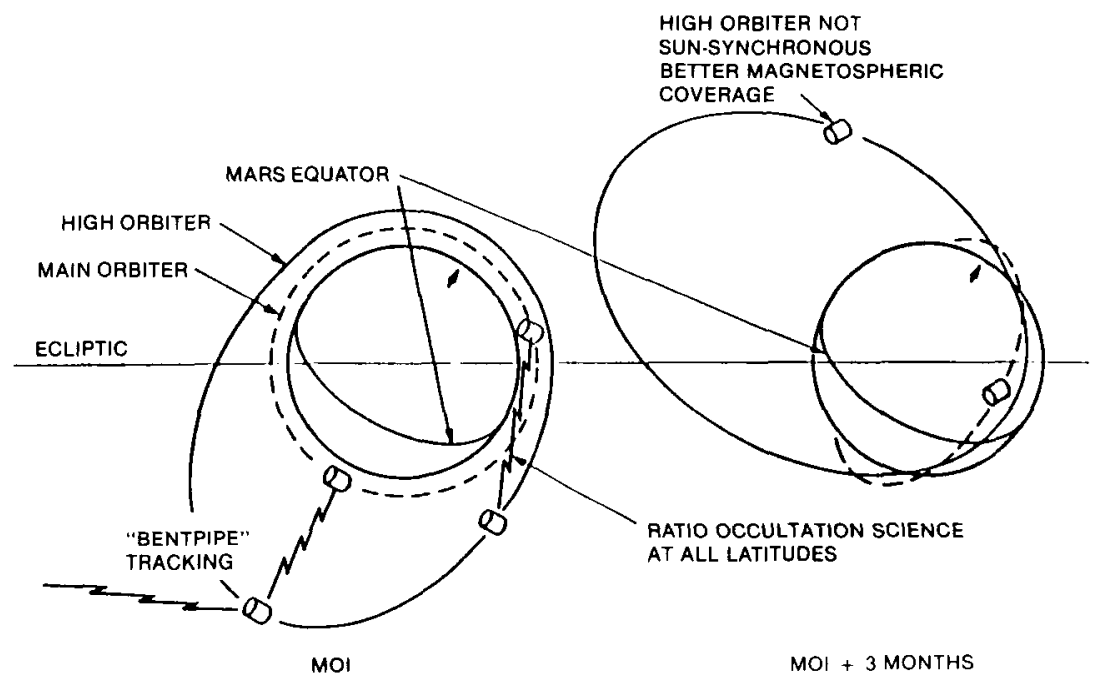

FIGURE A.1 Dual spacecraft Mars Orbiter geometry.

8. Determine the nature of the solar wind interaction with Mars and its environment.

These scientific objectives could be pursued by a variety of dedicated orbiting spacecraft. A circular, low-altitude orbit would be strongly preferred for those instruments that address objectives such as 1,4 , and 5 , whereas a spacecraft with a highly eccentric orbit is required for measurements dealing with objectives 3,6 , 7 , and 8. The science objectives of (2) can be pursued in either configuration (although the higher harmonics of the gravity field measurements are best measured from an orbit with low periapsis). There is, thus, strong scientific justification for both types of missions for the near-future exploration of Mars.

However, there are numerous and significant advantages of designing a mission consisting of two such orbiters operating simultaneously. Such a combined mission would result in scientific advances that would greatly exceed those which could be obtained by two successful but independent missions.

One of the major advantages of a dual orbiter mission is the capability of performing simultaneous in situ and remote observations of different regions of Mars and its environment. A prime example of this is tied to the objective of establishing whether 
Mars has an intrinsic magnetic field or not. The magnetic field, which is to be encountered even at low orbital altitudes, is anticipated to consist partially if not fully of induced fields. In order to be able to quantify the ionospheric and solar wind induced components of this field, one needs to make simultaneous measurements of the magnetic field and various plasma parameters at widely separated regions (e.g., outside the bow shock). Similar arguments hold for many of the high-priority atmospheric and ionospheric observations.

A dual orbiter mission would also dramatically improve observations of surface composition and dust distribution and transport, as well as $\mathrm{H}_{2} \mathrm{O}$ and $\mathrm{CO}_{2}$ variations over short and long periods, because of the temporal nature of the Mars environment. A global assessment of surface geology and composition over a 2-year period, for example, is closely coupled to the diurnal, seasonal, and sporadic variations of atmospheric phenomena (clouds, frost, airborne dust, and particle transport). The dual orbiter mission allows one of the spacecraft to map with high spatial resolution at close range, while the other provides a continuous synoptic view of the dynamics of the whole planet. The global compositional information is obtained from low-altitude measurements by the accumulation of high spatial resolution data over a period of time.

Two further examples of significant scientific improvements through a dual orbiter mission can be found in the radio science investigations. These improvements can be realized by providing a dual frequency link between the two spacecraft, which allows radio occultation measurements to be carried out over a much wider range of latitudes and local times than would be possible with just a single orbiter. Furthermore, the inclusion of such a dual frequency link provides the opportunity of using the "bentpipe" tracking technique, which leads to a significant improvement in the determination of the gravitational field at high latitudes.

It is anticipated that certain instruments would have to be carried on both orbiters if they are flown separately. This potential redundancy can be eliminated in a dual orbiter mission, leading to a more versatile and/or sophisticated payload complement for the same cost and weight commitment. 
Instrumentation

The required instrumentation for both spacecraft is based on existing technology, and some of the instruments are similar to those that have been flown on previous missions. The model payloads (Table A.3) differ somewhat from those of MGCO and Kepler in order to eliminate overlap (and, hence, increase the resources available for additional investigations) and to implement those experiments that require two spacecraft.

\section{Mars Surface Network}

Mission Description

This mission would consist of a single large orbiter, equipped with a probe carrier containing approximately twelve penetrators. The spacecraft would be placed in near-polar orbit around Mars, from where the penetrators would be released one at a time to land on selected targets on the surface. Each penetrator consists of a cylindrical body approximately $10 \mathrm{~cm}$ in diameter and 140 $\mathrm{cm}$ long (Figure A.2). On landing, the forebody enters several meters into the surface, and the afterbody remains on the surface (Figure A.3). The orbiter collects data from the penetrators after deployment and relays it back to Earth; it also carries a limited scientific payload in support of the surface network.

Several options exist within this basic mission design. The orbiter and probe carrier could be launched separately; the latter does not necessarily have to go into Mars orbit, but the range of targets for the penetrators and the accuracy of placement are then reduced. A case can be made for the use of nonpenetrating hard landers in place of penetrators, with a different emphasis on the scientific goals addressed. Finally, the penetrators themselves can be more or less sophisticated; the major trade-off is whether or not to use radioactive thermoelectric generator (RTG) power supplies in order to obtain lifetimes in excess of a Martian year. Battery-powered penetrators have lifetimes measured in weeks and were not recommended by the TPSG because the meteorology and seismometric objectives of the network, in particular, cannot be properly addressed in such a short time. A large quantity of literature exists on penetrator missions to Mars as a result of previous NASA and U.S. industry studies; these are available for reference, and the details will not be repeated here. 


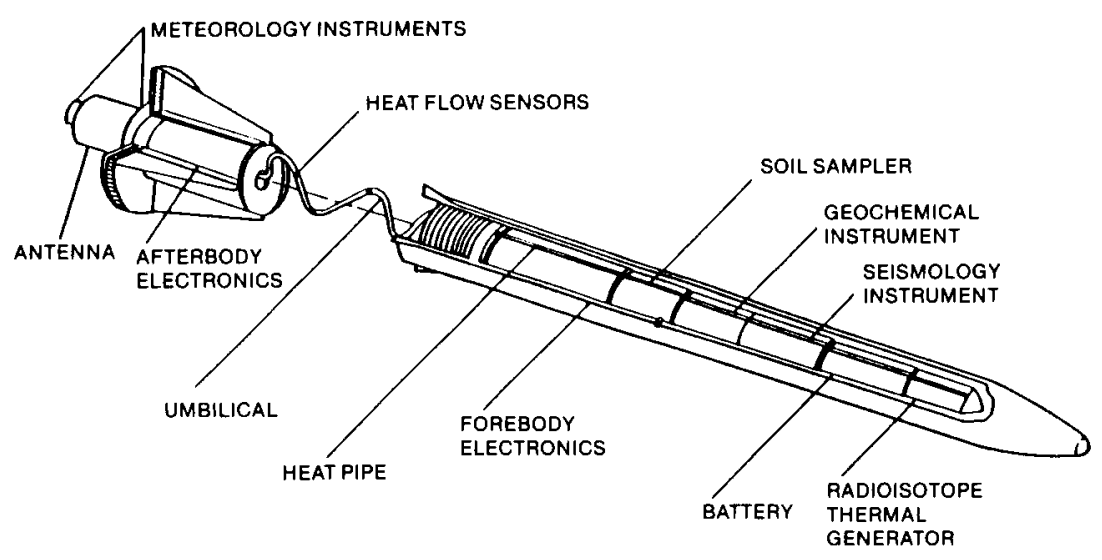

AFTERBODY HAS BEEN SEPARATED IN ABOVE VIEW FOR CLARITY

FIGURE A.2 Mars penetrator.

\section{Expected State of Knowledge of Mars After MDO or MGCO}

Although the Viking landers yielded valuable inorganic chemical data, this information comprised a partial elemental analysis at two surface sites and did not include direct mineralogical or phase compositional data. The Mars dual orbiter (MDO) mission would provide partial chemical and phase analyses on a global scale. Gravitational field data would be obtained, but no definitive seismic data relating to interior or crystal structure, or setting definite limits on the level of internal activity, are or will be available without another mission to land on the surface. Similarly, the dual orbiter mission will map the planetary magnetic field, but many questions about local variations will require surfacedeployed magnetometers.

Landed meteorology is so far limited to two sites, far fewer than the 10 or 12 considered the minimum for planetary-scale coverage, and Viking did not include atmospheric water measurements at the surface. These are required, in addition to global water measurements by remote sensing, to study the exchange of water between the atmosphere and the soil, and again a number of widely dispersed stations are required.

Both Mariner 9 and Viking provided valuable imaging and remote sensing data that would be massively extended by the dual orbiter. Following the latter, a start could be made on developing 
TABLE A.3a Core Instruments for Circular Orbiter

\begin{tabular}{|c|c|c|}
\hline Instrument & Expected Results & $\begin{array}{l}\text { Relevant } \\
\text { Science } \\
\text { Objective }\end{array}$ \\
\hline Gamma-ray spectrometer & $\begin{array}{l}\text { Surface elemental composition } \\
\text { ( } \mathrm{Si}, \mathrm{Al}, \mathrm{Mg}, \mathrm{Fe}, \mathrm{Ti}, \mathrm{Ca}, \mathrm{Na}, \mathrm{Ce}, \\
\mathrm{Th}, \mathrm{K}, \text { etc.). } \\
\text { Distribution of subsurface water, } \\
\text { thickness of } \mathrm{CO}_{2} / \mathrm{H}_{2} \mathrm{O} \text { ice caps and } \\
\text { composition of polar laminae. }\end{array}$ & 1 \\
\hline Mapping spectrometer & $\begin{array}{l}\text { Surface mineral composition-- } \\
\text { feldspar, pyroxene, olivines. } \\
\text { Measure frost distribution, } \\
\text { condensates, and bound water } \\
\text { in surface clays. }\end{array}$ & 1 \\
\hline $\begin{array}{l}\text { Pressure modulated } \\
\text { IR radiometer }\end{array}$ & $\begin{array}{l}\text { Vertical distribution of temperature } \\
\text { field, water vapor, dust content, } \\
\text { and properties and distribution of } \\
\text { condensates, clouds, and haze. }\end{array}$ & $\mathbf{4}, 5$ \\
\hline $\begin{array}{l}\text { Spectroscopic } \\
\text { imaging camera }\end{array}$ & Global high-resolution topography. & 2 \\
\hline $\begin{array}{l}\text { UV/visible spectrom- } \\
\text { eter/interferometer }\end{array}$ & $\begin{array}{l}\text { Abundance and velocity of neutral } \\
\text { species in the lower and upper } \\
\text { atmosphere. } \\
\text { Spatial and temporal variations of } \\
\text { dust content of the lower atmosphere. }\end{array}$ & $4,5,6$ \\
\hline $\begin{array}{l}\text { Radar altimeter/ } \\
\text { sounder }\end{array}$ & $\begin{array}{l}\text { Global figure and surface topography. } \\
\text { Polar cap stratigraphy; water in } \\
\text { regolith. }\end{array}$ & $1,2,4$ \\
\hline $\begin{array}{l}\text { Dual frequency, dual } \\
\text { spacecraft Doppler } \\
\text { instrumentation }\end{array}$ & $\begin{array}{l}\text { Global gravity field and its } \\
\text { anomalies. } \\
\text { Profiles of atmospheric and } \\
\text { ionospheric refractivity. }\end{array}$ & $2,3,7$ \\
\hline Magnetometer & Same as an eccentric orbiter. & $3,6,7$ \\
\hline
\end{tabular}


TABLE A.3b Core Instrumenta for Eccentric Orbiter

Relevant

Science

Instrument

Expected Results

Objective

Magnetometer

Neutral mass spectrometer

Ion mass spectrometer

Electron Langmuir probe

Retarding potential

analyzer

Plasma particle analyzer

UV/visible spectrometer/ interferometer

Three-channel imaging camera

Dual frequency, dual spacecraft Doppler instrumentation
Strength and orientation of intrinsic

$3,6,7$ and induced magnetic field. Spatial and temporal variations of induced magnetic fields.

Upper atmosphere density, composition (chemical and isotopic), temperature, and eddy coefficient.

Ion density and composition (chemical and isotopic).

7,8

Electron density and temperature.

7,8

Ion density, temperature, and velocity.

7,8

Three-dimensional velocity distribution

7,8 of electrons and ions.

Same as circular orbiter.

$4,5,6$

Global and regional images.

$1,4,5$

Monitoring of variations in surface

and atmospheric phenomena.

Same as circular orbiter.

$2,5,7$

and testing a Mars global atmospheric circulation model. Surface network measurements would then be sought to refine this. A significant advance on the characterization of the wide variety of geological sites observed from orbit (ancient cratered terrain, volcanic and sedimentary terrains, annual and residual polar caps, 


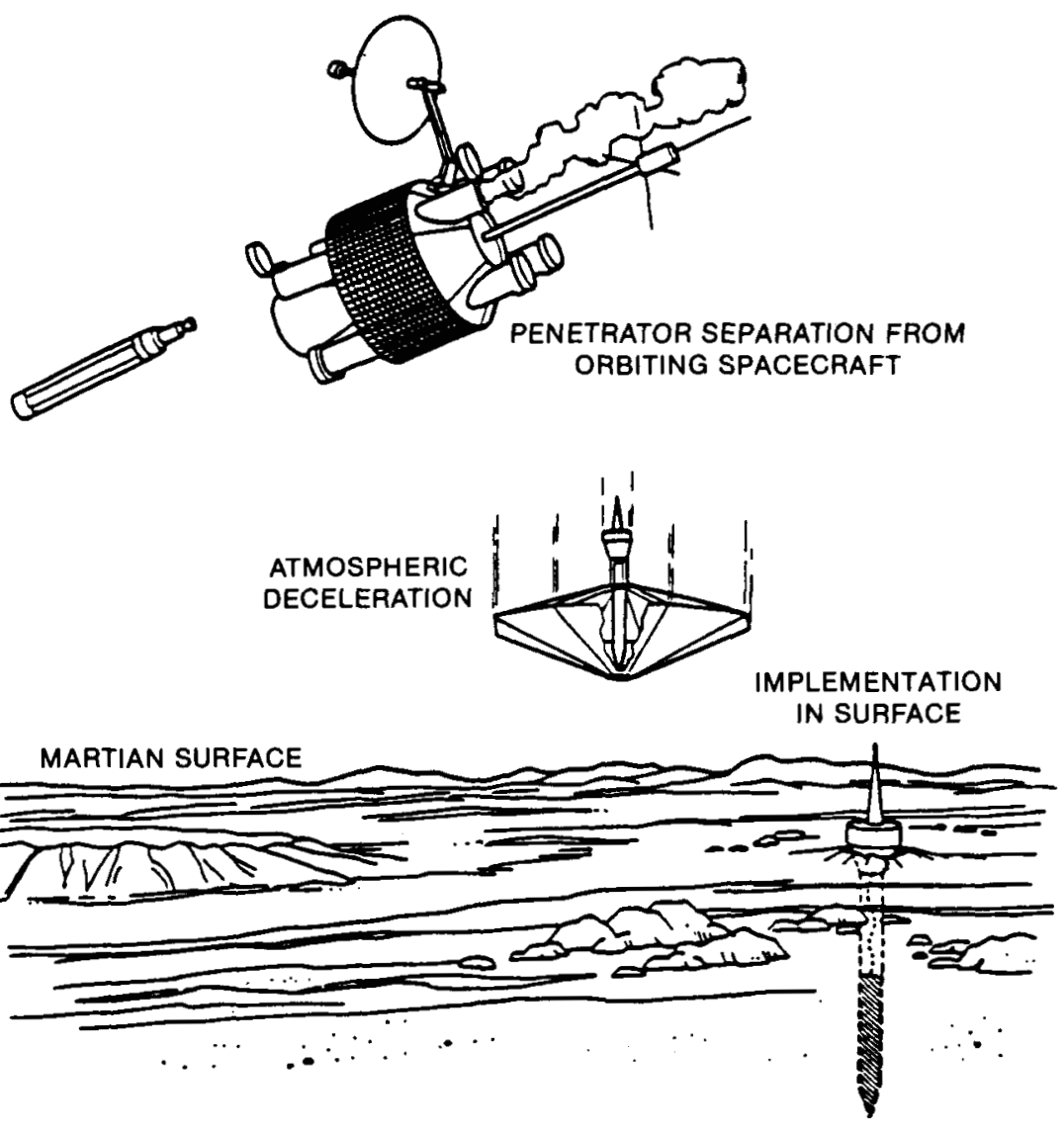

FIGURE A.3 Penetrator deployment on Mars.

fluid-formed channels, giant canyons, etc.) requires surface morphology and strength and stratigraphic measurements, again at various sites.

\section{Scientific Objectives}

Global Distribution of Igneous and Sedimentary Rock It is important to obtain as complete a chemical (elemental) analysis as possible not only on regionally defined (i.e., from orbit) terrain, but also on a variety of particular samples whose geological siting and textural characteristics are well documented. This analysis is critical for understanding the source and geological history of 
the surface material and for understanding the chemistry and processes in the source regions of igneous rocks. Igneous rock elemental analysis also allows the composition of derived sedimentary rocks to be interpreted in terms of weathering and transport processes. Previous landed analyses have not included the light elements $(z<11)$ and, therefore, make the interpretation of regolith processes involving interactions with and storage of volatiles difficult. Orbital gamma-ray measurements may relieve the problem on a global scale for $\mathrm{H}, \mathrm{C}$, and S. However, this must be related to geological setting, texture, and (especially) phase analysis. The latter is vital in that it allows full interpretation of the elemental analyses in terms of the origin and history of the sample. The same elemental assemblage can result in totally different mineral and phase assemblages, depending on origin. For instance, weathered or metamorphased rocks often cannot be distinguished on chemistry alone from their igneous or sedimentary source rocks.

Distribution of Subsurface Volatiles The abundance and distribution of regolith volatiles are critical because of their thermally stimulated mobility and potentially profound effects on Martian climate (through their influence on atmospheric pressure, etc.). The questions of the amount and availability of water as permafrost and the composition and mass of the permanent polar cap are specific important examples. Again, samples at a wide variety of sites, latitudes, and depths are required.

Interior and Crustal Structure A seismological study would yield information on three aspects of Mars: (1) level of seismic activity, (2) radial structure in local regions, and (3) detection of any seismic activity which would provide a first-order constraint on the internal dynamics.

Since it is evident that there are several tectonic provinces on Mars (e.g., Tharsis Bulge, north-south hemispheric boundary), no one site is sufficient to characterize the global seismic activity. Assuming Mars is seismically active, the variability of this activity, globally and regionally, provides strong constraints on the tectonic and internal dynamics. If the seismic activity is sufficiently great, then it is possible, using a minimum of four stations, globally distributed, to determine the internal constitution, including radial density distribution, size and physical state of the core, and possibly mantle phase transitions. With strategic placing of three 
seismographs, it is also possible to determine local crustal structure (e.g., the Tharsis Bulge region). Other information on local crustal structure will result from an analysis of the deceleration profiles of the penetrators themselves. Stratification of the surface, in particular, will be characterized by this technique.

Magnetic Properties Advances over orbital magnetometer studies are possible by simultaneous magnetic field measurements at ground stations and in orbit. It is only in this way that a clear separation of intrinsic field from time-variable field components can be made. The intrinsic field thereby determined provides constraints on the low-order harmonics of the global field and possibly some information on crustal remanent magnetization. Measurement of the field induced in the Martian interior by the time-variable external field components provides information on the internal electrical conductivity structure and, potentially, the deep thermal structure of the planetary mantle.

Meteorology and General Circulation The primary meteorological parameters to be measured are pressure, wind velocity, temperature, and water vapor partial pressure $\left(\mathrm{P}_{\mathrm{H}_{2} \mathrm{O}}\right)$. Measurement of the first three parameters at a large $(>10)$ number of sites over a Martian year provides crucial information for development of diagnostic global circulation models, particularly when supporting vertical temperature and humidity profile data are obtained by remote sensing from the orbiter. The $\mathrm{P}_{\mathrm{H}_{2} \mathrm{O}}$ measurements provide information on the regional and temporal behavior of water at the atmosphere-surface interface. Synoptic observations will yield diurnal and seasonal variability of $\mathrm{P}_{\mathrm{H}_{2} \mathrm{O}}$.

Heat Flow in the Crust Determination of the global heat flow would provide constraints on the radionuclide content and thermal structure and history of the interior. Regional variability of heat flow provides information on crustal thickness and tectonic regime. Since heat flow measurements require near-surface conductivity determination, an additional result is a partial characterization of the near-surface thermal transport properties.

Surface Imaging Imaging at each station is needed to document the local geological environment in which the sampling is taking place. Imaging information accumulated from all the stations would characterize the region and temporal variabilities of local geological environments. This would include detection of local 
condensation (e.g., $\mathrm{H}_{2} \mathrm{O}$ frost), which could be correlated with the $\mathrm{P}_{\mathrm{H}_{3} \mathrm{O}}$ measurements and dust opacity and movement monitoring, for example. For the study of transient phenomena, especially dust storms, synoptic support imaging from the orbiter is desirable.

Aeronomy In the event that MGCO is not accompanied by an aeronomy orbiter, this science could be accommodated on the orbiter supporting the surface network. If the full dual orbiter mission is flown as recommended, it still would be valuable to make new aeronomy measurements at a different point in the solar cycle and at the same time as surface magnetic field measurements. In this context, aeronomy includes compositional studies of the atmosphere using mass spectrometer and UV and IR spectroscopy to monitor photochemical behavior.

Instrumentation

Table A.4 lists model payload instruments for the network mission. The accommodation inside a penetrator is rather limited, and some careful trade-offs would be necessary if all of the experiments listed were to be included. It is unlikely that all of the experiments would be included in all of the penetrators, for instance. In one important case-the heat flow experiment-the study group was of the opinion that a proven technique did not exist at present that could perform these important measurements. In all other cases, feasibility is reasonably well established, including the ability to withstand the $20,000-\mathrm{g}$ shock of landing. Heat flow measurements are important, and a strong case exists for developing a working technique.

Several compact, simple, shock-resistant chemical analysis devices have been shown to be compatible with utilization in penetrator forebodies. These include alpha-backscatter and x-ray fluorescence, which require primitive sample acquisition or exposure of the sample to the source of the instrument. Other instrumentation, such as neutron/alpha-ray spectrometry, can be employed within the penetrator body without sample acquisition. Although each has its own analytical strengths and weaknesses, they generally provide analysis extending to minor/trace elements, and abundances of regolith volatiles such as $\mathrm{H}$ can be determined. In the case of the penetrators, analysis at depth (>1 meter) is possible. The sites of water in condensates and various mineral lattices can be assayed using slow heating and a fused water analysis. 
TABLE A.4 Mars Network Mission: Model Payload

\section{On Penetrators}

1. Pulsed neutron source/gamma-ray spectrometer.

2. alpha-backscatter/x-ray fluorescence (backup for (1), needs lateral sampling)

3. Seismometer (crustal structure, crustal stresses, internal structure, size of core)

4. Magnetometer (intrinsic field, electromagnetic induction, internal conductivity)

5. Hygrometer (subsurface $\mathrm{H}_{2} \mathrm{O}$. Requires lateral sampling)

6. Accelerometer (subsurface strength and stratigraphy, depth of penetration)

7. Meteorology package (wind speed and direction, temperature, pressure, humidity)

8. Imaging (surface morphology, meteorology)

9. Heat flow (thermal properties of crust, magnitude of radioactive heat source)

\section{On Orbiter (supporting measurements for network)}

1. IR radiometer/spectrometer (atmospheric thermal structure, composition)

2. Magnetometer (external field)

3. Aeronomy package

\section{Other Orbiter Science}

1. Electromagnetic sounder

2. Radar

\footnotetext{
${ }^{a}$ Development essential to establish feasibility.
}

Hard landers may provide opportunities for more sophisticated phase analysis instrumentation, although not at such depth as is possible with penetrators.

Sufficiently compact, lightweight, and rugged seismometers exist and could be deployed by a hard lander or penetrator. An example of such an instrument is the combined bubble tiltmeter and force balance accelerometer currently being developed at Caltech. Penetrator deployment is likely to provide better coupling of the seismometer, although adequate coupling may be achievable by deployment from a hard lander. The likely data rate for a seismically active region can be accommodated. 
A suitable magnetometer instrument would consist of three orthogonal fluxgate magnetometers located either at the surface or subsurface. Care must be taken to ensure separation of planetary fields from fields provided by the electronics or permanently magnetized part of the landed spacecraft. A similar instrument would be installed on the orbiter.

Two measurements are required to determine heat flow: the thermal conductivity and the temperature gradient. The former can be determined using the transfer of heat away from a known heat source (the RTG, for example) in the forebody of the penetrator. This experiment may not be feasible with a hard lander. The regional temperature gradient is difficult to determine because of contamination problems from other heat sources but might be determined from temperature sensors distributed along the penetrator forebody and umbilicus.

Small rugged instruments exist that are compatible with both penetrators and hard landers and are capable of measuring the key meteorological parameters, i.e., pressure, temperature, humidity, and wind velocity. Temperature can be measured with a thermocouple, wind velocity using an ion-flow technique, and pressure by diaphram deformant using a strain gauge bridge. Humidity can be determined using a hygroscopic detector or the sensitivity of the vibrational frequency of a quartz crystal to moisture.

Imaging may be achieved by a small charge couple device (CCD). Such arrays have been tested at high acceleration loads.

\section{Mars Surface Rover}

Mission Description

The rover mission consists of two and possibly three independent vehicles and one relay telecommunication satellite. For a mission in the cost envisaged here (1000M) the rovers would not be intelligent robots but would be controlled from Earth using television for guidance. A moderate level of autonomy is not ruled out, however, and there is scope for considerable ingenuity in designing a low-cost rover that can cover the maximum distance per day, avoiding or scaling obstacles.

All vehicles including the communications satellite are sent to Mars by a single shuttle launch, with a Centaur stage. The communications satellite carries minimal science. It can be placed either 
on an equatorial geosynchronous orbit or on a 12-hour eccentric orbit optimized for maximum visibility of the rovers. Telemetry data from the rovers and ground commands from Earth transit by the satellite. The rovers are placed in individual aeroshells and are independently delivered from Martian orbit on distant preselected locations on the planet.

A major uncertainty on the cost is introduced today by the difficult choice of a landing system. Viking-type soft landing systems are well understood, but their price seems to be exceedingly high (500M). Semihard landing systems can be imagined for this mission (Figure A.4) with satisfactory specifications and would be much cheaper, but no study or costing has been performed on these systems. The rovers can be of different types, varying from the JPL Mars 84 rover (Figure A.5) to the Centre National d'Etudes Spatiales (CNES)-ESA Mars Ball (Figure A.6). As of today, a two wheeled vehicle (like the Ball) seems the most likely solution. All types would have a mass around 350 kilograms and would carry 100 kilograms of scientific instrumentation. They would be powered by two 50-watt RTG's and would have a communication capacity of 109 bits per day. The rovers would be semi-autonomous: the sequence of their motion would start by pictures being sent to Earth defining the nature of the terrain and the landscape. Then a strategy of displacement over the known terrain would be elaborated at the control center and the rover ordered to move all the way to the horizon, where it would stop and the sequence would be repeated.

Two modes of utilization would be used:

1. Each rover carries a series of attached instruments (see Table A.5) that are operated when the rover stops on orders sent from Earth. The rover can remain at rest for any chosen duration (hours to weeks).

2. Each rover carries a series of independent stations, which are detached and left behind in order to create a network (meteorological and seismic).

The possible range of each rover is over 1,000 kilometers in one year.

\section{Scientific Objectives}

A rover mission is a mobile laboratory and therefore has the capacity of visiting various terrains; its main scientific objective 


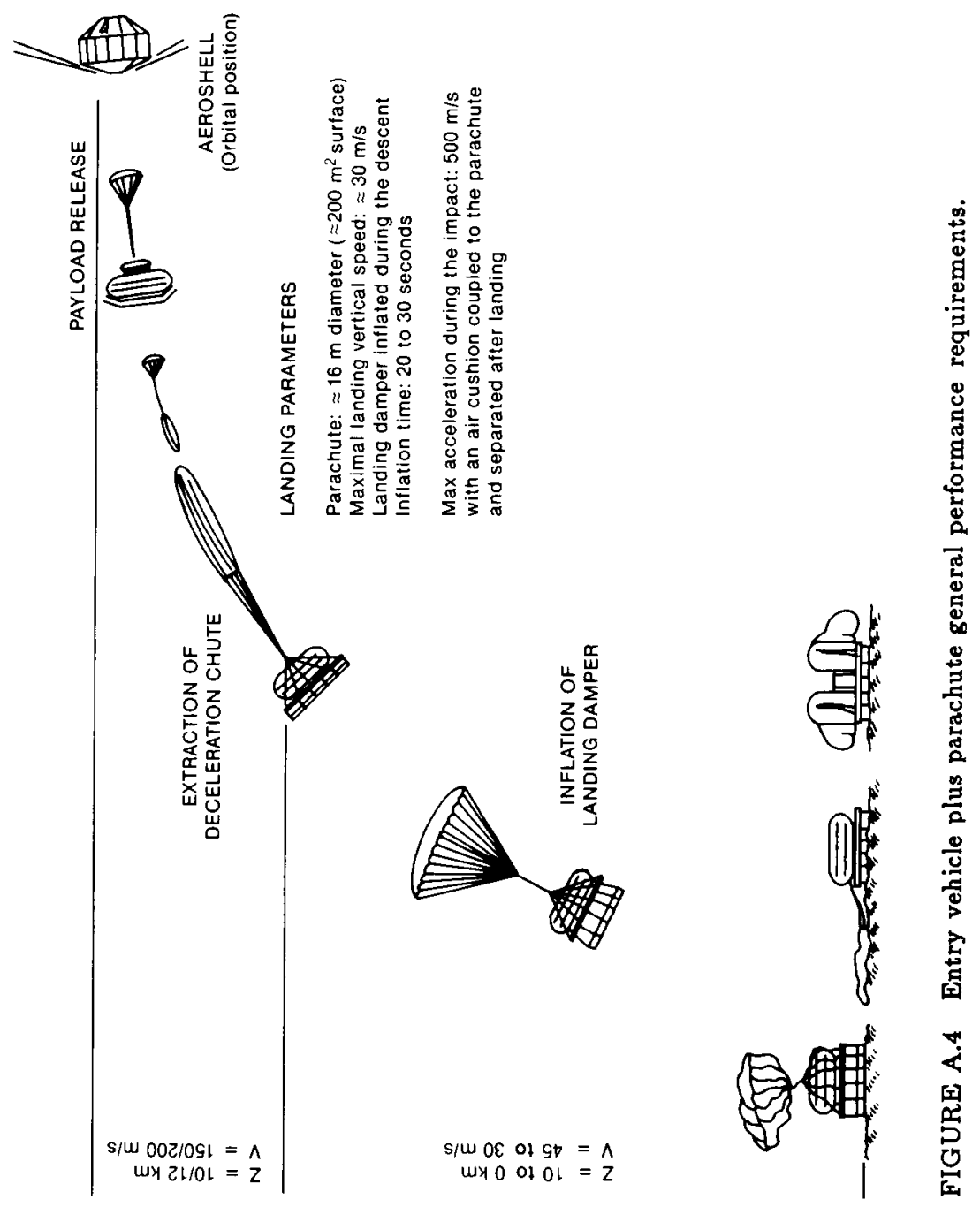




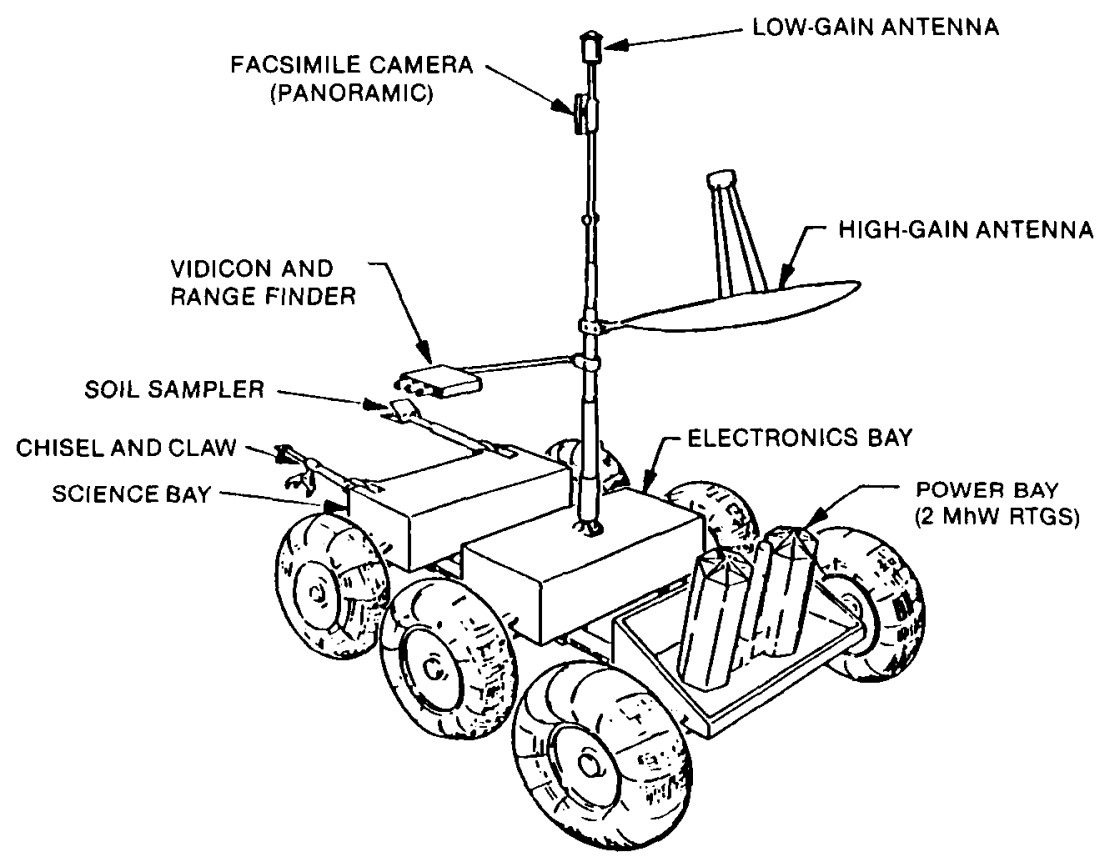

FIGURE A.5 Jet Propulsion Laboratory Mars 84 rover.

is to study the geology of Mars in all its aspects: history from primordial events to the situation of today, tectonics including volcanism, petrology, stratigraphy, climatology past and present, and weathering of the surface.

Of essential importance is the characterization of water: since it is thought that the oceans of Mars lie frozen below the surface, water will be searched for down to a depth of 1 kilometer.

Many Mars regions have been identified where geologic units of several types occur in limited areas. The Mars Geoscience Climatology Orbiter will verify these recurrences and identify other areas where there are multiple targets in small areas. The rover is the device used to investigate and analyze rocks, soils, and frozen volatiles in such areas. For example, layered rocks in the canyons offer attractive targets that can be identified and characterized in short distances. Alternatively, a landing in the polar area would allow analyses to be carried out of the Northern Plains rocks, the polar layered deposits, the permanent and seasonal ice structures, and earlier accumulations in a limited area. 


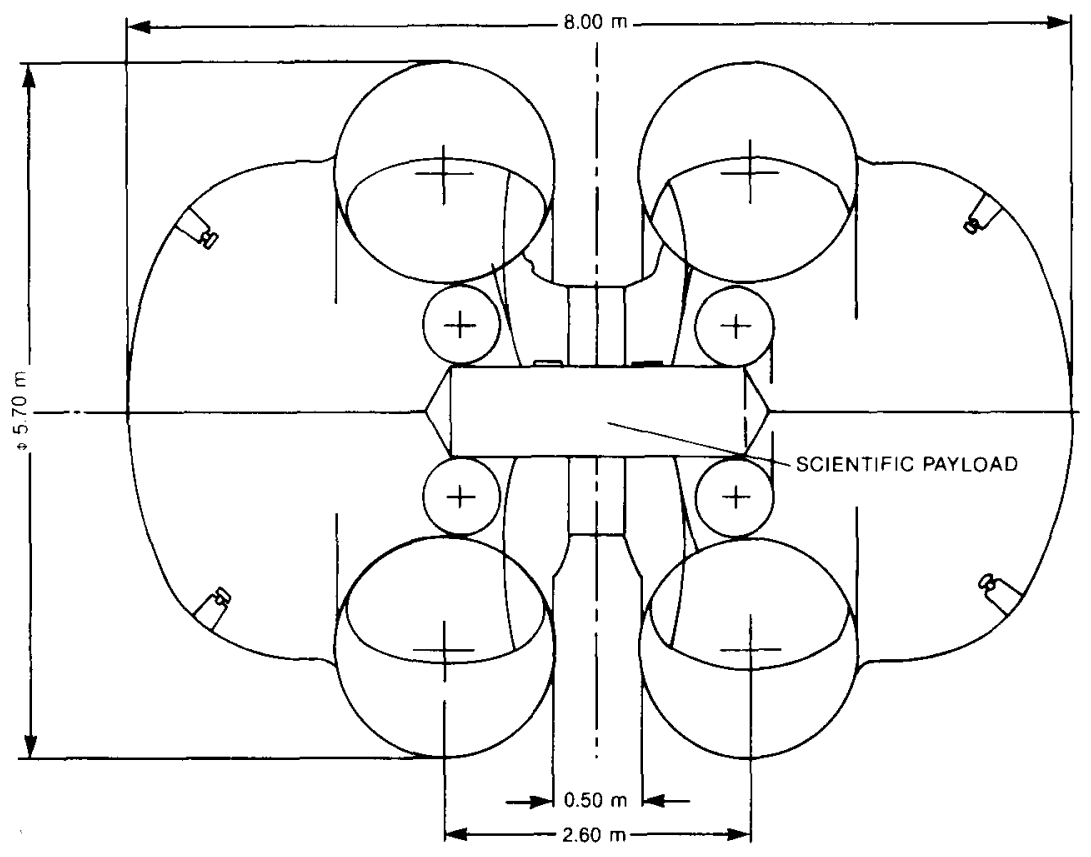

FIGURE A.6 Centre National d'Etudes Spatials-ESA Mars Ball.

Isotopic Analysis Determination of isotopic ratios that will be performed by neutron activation analysis is a strong clue to chemical evolutionary history. It may also be possible to deduce isotopic data from rocks formed at different times in history from argon isotope ratios. This will establish the impact flux, then a basis for the discussion of the thermal history and of the significance of possible atmospheric changes. Even crude observations will provide the scale forthe stratigraphy of observed terraces.

Chemical Analysis Chemical analysis of samples will provide the basis for understanding the geological and geochemical processes. The major element composition is related to petrology, and trace elements to rock genesis.

In combination with age determination, the compositional analysis of the lava will provide information on the degree of differentiation of the planet, the composition of the source material at depth, and the thermal evolution. Abundance of elements like $\mathrm{Ni}$ and Co will give information on the composition of the mantle to be compared with the Earth's. 
TABLE A.5 A Mars Rover Model Payload

\begin{tabular}{|c|c|}
\hline Payload & Kil \\
\hline Argon dating and neutron activation analysis & 20 \\
\hline $\begin{array}{l}\text { Chemistry and mineralogy of surface samples } \\
\text { (integrated tracking microscopy, energy } \\
\text { dispersive } x \text {-ray analysis, Auger electron } \\
\text { spectroscopy, } x \text {-ray photoelectric spectroscopy) }\end{array}$ & 17 \\
\hline Rock drill & 8 \\
\hline Imagery & 5 \\
\hline Electromagnetic sounder & 7. \\
\hline Magnetometer & 2. \\
\hline Meteorology package & 5 \\
\hline Seismometry (active and passive) & 30 \\
\hline TOTAL & 95 \\
\hline
\end{tabular}

A large number of samples will be analyzed with great accuracy, originating either from the surface or from a depth of 1 to 2 meters.

Identification of Mineral Phase Interpretation of the chemical analysis of rocks is not possible without the knowledge of mineral phases.

With igneous rocks, this knowledge is needed for determining the conditions under which the rock crystallized. Composition of the crystal phase when this phase is identified places constraints on the style of differentiation, which in turn informs us of planetary internal processes.

With weathered rocks, study of clay minerals and other alteration products is necessary to understand the weathering processes.

Mineral phases will also be identified through analysis of a large number of samples by various crystallographic techniques.

Stratigraphic Relations and Ages Time of emplacement, thickness, and texture of layers in combination with the composition 
contain information about periods of volcanic activity, its regional dependency and duration. The nature, duration, and area of occurrence of erosional and depositional cycles are clues to exogenic processes.

Surface terrains on Mars represent the last layer of a series of geological units. Regolith, lava flows, weathered areas, and fine-grained sediments are piled up on each other, and this stratified structure can be quantified by observing vertical profiles even from the outside. Processes such as erosion, volcanic eruptions, products and deposits are documented in a variety of morphological landforms. A moving vehicle would investigate, for varying viewing angles, the location and nature of the contact of all morphological units and allow identification of individual layers in walls as well as grain distributions.

Textural Studies Textural studies provide the basic data for determining the origin (fluvial, eolian) and source of the sediments. Grain size distribution and grain shape analysis are diagnostic of various erosional and transportational processes. A knowledge of the way mineral grains are weathered, mechanically broken, and transported is needed for the interpretation of geochemical surveying from orbit. It may be possible to determine times of fluvial episodes by dating rocks above and below boundaries that were developed during water-rich periods.

For igneous rocks, the form of the crystal together with bulk chemistry provide a clue for the interpretation of the cooling history of the rock.

Internal Structure and Tectonics Knowledge of the interior is essential to establish the thermal history and bulk composition of Mars. Whereas the improvement of our knowledge of the global isostatic response of the lithosphere to topographic loads, as coming from the growing field of topography determined by classical orbiter science, brings information only on the upper layer of the planet, a good model of the interior can only be provided (1) from the determination of the precession constant by monitoring the change of position in space of fixed points at the surface, which yield a value of the $\mathrm{C} / \mathrm{MR}^{2}$ parameter, a fundamental number for all differentiated models, or (2) from the recording of seismic events on a global basis, local measurements of active seismic events complementing the picture in giving finer details of the 
crust inhomogeneities. Such determination will enable scientists to address the following problems:

- origin of the center of mass-center of figure offset;

- departure from hydrostatic equilibrium (as confirmed by global gravity and topography determined from an orbiter) and permanent stresses exerted in the whole body;

- climatic changes on geological time scales due to resonant nutations, the amplitudes and periods of which depend on the precession constant;

- seasonal variations of the $\mathrm{CO}_{2}$ deposit on the polar caps as detected by changes in the sidereal rotation period of Mars directly mapped from the fixed points positioning in an absolute reference frame; and

- polar motion and forced nutations, which reflect the global elastic properties of the body and may reveal the existence of a liquid core.

Thermal, Magnetic, and Mechanical Studies Thermal, magnetic, and mechanical studies are the necessary complement to internal structure modeling. Physical quantities to be measured are as follows:

- heat flux (global and local), which brings constraints on (1) the thermal history (differentiation processes during the early history of Mars, rate and modalities of cooling, (2) the crust formation, (3) the surface modification processes, (4) the storage of degassed volatiles below the surface and the formation of permafrost;

- regolith conductivity and specific heat, coupled with the heat flow data, to yield understanding of regional geomorphology, meteorology, and soil-atmosphere interaction, such as the buffering role of the regolith in the seasonal and secular exchanges of volatiles between the polar caps and the atmosphere;

- magnetic characteristics of the soil samples (ferromagnetic, paramagnetic, and diamagnetic behavior) and residual magnetism, which will yield information on (1) paleomagnetism (past history of the planet) and (2) induced magnetism (related to the impacts that altered the properties of the target material); and

- response of Martian rocks to stresses, which will characterize the strength of the materials and therefore supplement the elastic lithosphere models. 
Permafrost Characterization The presence of a large reservoir of water stored in the form of permafrost down to a depth of hundreds of meters may explain many geomorphological structures such as the shape of the ejecta blankets, the structure of landslide debris, and the broad channels originating in collapsed terrains.

A key objective for the mission is to detect the presence of a subsurface permafrost, determine its thickness, and detect the location of the in-depth solid-liquid transition. This last aspect would also provide the near-surface thermal gradient. The sounding of the top kilometer region can best be accomplished by two complementary experiments: the sounding by electromagnetic waves and an active seismometry network. This combination can also yield the depth of the bedrock, the density of the regolith and the near-surface layering structure.

Meteorological Observations at the Surface Level A network of small meteorological stations will characterize the atmosphere at the surface, including pressure, temperature, wind speed and direction, soil moisture, atmospheric opacity at various wavelengths, and incoming and outgoing heat fluxes.

Comparison with Penetrator Network

The advantages of the rover mission are as follows:

- sophisticated instrumentation providing a complete characterization of each site,

- observation of the landscape,

- large number of documented adjacent sites, and

- choice of sites and samples.

Among experiments that can be carried by the rover and not by the penetrator network are the following:

- landscape description and geomorphology,

- examination of sequence of rocks providing good stratigraphy,

- study of permafrost,

- isotopic analysis providing crude age dating, and

- mineralogical and textural studies. 
Instrumentation

In general the accommodation for instruments on a rover is more generous in terms of mass and power than it is on a penetrator or hard lander, and the requirement to withstand the landing shock is far less severe. A model payload that would perform the investigations outlined in the section on scientific objectives above is given in Table A.5. Other exciting measurements could be made by the following instruments not included in the model payload: IR transmittance/reflectance spectrometer, calorimetric soil heating effused volatile analysis, retroreflector deployment for Very Long Baseline Interferometry (VLBI). 


\section{COST SUMMARY}

The costs of the missions in the prime set are summarized in Table A.6. The first total is the net cost of the mission computed according to the JWG guidelines, i.e., excluding launch and mission operations (tracking, etc.). The rover costs exceed the maximum guideline for an "expensive" mission (defined as 600 to $1,000 \mathrm{M}$ ); however, the study group considered that the rover represented the next logical step in a family of missions graduated by cost, and the least expensive, scientifically effective rover mission costs in the region of $1,200 \mathrm{M}$, if it has to be soft landed on the surface.

It appears likely that an inflatable rover such as the CNES Ball could be semihard landed, and this would be much cheaper. A very rough estimate of the cost for this has been made and is also shown in the table. 
TABLE A.6 Cost Summary for Recommended Missions

\begin{tabular}{|c|c|c|c|c|}
\hline & $\begin{array}{l}\text { Mars } \\
\text { Dual } \\
\text { Orbiter }\end{array}$ & $\begin{array}{l}\text { Mars } \\
\text { Surface } \\
\text { Network }\end{array}$ & $\begin{array}{l}\text { Mars Rov } \\
\text { Hard } \\
\text { Landing }\end{array}$ & 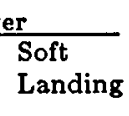 \\
\hline Orbiter 1 & 85 & 100 & 100 & 210 \\
\hline Orbiter 2 & 70 & - & - & - \\
\hline 12 Penetrators & - & 160 & - & - \\
\hline 2 Landers & - & - & 60 & 60 \\
\hline Landing system & - & - & 200 & 480 \\
\hline Integration & - & - & 50 & 70 \\
\hline Science & 65 & 75 & 140 & 140 \\
\hline Project management & 30 & 45 & 50 & 50 \\
\hline Subtotal & 250 & 355 & 600 & 1,010 \\
\hline Contingency $(20 \%)$ & 50 & 70 & 120 & 200 \\
\hline Total & 300 & 450 & 720 & 1,210 \\
\hline Launch & 115 & 150 & 50 & 150 \\
\hline Mission operations & 30 & 75 & 120 & 120 \\
\hline Subtotal & 145 & 225 & 270 & 270 \\
\hline Grand Total & 445 & 675 & 990 & 1,480 \\
\hline
\end{tabular}

NOTE: All numbers are millions of dollars. 


\section{OPTIONS FOR ESA-NASA DIVISION OF RESPONSIBILITIES}

The ESA-NASA division is clearest for the case of the dual orbiter mission, since a circular stabilized orbiter project (the MGCO) has been developed in the United States, while an eccentric "aeronomy" orbiter (Kepler) has recently completed a phase A study in Europe. At the time of writing, the most probable scenario for implementation of MDO is a NASA new start for MGCO in fiscal year 1984, leading to a 1990 launch, followed by a 1985 new start by ESA for Kepler, leading to a 1992 launch. Although not a truly joint mission, this would accomplish most of the scientific goals extolled above and would be steered by a joint scientific project group. More "jointness," plus cost savings, would result from a single launch of both orbiters. This approach is not compatible with the short-term plans of either agency at present, however, and should be contemplated only in the case that neither MGCO nor Kepler moves forward in its present form.

For the Surface Network mission, a wider range of options is possible, but it is clear that most of the experience with penetrators and hard landers, especially those using RTG power, resides in the United States. Hence, it seems logical, but not essential, to envisage a European orbiter transporting a payload of Americanbuilt penetrators with common science experiments. In costing, a single Shuttle-Centaur launch has been assumed, but a cleaner division of technical interfaces may be accomplished by launching the orbiter/relay and the penetrators separately.

The Rover mission is the most difficult to divide. Possibly two identical rovers could be built to a common design on opposite sides of the Atlantic, although obviously with a cost penalty. The most logical option might be ESA-built rovers and orbiter on a NASA-developed delivery system, since the latter is likely to require Viking heritage. 


\section{RECOMMENDATIONS AND TIMESCALES}

On a relatively short timescale, the best way to implement a joint mission of very high scientific priority is to transmute the existing NASA MGCO and ESA Kepler missions into the Mars Dual Orbiter project described above. In practice, this probably means securing a new start for Kepler as rapidly as possible in order to place it in a compatible time frame with MGCO, which seems to be on target for a 1990 launch. A launch of Kepler in 1990 now looks impossible, but a 1992 launch would still give valuable overlap, and a delay to MGCO, though deplorable, cannot be ruled out. A joint science steering group should be set up and coordinated payloads developed.

In addition to the above short-term initiative, a joint preproject study should be initiated on one or both of the two more expensive missions, with a view to starting phase $A$ and subsequent phases as soon as possible. Ideally, all three of the study group's recommended projects would be implemented on the timescale of Figure A.7, which is the shortest one thought to be practical. However, it should be noted that the network mission is not considered an essential precursor to the rover mission. If MGCO, but not Kepler, flies in the period around 1990, then the orbiter supporting the penetrators/hard landers or the rovers should be modified for aeronomy by having an eccentric orbit and a suitable scientific payload. 


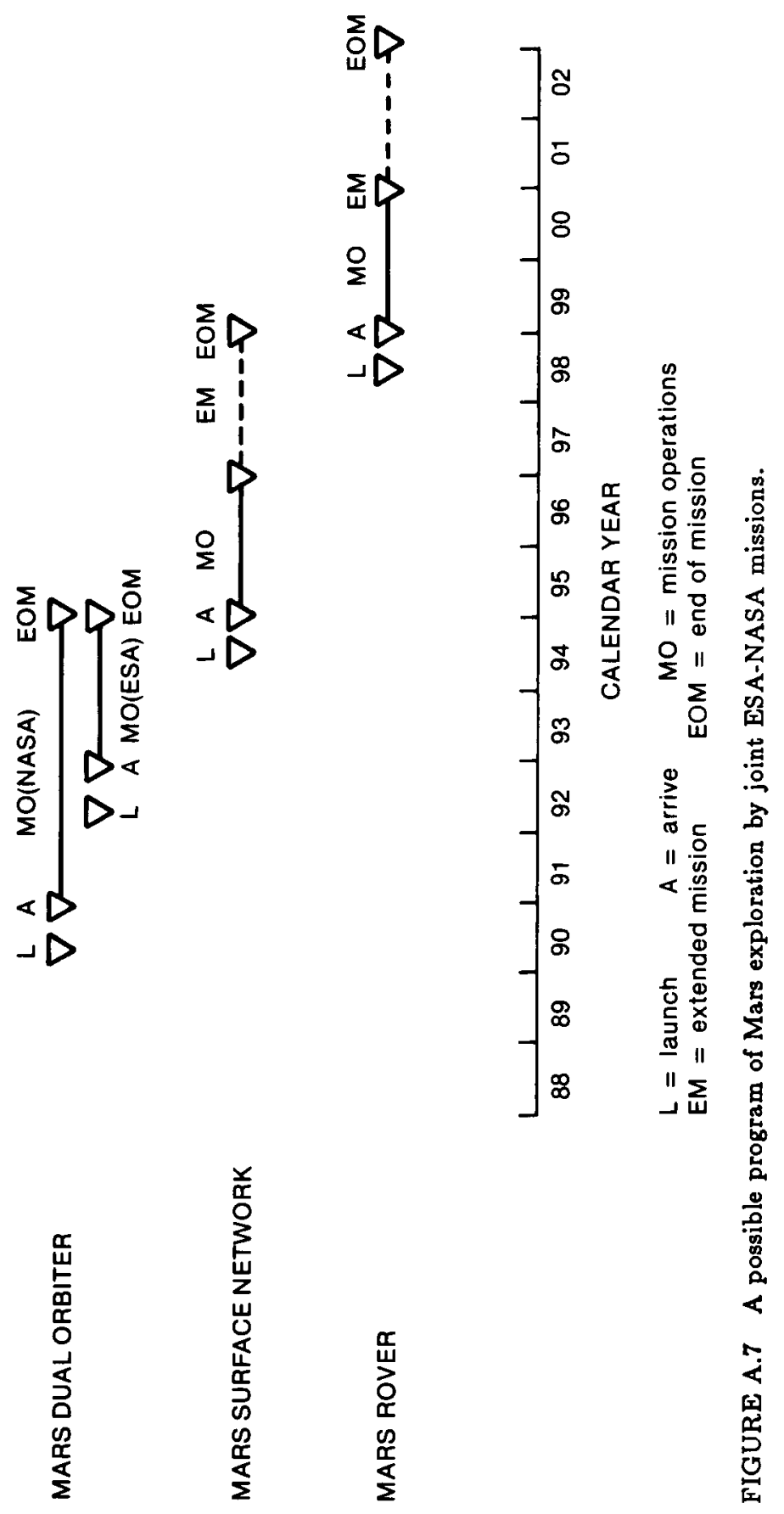




\section{APPENDIX B: OUTER PLANET MISSIONS}

\section{Outer Planets Study Team}

Frederick L. Scarf (Chair), TRW Space Systems Group Hans Balsiger, Physikalisches Institut der Universität Bern Angioletta Coradini, Istituto Astrofisica Spaziale Daniel Gautier, Observatoire de Paris

Eberhardt Grün, Max-Planck-Institut für Kernphysik Harold Masursky, U.S. Geological Survey

Tobias C. Owen, State University of New York at Stony Brook Alvin Seiff, NASA Ames Research Center

David J. Southwood, Imperial College of Science and Technology Darrell F. Strobel, Naval Research Laboratory

\section{Other Participants in Meetings}

Representatives of the JWG, Members of other study teams

J. Blamont, Paris
A. Brahic, Paris
H. Fechtig, Heidelberg
M. Fulchignoni, Rome
W. Ip, Lindau
E. Levy, Tucson
D. Morrison, Hawaii

Representatives of ESF-NAS/NRC

D. Kastel, NAS/NRC

K. Saul, ESF 
Representatives of NASA-ESA
A. Diaz, NASA
G. Haskell, ESA

Technical/scientific support
A. Amar, Centre National d'Etudes Spatiales
J. Beckman, Jet Propulsion Laboratory
J. Casani, Jet Propulsion Laboratory
R. Diehl, Jet Propulsion Laboratory
A. Frauenfelder, Science Applications, Inc.
T. Johnson, Jet Propulsion Laboratory
J. Lunine, California Institute of Technology
B. Mitchell, Jet Propulsion Laboratory
J. Neihoff, Science Applications, Inc.
K. Nock, Jet Propulsion Laboratory
A. Perret, Centre National d'Etudes Spatiales
A. Simon, Centre National d'Etudes Spatiales
B. Swenson, NASA-Ames Research Center 


\section{CONTENTS}

EXECUTIVE SUMMARY

INTRODUCTION

SCIENCE OBJECTIVES

MODEL PAYLOADS

STATE OF READINESS OF INSTRUMENTATION AND SUBSYSTEMS

SPECIAL CONSIDERATIONS/LAUNCH OPPORTUNITIES

COSTS AND SCHEDULE

BACK-UP MATERIAL FOR GALILEO COST ESTIMATE 127

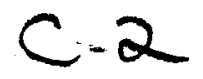




\section{EXECUTIVE SUMMARY}

The Outer Planets Study Team recommends a cooperative U.S.-European orbiter and probe mission to the Saturn system, using a Galileo-class spacecraft and a newly designed lightweight Titan probe.

The Saturn Orbiter and Titan Probe mission will provide important new information on the chemical composition of Titan's atmosphere and the nature of its surface, the atmosphere of Saturn, the structure and dynamics of the ring system, the characteristics of the icy satellites, and the behavior of the magnetosphere and the associated gas.

This combined orbiter and probe mission will fully address the eight fundamental science issues discussed in the Cassini proposal. These issues can be summarized in terms of the need to determine the following:

- the thermal structure and composition of the atmosphere of Saturn and their possible impact on theories of formation of the solar system, evolutionary histories of the planet, the rings, and the satellite system;

- atmospheric dynamics and the general circulation of a rapidly rotating planet, which obviously exhibits significant differences from Jupiter;

- dynamo theory and the generation of the axially symmetric magnetic field;

- the configuration and dynamical evolution of the ring system and its interrelation with the satellite system;

- the nature of the surface of Titan and its atmospheric composition leading to important constraints on theories of formation of the Saturn system;

- the detection of prebiotic molecules in Titan's atmosphere, and possibly the determination of physiochemical processes that lead to their formation;

- the formation, internal configuration, and surface processes of icy satellites as well as their comparative study; and 
- the configuration, composition, and dynamics of the magnetosphere of Saturn and its interactions with the solar wind, the satellites, and the rings.

As a Saturn orbiter, a Galileo-class spacecraft already has dual-spin design, so that particle investigators can obtain complete angular distributions, while imaging instruments and spectrometers can be precisely pointed. The Galileo imaging system has very high resolution, with image motion compensation and high data rate capability. There is a large-capacity tape recorder, a distributed data system, and a capability to carry the necessary science payload, including a Titan radar mapper. The spacecraft is designed to carry a probe, it has a probe relay antenna with pointing system and a relay link, and it also has a suitable orbit injection motor, plus an orbital maneuvering system with a large propellant capability.

The new lightweight Titan probe can provide continuous detailed measurements from above the haze layer to the surface over an extended period. The mission will yield information on the fundamental chemical composition of Titan's atmosphere, the nature of the clouds and haze, and the characteristics of the unseen surface.

The scientific advantages of a combined Saturn Orbiter and Titan Probe mission are great. Repeated close flybys of Titan can provide invaluable information of the Titan-plasma interaction and the Titan atmosphere and surface (from radio occultation and remote sensing). During each close pass of Titan, the large mass of this satellite permits a drastic modification to the Saturn orbit, leading to unparalleled opportunities to image Saturn and its ring system from high latitudes, to explore dynamical phenomena in Saturn's magnetosphere, and to investigate several of the small icy satellites.

The cost advantage associated with use of an existing spacecraft is also impressive; it has been estimated by the Jet Propulsion Laboratory (JPL) that it would cost approximately 3 to 3.5 times as much to develop a new orbiter with comparable capabilities. 


\section{INTRODUCTION}

At the second meeting of the Joint Working Group (JWG), October 1982, study teams concerned with missions to the terrestrial planets and primitive bodies were set up, but the Outer Planets Study Team was not formed until after the third JWG meeting (February 1983). This late decision to form a third study team was influenced by several events that occurred after October:

1. In November 1982 a group of 29 European scientists submitted a proposal to the European Space Agency (ESA) for a Saturn Orbiter and Titan Probe mission to be conducted jointly with the National Aeronautics and Space Administration (NASA). The coordinators of this proposal, referred to as Project Cassini, were D. Gautier and W.-H. Ip; the text contained a comprehensive summary of the significant scientific issues that were outstanding after the Pioneer 11 and Voyager 1 and 2 flybys of the SaturnTitan system. The Cassini proposal showed conclusively that a Saturn orbiter (with Titan radar mapper), together with a Titan atmospheric probe, would be needed to provide answers to these important questions.

2. In January 1983 the report of NASA's Solar System Exploration Committee (SSEC) was completed. In the discussion of the initial core missions, the SSEC executive summary contained a specific suggestion for a combined international Titan Probe and Saturn Orbiter mission, as follows:

The Titan Probe/Radar Mapper mission uses a modified Galileo probe together with a flyby or orbiter spacecraft equipped with a simple radar. The largest satellite of Saturn, Titan, is unique in having a thick atmosphere made up mostly of nitrogen, smaller but significant amounts of methane, and possibly argon. It is the only other atmosphere in the solar system that may be similar to Earth's before life arose. Atmospheric aerosols, believed to be organic compounds, obscure Titan's surface. The Core mission would determine the fundamental chemical composition of Titan's atmosphere and the nature of its unseen surface. The mission objectives could be achieved simultaneously with those of a Saturn Orbiter mission by the combination of a Galileo orbiter spacecraft in conjunction with a Titan probe supplied by an international partner. Such a mission could be launched before the end of the decade. 
3. Early in 1983 it became clear that NASA did not intend to go ahead with a U.S.-only mission to the Saturn-Titan system using spare Galileo-Jupiter subsystems and science investigations. However, much of a second Galileo spacecraft was already available, and preliminary studies showed that it could provide an exceptional platform for the orbiter part of the Cassini mission. For these reasons, the SSEC recommended that

Resources be made available in FY 1985 to preserve the option of building a spare Galileo orbiter to use for a Saturn orbiter mission, either alone or as part of an international collaborative project that also would send a probe into the atmosphere of Titan.

With this background in mind, the JWG instructed the Outer Planets Study Team to

...construct plans for candidate Saturn systems missions to be carried out jointly by the European Space Agency and National Aeronautics and Space Administration,

and suggested a study team report that would address the following points:

- statement of the scientific objectives to be addressed by each candidate mission and program,

- model payloads and rough mission concepts for each candidate mission,

- state of readiness of the required instrumentation and other technological project elements,

- special considerations, such as launch opportunities, and

- estimates of the costs and schedule.

The Outer Planets Study Team considered a large number of mission concepts based on use of different orbiter spacecraft (Galileo itself or a Mariner Mark II-class orbiter or an International Solar Polar Mission (ISPM)-class orbiter), different probe designs (a derivative of the Galileo-Jupiter probe or a new lightweight probe designed specifically for Titan), different launch vehicles (Shuttle-Centaur or Ariane), different interplanetary trajectories (direct or by Venus-Earth-Gravity Assist ( $\Delta$ VEGA)), and single or multiple launches (the latter implies use of an ISPM-class carrier for the Titan probe). In all cases, the study team considered a new Titan-Saturn science payload essentially derived from the discussions in the Cassini proposal, although several recently proposed scientific concepts (such as the possibility of ethane oceans on Titan) were also integrated into the assessments. 


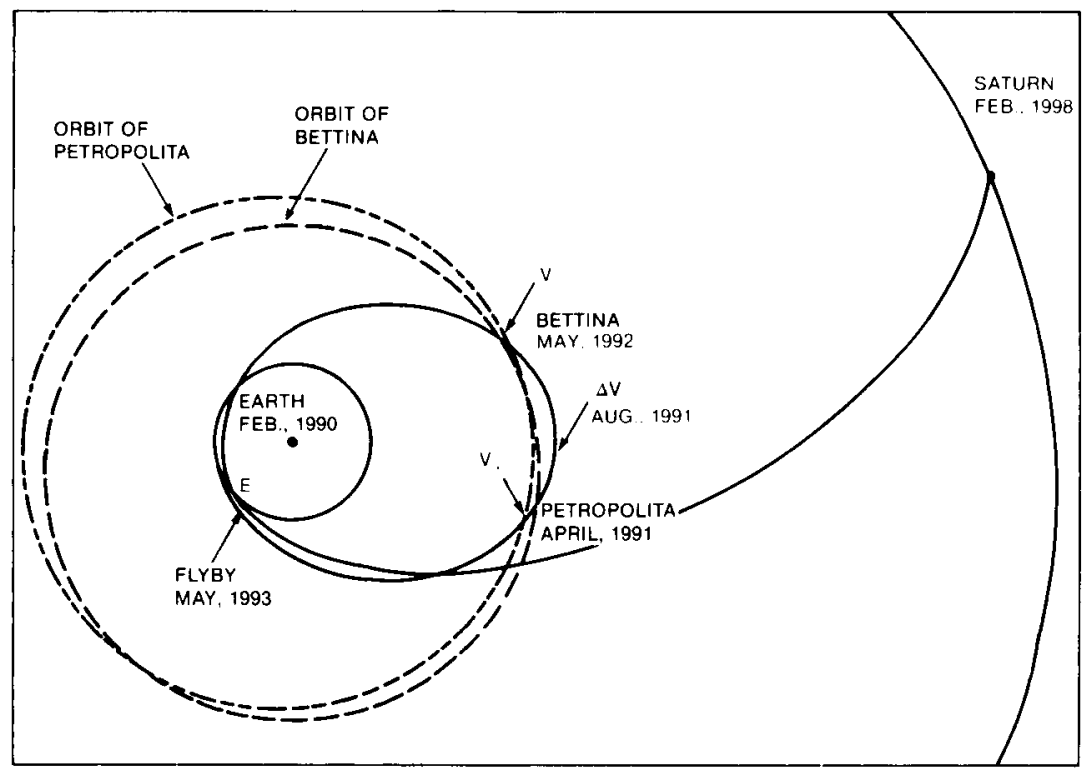

FIGURE B.1 The example shown is for a 1990 launch, but similar trajectories with asteroid flybys are found for launches in 1991 and 1992.

The study team concluded that, from the point of view of science return and cost effectiveness, the highest priority is given to a single-launch orbiter-probe $\triangle$ VEGA mission that uses a Galileo-class spacecraft and the new lightweight Titan probe. For programmatic reasons, the study team found it necessary to focus attention on launch opportunities in the early nineties, and Figure B.1 shows, as an example, the trajectory for a 1990 launch. Very similar trajectories are applicable for 1991 and 1992 launches. The long trip time associated with a $\triangle$ VEGA mission means that the orbiter-probe arrives at Saturn 8 years later, but Figure B.1 shows characteristic asteroid flyby opportunities that can yield early science returns of significance.

The unique advantages of the recommended highest priority combination arise from (1) the exceptional capabilities of the Galileo spacecraft, (2) the extensive altitude coverage at Titan associated with the lightweight probe, (3) the enhanced SaturnTitan science return from an orbiter-probe combination, and (4) the low mission cost associated with use of an existing spacecraft. 


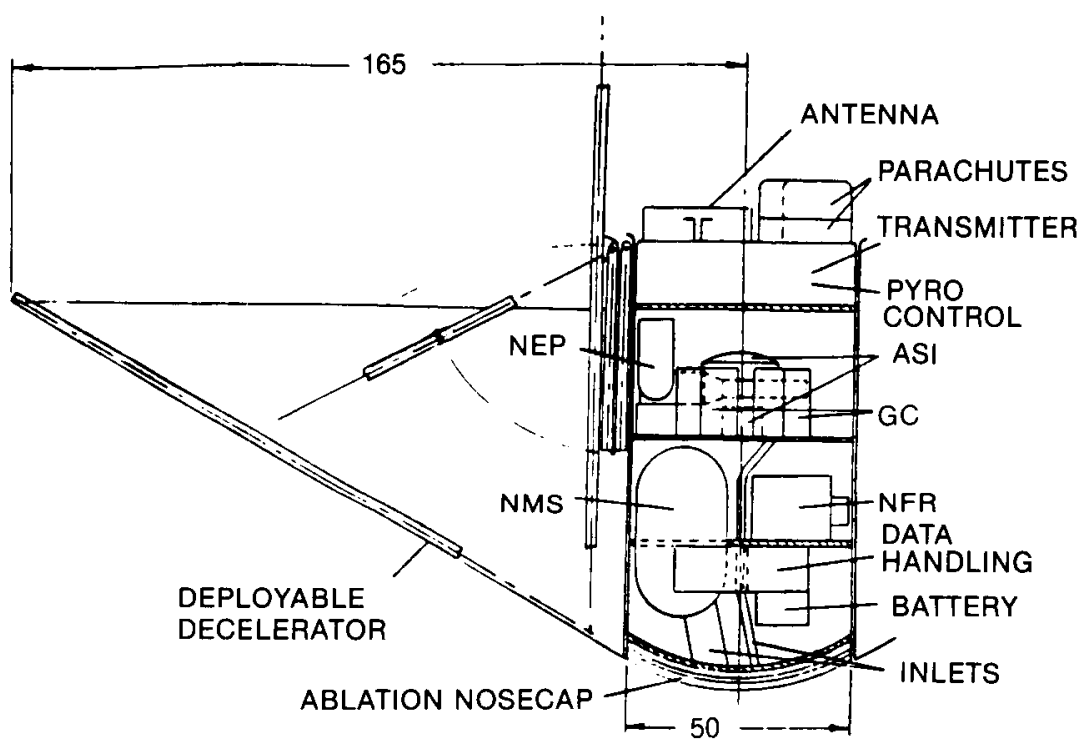

DESCENT SEQUENCE

DESCENT PROFILE
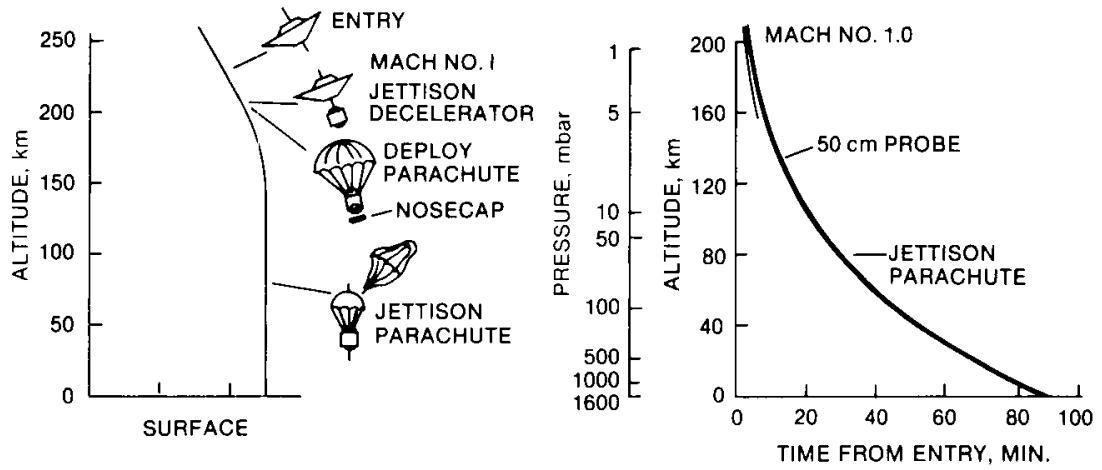

FIGURE B.2 (Top) Titan probe design. (Bottom) Descent sequence and profile.

These advantages are enumerated in somewhat more detail, as follows:

1. A Galileo-class spacecraft has dual-spin design, and particle investigators can obtain complete angular distributions, while imaging instruments and spectrometers can be precisely pointed. The Galileo imaging system has very high resolution, with image motion compensation and high data rate capability. There is a 
large-capacity tape recorder, a flexible command capability, a distributed data system, and a capability to carry the entire Cassini payload, including a Titan radar mapper (see section on model payloads, below). The spacecraft is designed to carry and deploy a probe, it has a probe relay antenna with precise pointing system and probe relay link, and it also has an orbit injection motor, plus an orbital maneuvering system with a large propellant capability.

2. The new lightweight Titan probe can provide continuous detailed measurements from the haze layer to the surface over a period of almost 2 hours (see Figure B.2). It has the ability to carry a comprehensive payload with landing science, and there are opportunities to include surface science investigations and optional balloon packages.

3. The scientific advantages of a combined Saturn Orbiter and Titan Probe mission are so great that they outweigh the disadvantages of the long trip time. Figure B.3 illustrates a possible Saturn tour and shows clearly that the orbiter can provide unparalleled opportunities to image Saturn and its ring system from high latitudes, to explore Saturn's magnetosphere, and to investigate the small icy satellites. In addition, Figure B.3 shows that the tour offers repeated close flybys of Titan, an opportunity that can provide invaluable information on Titan-plasma interaction and the Titan atmosphere (from radio occultation and remote sensing). In addition, the repeated Titan encounters will allow the radar mapper to build up an excellent surface image. The bottom part of Figure B.4 shows the ground tracks for the first eight Titan flybys (in the tour of Figure B.3), and the top part of Figure B.4 shows mapping coverage for just a small part (about one third) of the ground track for the initial flyby. 


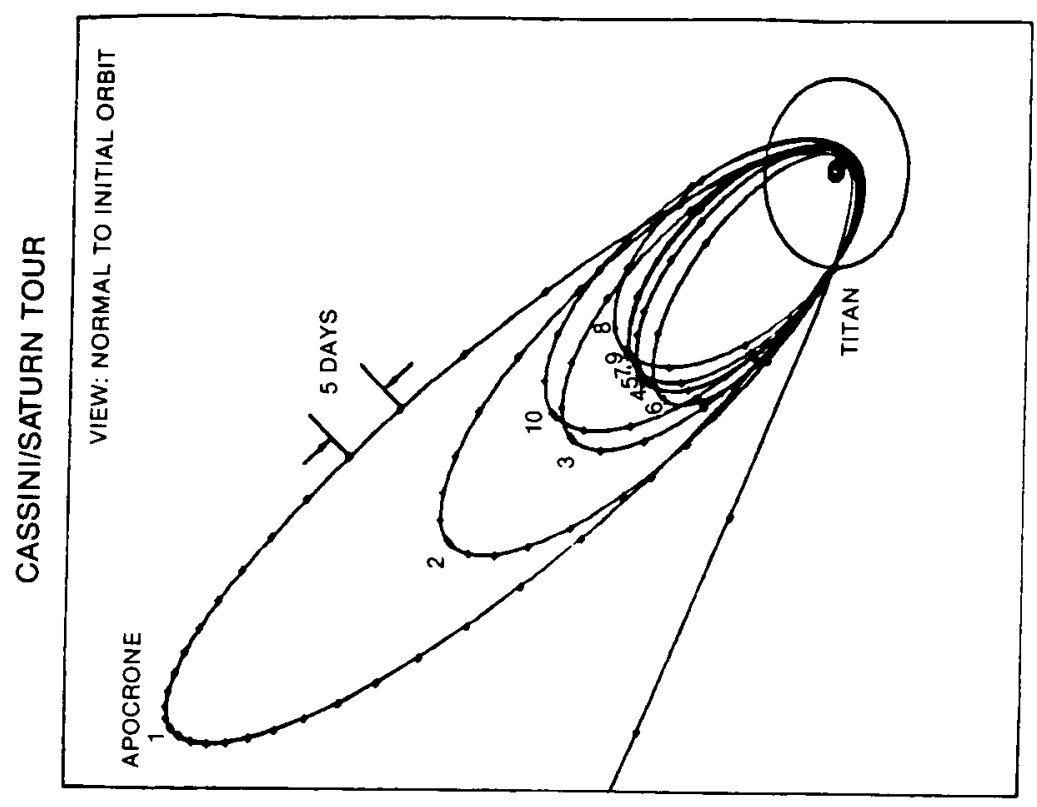

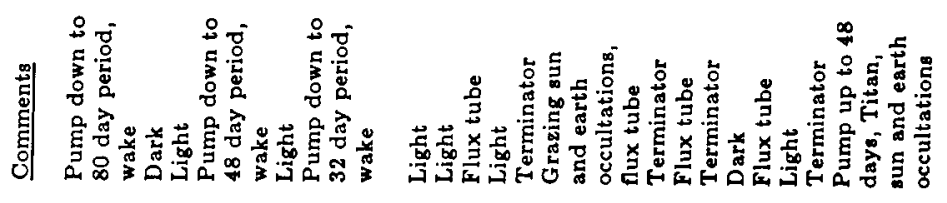

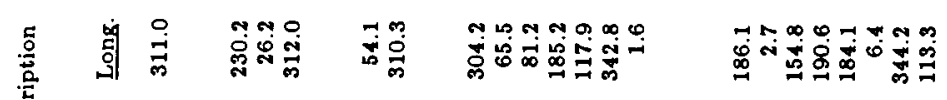

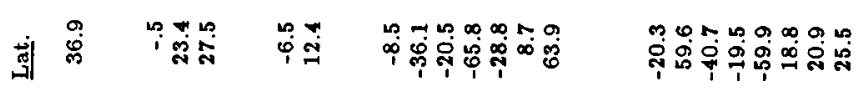

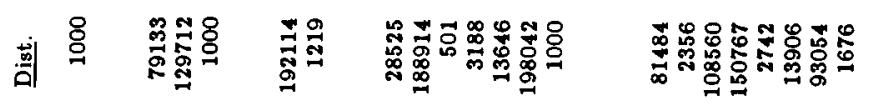

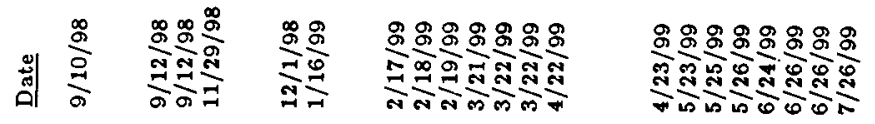

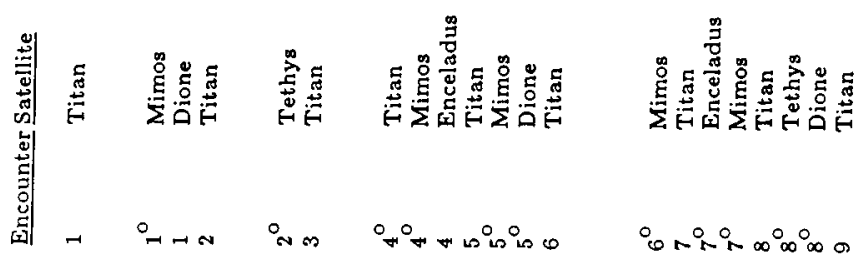


4. The cost advantage associated with use of an existing spacecraft is also impressive. An analysis by JPL of actual development costs for the Viking Orbiter, Voyager, and the Galileo Orbiter is contained in the back-up section below. JPL suggests that it would cost approximately 3 to 3.5 times as much to develop a new system with comparable capabilities. 
SAMPLE COVERAGE FOR FLYBY 1

$(-14$ to $-6.5 \mathrm{~min})$
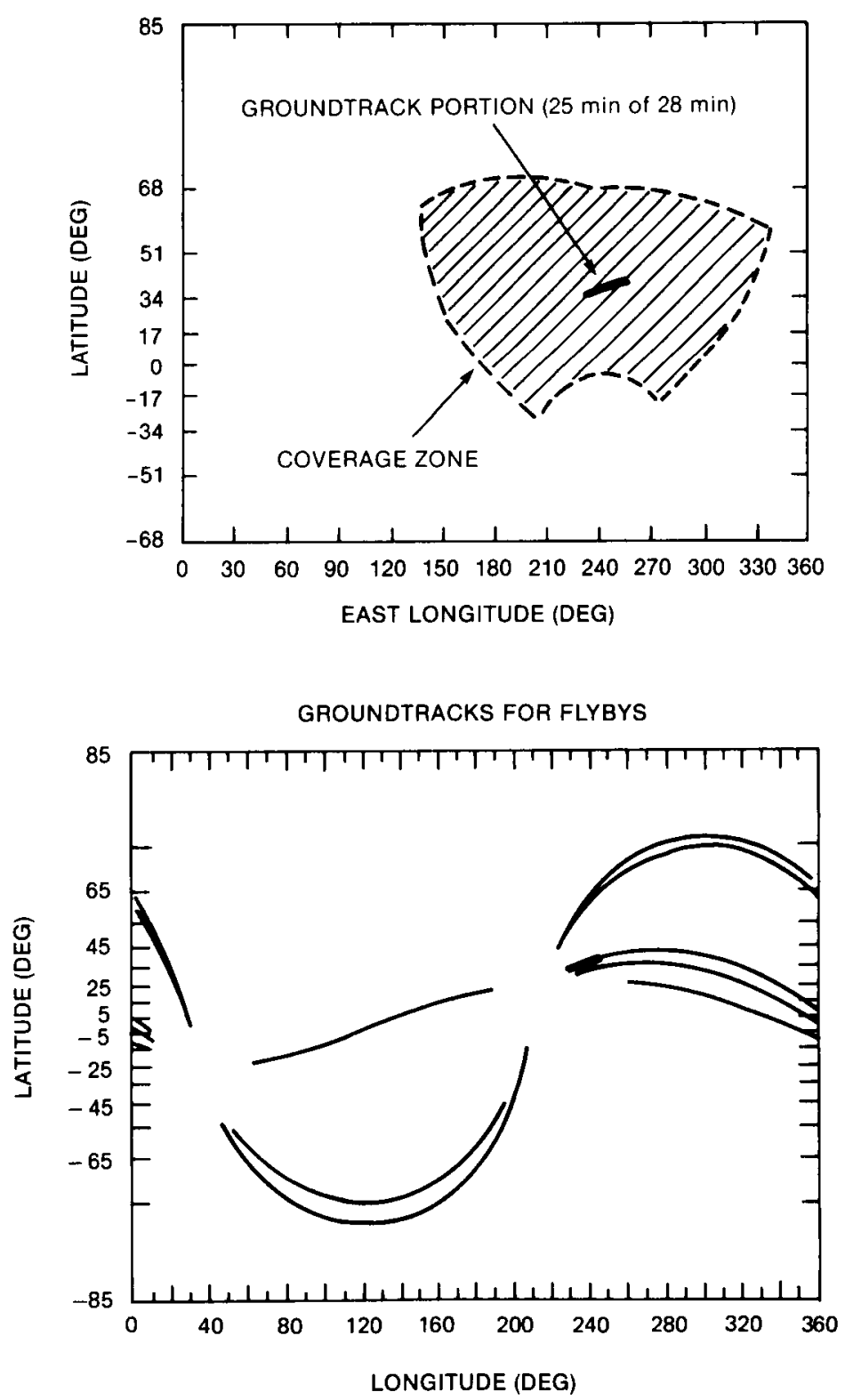

FIGURE B.4 Titan surface mapping. (Top) Portion of first pass. (Bottom) Ground tracks for eight passes. 


\section{SCIENCE OBJECTIVES}

\section{Saturn: Interior and Atmosphere}

\section{The Interior}

Science does not now have a firm knowledge of the interior of Saturn, but results from Voyager and theoretical considerations strongly suggest that it is qualitatively different from the interior of Jupiter. It is likely that at the high pressure levels occurring within Saturn, helium is differentiated from metallic hydrogen, leading to the formation of helium droplets that migrate toward the center of the planet, liberating gravitational energy. That may explain the observed depletion of helium in the outer atmosphere of Saturn compared with abundances in the sun and Jupiter.

The precise determination of the internal structure requires several new measurements. The celestial mechanics experiments from a Saturn orbiter would yield accurate values of the moments of the gravitational field, which provide knowledge of density distribution within the planet. Additional information might be obtained by the study of the resonant structure in the ring system. The determination of the temperature at the top of the convective region can provide key information on the spatial distribution of the internal sources (which probably counterbalances the seasonal variation of the solar insolation).

\section{Thermal Structure and Composition}

Voyager measurements suggest that a strong seasonal temperature effect does exist in the upper troposphere of Saturn. Far-infrared measurements from a Saturn orbiter would permit scientists to infer the thermal structure at all latitudes, including north and south poles, down to the 1-bar pressure level. Once the temperature profile is determined, the distribution of $\mathrm{NH}_{3}$ and of $\mathrm{PH}_{3}$ (the latter is a tracer of convective motion) could also be ascertained. The $\mathrm{H}_{2} / \mathrm{He}$ mixing ratio could then be determined more accurately than was possible from Voyager, and the abundance of various other minor components, such as $\mathrm{CO}$, 
could be also determined from the record of the far-infrared and submillimeter spectrum. The aeronomy of the Saturn atmosphere and its spatial variations could also be inferred from spectroscopic observation of various hydrocarbons known to be present in the stratosphere of the planet. An important issue is the understanding of the vertical structure and composition of clouds. Another outstanding problem is posed by the abundances of $\mathrm{CO}, \mathrm{GeHy}$, and $\mathrm{H}_{2} \mathrm{O}$, all of which have been detected in Jupiter's atmosphere but not yet on Saturn. These gases are tracers of atmospheric chemistry and vertical transport as well as potential clues to the overall composition of the planet.

Atmosphere Dynamics and General Circulation

The cause of the general circulation of the Saturn atmosphere is uncertain. One group of theories suggests that basic features of the unique wind pattern are generated by meteorological processes extending in depth to no more than a few thousand kilometers. A second group of theories assumes, on the contrary, that the observed pattern is the manifestation of the internal configuration of Saturn. Discrimination between various theories can be based on studies of the winds at very high latitudes; these winds can only be viewed from a highly inclined orbit, and such information was not obtained from Voyager. Now that it has been determined that Saturn electrostatic discharges (SEDs) come from atmospheric lightning associated with a huge equatorial storm center, scientists can look forward to using orbiter wave measurements to provide important new information on temporal variations in Saturnian meteorology.

In addition, Saturn orbiter observations will address the following important meteorological questions:

- What is the structure of motion at high latitude?

- What is the zonal motion profile? Is it symmetric?

- What is the magnitude of the eddy momentum transfer? How does it compare with Jupiter?

- What is the role of baroclinic waves at mid-latitudes in the heat transfer? 


\section{Titan}

Although the recent Voyager encounters have given major new insights into the structure and composition of Titan's atmosphere, Titan remains an essentially unexplored planetary body that poses many intriguing questions. These questions may be broadly classified as related to the nature of the surface (solid or liquid) and interior, the evolution of the atmosphere, Titan's organic chemistry in the atmosphere and possible connection with prebiological processes, and interaction with Saturn's magnetosphere. A crucial reason for determining the surface physical state is that a hydrocarbon ocean would exert a strong control on the atmospheric opacity structure, both in terms of producing haze or clouds as well as supplying gaseous methane as a photochemical and opacity source. The photochemical products would form an ocean, and the ocean itself could then be a determining factor in the state and evolution of the atmosphere. This is a strong incentive for including enough landed or surface science to answer once and for all (in conjunction with global radar) whether a widespread ocean exists.

The available data are consistent with the assumption that Titan's atmosphere is of secondary origin, either the product of photolysis of outgassed $\mathrm{NH}_{3}$ or evaporation of $\mathrm{N}_{2}, \mathrm{Ar}$, and $\mathrm{CO}$ from a clathrate hydrate. A measurement of the primordial ${ }^{36} \mathrm{Ar}$ to $\mathrm{N}_{2}$ ratio in the atmosphere can discriminate between these two evolutionary scenarios. Isotopic ratios and other noble gas abundance measurements can provide further constraints.

Titan's unique atmosphere is particularly conducive to continued chemical evolution. With an extended atmosphere, weak gravitational acceleration, and "warm" thermosphere, hydrogen in atomic and molecular form escapes efficiently from the exobase by thermal processes to ensure that $\mathrm{CH}_{4}$ photolysis produces a suite of complex heavy hydrocarbons whose ultimate fate is to accumulate on the "surface" and produce an ocean composed mostly of $\mathrm{C}_{2} \mathrm{H}_{6}$ with dissolved $\mathrm{CH}_{4}, \mathrm{~N}_{2}$, and other trace species. The determination of the existence of an ocean, its composition, and the morphology of any solid surface is of highest priority. Although the measured density indicates that Titan consists of about 50 percent rock and 50 percent ices, the internal structure of the satellite remains unknown, as does its rotational period. 
The Voyager 1 close encounter with Titan has shown Titan's interaction with the magnetosphere to be an important energy source to drive the photochemistry of neutral and ionized species and produce nonthermal escape of nitrogen, which is important to overall mass balance and the long-term evolution of Titan's atmosphere. In turn, Titan may be an important direct source of magnetospheric plasma by ion escape. At times of stronger solar wind pressure, the dayside magnetopause may move inside of Titan's orbit and fully expose Titan to direct solar wind interaction. The relative importance of these two types of interactions needs to be determined; it may also influence the course of chemical evolution. The thermal escape of $\mathrm{H}$ and $\mathrm{H}_{2}$ and nonthermal escape of $\mathrm{N}$ lead to the formation of tori, of which only $\mathrm{H}$ has been detected by Voyager. Since subsequent ionization of these neutrals by electron impact and charge exchange is an important plasma and energy source for Saturn's magnetosphere, measurements of the $\mathrm{H}_{2}$ and $\mathrm{N}$ tori are important objectives.

The interesting organic chemistry on Titan, which is driven by precipitation of magnetospheric electrons, solar UV radiation, and cosmic ray ionization, eventually leads to the formation of an extensive haze layer that, at the time of the Voyager 1 encounter, was asymmetric and darker in the north with a dark polar collar. Is this a permanent feature, or does it undergo seasonal and other variations? These aerosols, presumably condensed hydrocarbons, strongly absorb UV and visible solar radiation to drive Titan's atmospheric circulation. The optical properties of these aerosols, the differential heating rate distribution, and the global thermal signature need to be measured to adequately understand the general circulation of Titan's atmosphere.

Finally, Titan's reducing atmosphere could be similar to the mildly reducing prebiotic atmosphere of the Earth. The detection of prebiotic molecules ( $\mathrm{HCN}, \mathrm{HC}_{3} \mathrm{~N}$, and $\mathrm{C}_{2} \mathrm{~N}_{2}$ ) suggests that Ti$\tan$ would provide a unique laboratory to test theories of chemical evolution of organic molecules, which on the Earth, have led to the formation of life. In this context, it is necessary to search for additional complex organic molecules, identify aerosols, and evaluate the role of atmospheric electrostatic discharges. 


\section{The Rings}

Since 1610, Saturn's rings have fascinated astronomers and physicists, and analysis of the observations has remained a very active subject of research. Voyager measurements have greatly increased our appreciation of the complexity of this system. Collisions between particles, complex gravitational interactions between ring particles, satellites, and Saturn, and physical interactions between Saturn's magnetosphere, dust, and rings make the ring system a particularly fascinating laboratory of physics. Saturn's rings are not only interesting in themselves as the place of many phenomena not yet understood, but they are characteristic of many other flat disks in the Universe that are not easy to observe because they are too far from Earth in time as well as distance. Ring studies will provide information about the formation of the primitive solar nebula, the dynamics of flat galaxies, and the evolution of accretion disks around compact stars. The discovery of rings around Uranus and Jupiter, together with the astonishing structures observed by Voyager spacecraft around Saturn, can be considered as a major scientific event of the second half of this century. Observations of Saturn's rings by Voyager 1 were so surprising that the highest priority was devoted to the rings by the scientific teams for the Voyager 2 sequence of observations. Saturn's rings are so complex and exhibit so many basic physical mechanisms that further study should be considered one of the highest priorities of space exploration.

A number of highly significant questions remain open: What is the behavior of a particle, or rather a group of particles, inside the Roche limit as a function of the distance to the planet? How does accretion work inside the Roche limit? Do "particles" exist a long time, or are they continuously formed and destroyed? What is the size and mass distribution inside the Roche limit? Do "satellites" survive inside rings? Are there large clumps? What is the nature and the chemical composition of the particles? Is it possible to explain color differences observed by Voyager spacecraft as a result of differences in shape and size or of dynamical, electrical, or optical properties of the particles? Are the rings still evolving? What are the ring interactions with the environment (magnetosphere, micrometeorites, satellites, radio-electric sources, etc.)? Why are the rings not homogeneous? Why do they show a large number of 
complex structures such as narrow ringlets with sharp edges, irregular and kinky ringlets, wavy structures, eccentric rings? What is the cause for and the evolution of the nearly radial spokes in the $B$ ring? What is the source for the diffuse outer $G$ and $E$ rings? How do micron-sized particles interact with the magnetospheric plasmas and fields? What role do interplanetary meteoroids play for the destruction and erosion of large ring particles and for the generation of small particles?

A better understanding of the physics of the rings obviously requires more observations. After the Voyager encounters, any observation of Saturn's rings by a spacecraft should satisfy at least the following requirements:

- the highest possible resolution,

- observations with a large variety of phase angles,

- continuous observations of selected areas in order to follow their evolution with time,

- Occultation experiments (including stellar occultations observed in visible and ultraviolet as well as radio occultations), and

- Observation of small-particle phenomena.

The anticipated science return associated with these requirements can be described in the following:

High Resolution Voyager spacecraft revealed thousands of dark and bright divisions, as well as unexpected structures (eccentric rings, narrow rings with sharp edges, twisted rings, irregular rings, spiral waves, bending waves, etc.). For each value of the resolution on Voyager images, a new structure is visible. These rings have structure in which each detail observed at a better resolution divides itself in a set of smaller structures. There exist particles of every size from micron sized to kilometer sized. The ideal resolution would be the one allowing the observation of individual particles, as well as the possibility to follow their evolution with time. A resolution of the order of few hundred meters or 1 kilometer should be already particularly interesting; this should allow the possibility of observing the largest condensations of matter as well as small embedded satellites. Any resolution better than 1 kilometer should lead to the discovery of new rings, new waves, and new dynamical phenomena. A resolution of few hundred meters should allow scientists to follow the evolution of few large particles, to measure the global thickness of the rings, and to observe 
in detail spiral waves and bending waves. A resolution of several tens of meters should allow the observation of the local thickness of the rings (which is directly related to their dynamics) and the behavior of sharp edges (which is related to the presence of small satellites, resonances, and collisions).

Phase Angles Detailed observations of the same place in the rings observed under different phase angles give the respective amount of reflected, diffused, and transmitted light, and thus give the size distribution and some information on the surface properties of the particles as a function of the distance to the planet.

Time Evolution In addition to the evolution of spokes, it seems that some parts of the rings evolve on a short time scale like the atmosphere of a planet that is a dynamically "active" object. The direct observation of such an evolution should allow, for the first time, the direct measurement of the exchange of energy and momentum inside the rings and thus provide a better understanding of complex phenomena such as resonances and collisions. The continuous observation of a selected area is thus one of the major priorities.

Occultations Observation of star occultations by rings is a powerful tool to obtain the highest possible resolution on rings. An orbiter around Saturn should allow the observation of several occultations per revolution. A comparative study of the results of radio occultation, star occultations, and direct observations under different phase angles should give a good "map" of the rings with particle size distribution, nature of the particles, and dynamical evolution as a function of the distance to the planet.

Small-Particle Phenomena Voyager observations showed not only that there are small (micro-sized) particles in the Saturnian ring system, but that they play an important role in many ring-, satellite-, and magnetosphere-related phenomena. The Saturnian magnetosphere is a dusty magnetosphere, where a large number of phenomena can be studied that are of utmost significance during the formation of planetary systems and in other astrophysically important dusty plasmas. An in situ dust analyzer will be especially helpful in providing new input and data not otherwise obtainable. In near-polar orbits, a global view of the ring system will be possible. This information, together with edge-on views 
during ring-plane crossing, will set important constraints on modeling of the three-dimensional structure of spokes and will help in understanding the physical processes that lead to their formation and evolution.

Ground-based and space observations detected diffuse outer rings that consist mainly of micron-sized particles. Plasma and energetic particle absorption features indicate even more extended rings. The origin of these ring particles and their dynamics are not understood. Observations by a variety of methods (optical imaging of the large-scale distribution, in situ measurements of the local spatial density and size distribution, high-energy particle absorption measurements of the mass density) are needed together with measurements of the dynamical state of these particles (trajectories, speeds, and charge state) as well as the ambient magnetospheric conditions (plasma energy, density, and composition and magnetic field properties).

The characterization of the interplanetary meteoroid flux at Saturn will aid in understanding the impact of these particles on the satellites and rings i.e., the redistribution of the particulates in the rings, the formation of the neutral ring atmosphere, and the injection of heavy ions from the rings.

\section{Icy Satellites}

The images returned from Voyager 1 and 2 have shown that the Saturn satellites (other than Titan) are icy objects, intermediate in size between asteroids and Galilean satellites, and that they have surfaces that are not uniformly cratered and, hence, not of the same age. After their formation, the satellites of Saturn, in spite of their small mass and size, appear to have significantly evolved, and consequently, their surface ages and tectonic structures are different. Moreover, the duration and the degree of evolution for each satellite seem to be different. In contrast with the Galilean satellites of Jupiter, the Saturnian satellites also display a number of orbital combinations that might indicate distinctly different evolutionary histories of these two satellite systems. Presently, there are 17 satellites with well-determined orbits in the Saturn system, and it is possible that several small objects still remain to be discovered.

Several important improvements will be achieved by a Saturn orbiter mission: 
1. A systematic search could be carried out to look for objects like the Mimas companion indicated in the charged-particle observations of Pioneer 11 and Voyager.

2. A more accurate determination of the densities is needed so that interior models and models of thermal evolution will be better constrained. Scientists also need to know how the densities vary with radial distance. In fact, the Saturnian satellites have densities consistent with a mixture of ices and silicate particles, and thus models of thermal evolution are based on chondritic abundances of long-lived radionuclides in the silicate component of the mixture.

3. It will be possible to produce imaging maps that are much more complete than the results obtained by Voyager 1 and 2 . The delineation of the surface morphology and structure of these satellites will be aided considerably by the operation of radar mapper during the close encounter. More detailed imaging of the surface features will establish whether the first-order tectonics of these bodies has been compressional or extensional, giving new geological information to thermal modelists. Moreover, close approach not only with the major satellites but also with some (or all) of the Lagrangian satellites of Tethys and Dione should allow crater counting that, compared with data coming from the other satellites, might give a relative chronology of the disruption and reaccretion events in the inner Saturn system.

4. The family of larger satellites should be studied not only from the geomorphological and geophysical points of view, but also from the point of view of surface processes related to their magnetospheric interaction. For example, sputtering by energetic charged particles and micrometeoroids and electrostatic charging of the satellite surfaces are interesting issues characteristic of the magnetospheric environment of Saturn. Voyager observations have suggested that Dione might be a source of magnetospheric plasma, and a detailed survey of its surface could reveal how the gas might be emitted.

5. The importance of Enceladus in controlling the Saturnian magnetosphere can be verified. This satellite can be the source of the tenuous $\mathrm{E}$ ring. In fact, ground-based observations have shown that the brightness of the $E$ ring peaks in the vicinity of Enceladus. High-resolution imaging of Enceladus is needed. The several distinct terrains on this body, with widely differing ages based on cratering statistics, must be imaged at the highest possible resolution, and in particular, the boundaries between the 
regions have to be studied. This may yield clues to the history and mode of resurfacing of the body and, thus, perhaps to the mechanisms (e.g., tidal?) for the generation of its thermal activity. Although high-resolution imaging must be achieved for the icy satellites in general, Enceladus is sufficiently special and intriguing that it makes a strong case by itself for having Galileo-quality imaging on board a Saturn orbiter.

6. Iapetus is also an intriguing special case for which good remote sensing can provide new information on the composition of the dark hemisphere and the origin of this dark material.

\section{Magnetosphere}

A Saturn orbiter with repeated Titan encounters will open up a whole range of opportunities to answer questions that were, at best, only posed by the Pioneer and Voyager missions. Thus far, even such basic questions on plasma composition, torus composition, and dominant convection mechanism in the Saturn magnetosphere are open. Moreover, this will provide the only opportunity to have high-latitude in situ measurements from a centrifugally dominated magnetosphere. In what follows, the study group concentrated on the scientific questions that will be addressed by an orbiter mission.

Unlike the terrestrial magnetosphere, Saturn, like Jupiter, has major sources of plasma within the magnetosphere from satellitesmost notably, Titan and the icy satellites Tethys and Dione, and the rings. In addition, the magnetosphere itself, by providing a continual flux of particles and energy, has a significant role in satellite atmospheric and surface science. At Saturn, in situ composition measurements over a wide energy range are central to establish the relative importance of planetary, solar, and satellite ring sources, as well as to identify the plasma heating and redistribution mechanisms within the system. As at Jupiter, centrifugal effects are important at Saturn, but the magnetosphere convection may switch between a planetary wind and solar wind driven (terrestrial) mode. The magnetosphere is further challenging in that we know that plasma-dust and ring interactions act as sinks and sources of ionized material, although the processes are not quantitatively understood. Furthermore, there is a large neutral 
hydrogen torus extending from Titan's orbit into $\sim 8 \mathrm{R}_{S}$ radial distance with poorly understood charge-exchange interactions with the plasma environment.

Magnetospheres are recognized as sources of radio emissions, and in Saturn's case the emissions show very puzzling features that are unique to Saturn, as well as features similar to those found at other planets. At Saturn the kilometric emissions are strongly modulated by the solar wind, and the dayside cusp appears to be a strong source. However, in spite of the strong alignment of magnetic field and rotation axes, the Saturnian emissions exhibit a preferred longitude excitation region. Another very perplexing characteristic is related to the phase dependence; at Saturn the emissions turn on and off simultaneously all around the planet, while at Jupiter there is a beaming or "searchlight" effect.

Radio observations constitute one form of remote sensing. The $\mathrm{H}$ torus can also be remotely sensed in the UV. A further important form of remote sensing, for magnetospheric purposes, involves auroral observations that are of significance in assessing the relative importance of internal and externally driven convection, as well as the strength of particle loss and the energy input into the ionosphere.

Quantifying the plasma loss into the atmosphere from any magnetosphere requires a detailed knowledge of the plasma wave spectrum below the radio bands. Measurement of the spectrum is necessary for other reasons, for such waves can provide anomalous diffusion, resistivity, and other transport effects, as well as a local diagnostic of electron density at times achievable in no other way (cf. the Voyager encounter with the Titan wake).

The polar orbit required for the mission has distinct advantages for magnetospheric studies. The close alignment of Saturn field and rotation axes makes it hard to obtain latitude coverage of plasma distribution along the field in any low-inclination orbit. With a high-inclination orbit, it occurs naturally, with radial coverage being achieved by successive orbits. This will provide an excellent contrast with the Galileo Jovian magnetospheric data; polar and auroral field magnetic field lines may be directly explored.

The Saturn magnetosphere has associated with it a second magnetosphere-namely, Titan's-induced by the corotating Saturnian plasma flow or, at times, the exterior sheath flow. A bipolar tail is known to form, and resemblances to cometary interactions 
or Venus are clear. Since the orbiter will have repeated flybys of Titan, the opportunity exists for detailed investigation of the effect of the interaction in removal of atmospheric constituents from Titan's atmosphere. 


\section{MODEL PAYLOADS}

Model payloads that respond to the science objectives of the preceding section are given in Table B.1 for the orbiter and in Table B.3 for the probe. Table B.2 relates the orbiter instruments to the scientific questions that they will address. These payloads are capable of giving important insights into the outstanding scientific questions but should not be regarded as excluding other significant measurements.

The model payload for the orbiter is basically the same as the one given in the Cassini proposal. The main changes involve (1) retention of the present Galileo imaging system, (2) use of Pioneer Venus specifications for the Titan radar mapper, and (3) use of the names "energetic particle detector" and "plasma wave/radio emission detector" rather than "cosmic ray detector" and "plasma wave analyzer." The science objectives associated with the individual instruments are summarized in Table B.2.

The accommodation of this recommended payload on a Galileoclass spacecraft is addressed in Figure B.5. The top panel shows the spacecraft configuration with the present Galileo-Jupiter payload. The bottom part of the figure contains a table showing how the study group's recommended Saturn orbiter payload could be mounted on Galileo; all numbers in the right-hand column represent Galileo-Jupiter equivalents, and the other list is taken from Table B.1.

It can be seen that the recommended science payload for the Saturn orbiter is quite comparable to that of Galileo-Jupiter in terms of total mass, power requirement, and utilization of spinning and despun sections. The study group concluded that an orbiter spacecraft with significantly less capability than Galileo could not deliver the needed science return from Saturn.

The recommended instruments for the Titan probe are shown in Table B.3, and the science objectives are summarized in Table B.4. These probe investigations are primarily focused on in situ measurements of the atmosphere below $200 \mathrm{~km}$ but will also define the upper atmosphere, ionosphere, and aerosol composition above $200 \mathrm{~km}$. An important goal of the probe instruments is to determine the nature of the surface, liquid or solid, during the 
landing period, as well as the composition of the surface, should landing be survived for even a few minutes. (The possibility of landing survival at a touchdown velocity of $\sim 7$ meters per second appears real and can be enhanced by use of a landing-shock absorbing design.) A simple measurement of liquid density (should the surface be liquid ethane, for instance) will be an important input to defining liquid composition. Use of the mass spectrometer to perform more detailed and specific analyses of surface material is feasible, given a suitable, additional sample inlet system. Radar altimeter measurements from the orbiter or flyby bus also can help determine the liquid-solid nature of the surface. 
TABLE B.1 Model Payload for the Orbiter

\begin{tabular}{|c|c|c|c|c|}
\hline Instrument & $\begin{array}{l}\text { Mass } \\
(\mathbf{k g})\end{array}$ & $\begin{array}{l}\text { Avg. } \\
\text { Power } \\
\text { (W) }\end{array}$ & $\begin{array}{l}\text { Approx. Data Rate }{ }^{2} \\
\text { (bits/s) }\end{array}$ & Source \\
\hline Imaging camera & 28 & 24 & 37 Kbits/s & Galileo orbiter \\
\hline Radar mapper & 16 & 45 & $\begin{array}{l}100 \text { Mbits on board } \\
\text { storage per Titan } \\
\text { encounter and trans- } \\
\text { mission up to full } \\
\text { rate allowance }\end{array}$ & New/Pioneer Venus \\
\hline $\begin{array}{l}\text { Far infrared } \\
\text { spectrometer }\end{array}$ & 17 & 5 & 600 & New/Vega \\
\hline UV spectrometer & 8 & b & 300 & $\begin{array}{l}\text { Venera, Galileo } \\
\text { orbiter, Voyager, } \\
\text { Pioneer Venus }\end{array}$ \\
\hline Dust analyzer & 8 & 10 & 100 & $\begin{array}{l}\text { Giotto, Galileo } \\
\text { orbiter }\end{array}$ \\
\hline Neutral gas analyzer & 4 & 6 & 200 & Giotto/Vega \\
\hline Plasma analyzer & 6 & 5 & 200 & $\begin{array}{l}\text { Numerous European } \\
\text { missions (e.g., }\end{array}$ \\
\hline $\begin{array}{l}\text { Ion composition } \\
\text { analyzer }\end{array}$ & 5 & 5 & 200 & $\begin{array}{l}\text { Geos, ISEE, Helios) } \\
\text { and Galileo } \\
\text { Pioneer Venus, and }\end{array}$ \\
\hline $\begin{array}{l}\text { Energetic particle } \\
\text { detector }\end{array}$ & 4 & 3 & 100 & ISPM \\
\hline Magnetometer & 3 & 3 & 200 & ISPM, Galileo, etc. \\
\hline $\begin{array}{l}\text { Plasma wave/radio } \\
\text { emissions }\end{array}$ & 5 & 5 & $\begin{array}{l}300 \text { (plus high data } \\
\text { rate made for wave- } \\
\text { form measurements) }\end{array}$ & $\begin{array}{l}\text { Voyager, ISPM, } \\
\text { Galileo }\end{array}$ \\
\hline Total & 104 & $\begin{array}{l}71+ \\
45 \text { for radar }\end{array}$ & & \\
\hline
\end{tabular}

${ }^{\mathrm{a}}$ The orbiter data rate at Saturn is assumed to be $10 \mathrm{Kbits} / \mathrm{s}$. 


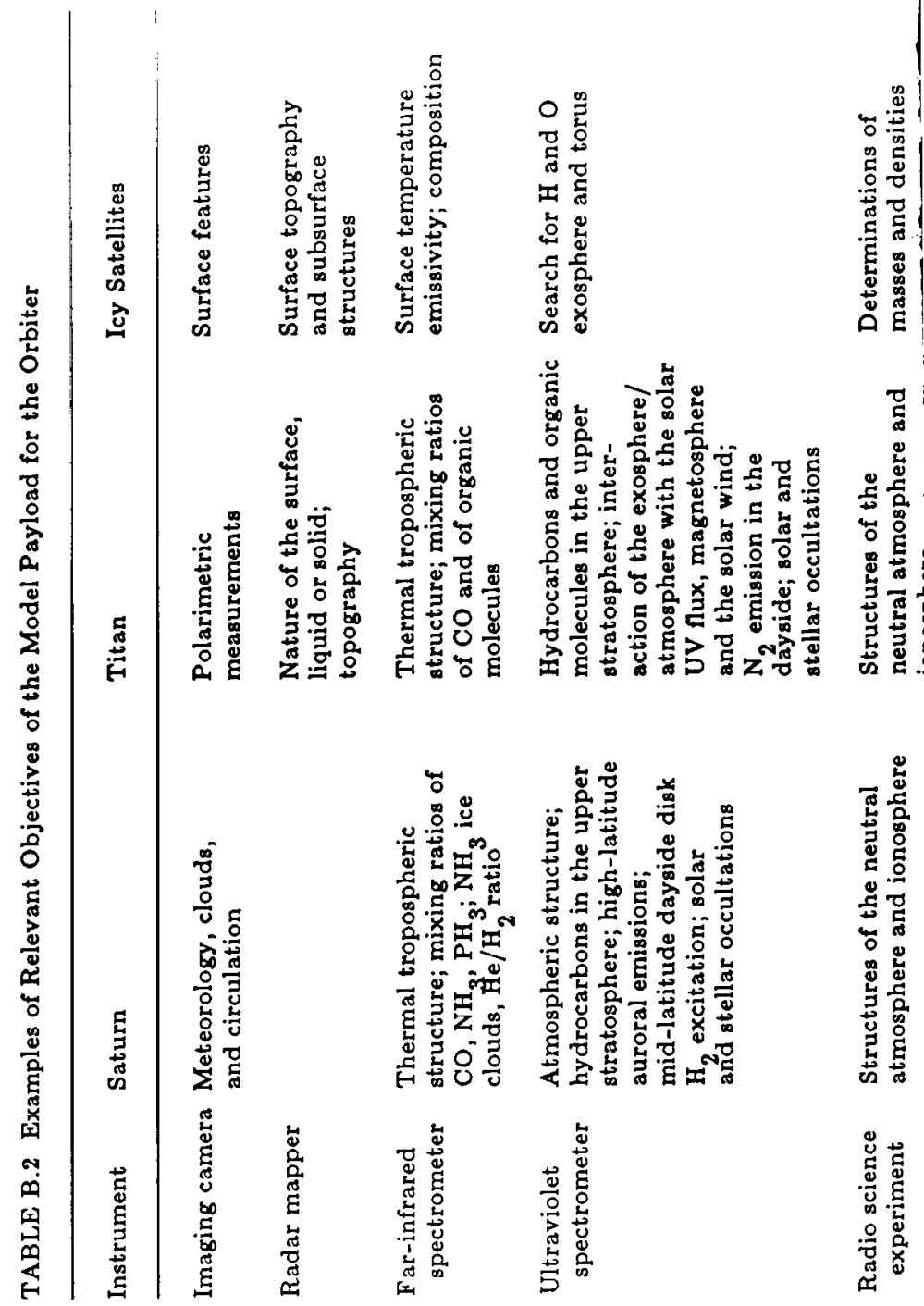



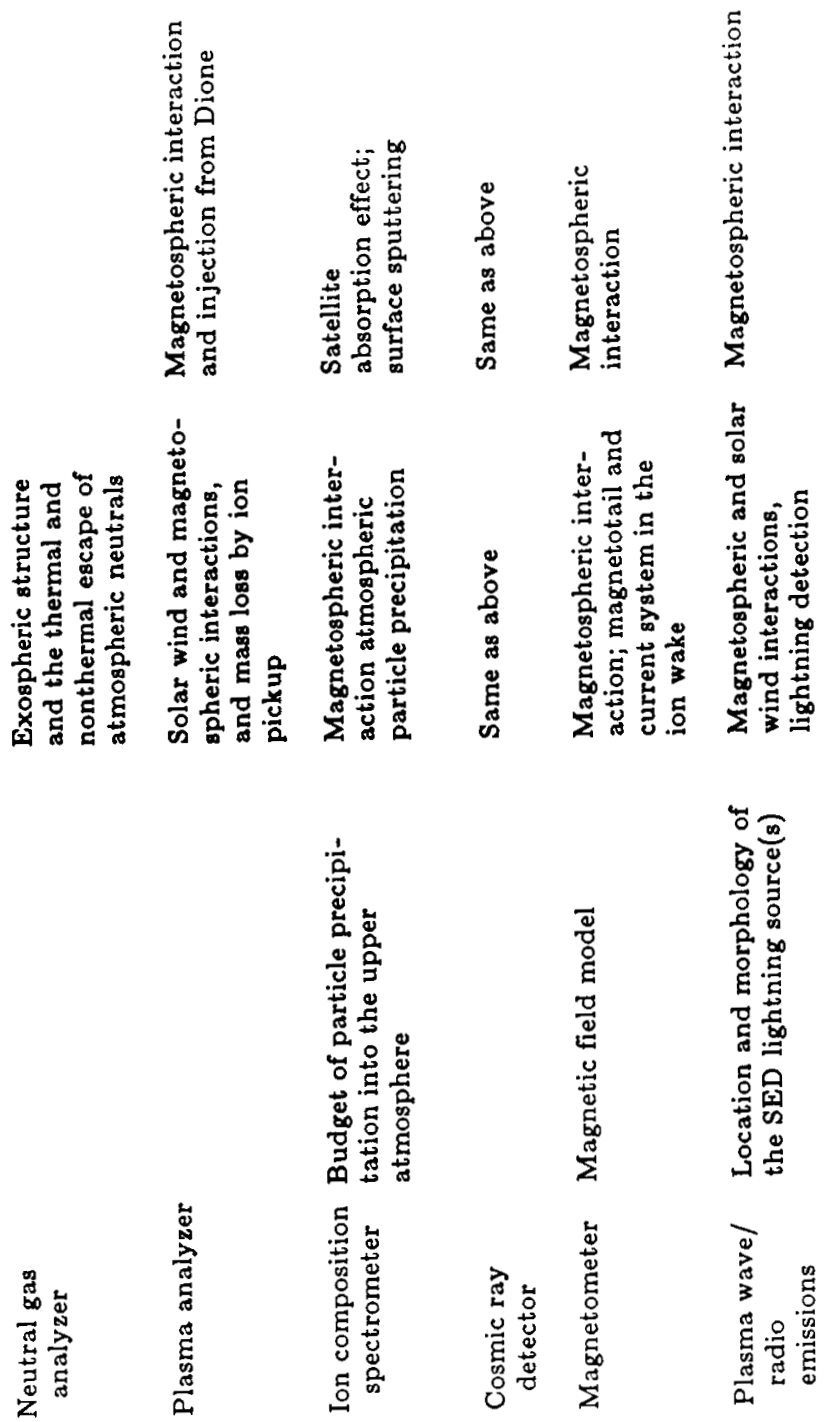


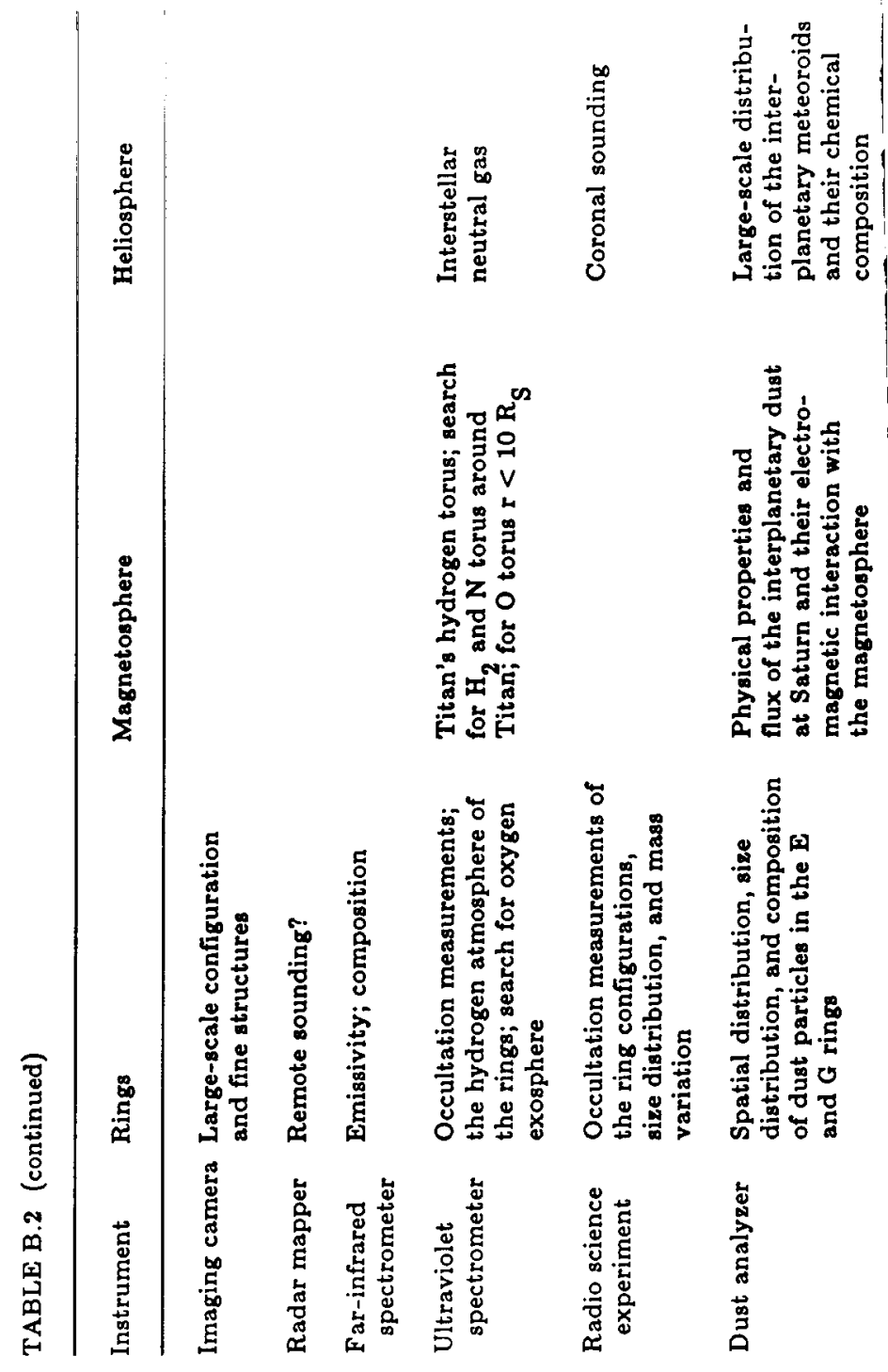


TABLE B.3 Titan Probe Payload

\begin{tabular}{lccll}
\hline Instrument & $\begin{array}{l}\text { Mass } \\
(\mathbf{k g})\end{array}$ & $\begin{array}{l}\text { Power } \\
\text { (W) }\end{array}$ & Heritage \\
\hline Neutral mass spectrometer (NMS) & 10 & 30 & Galileo, Giotto/Vega \\
Gas chromatograph & 4 & 20 & New design \\
Near-infrared spectrometer & 3 & 7 & Vega \\
$\begin{array}{l}\text { Atmosphere structure instrument } \\
\text { (ASI) }\end{array}$ & 4 & 6 & $\begin{array}{l}\text { Galileo, Pioneer Venus, } \\
\text { etc. }\end{array}$ \\
$\begin{array}{l}\text { Descent imager (net flux radiometer) } \\
\text { Nephelometer }\end{array}$ & 5 & 10 & New design (Galileo) \\
Electric discharge detector & 4 & 13 & Galileo, Pioneer Venus \\
Wind measurement (oscillator) & 2 & 2 & Galileo, ISPM \\
\hline
\end{tabular}

NOTE: Payload may also include, for landing science, an ASI accelerometer, a float depth indicator, a pressure sensor, NMS composition data, orbiter radar altimeter, etc.; for possible preentry science, an ion mass spectrometer and retarding potential analyzer; and an optional Titan balloon package. 


\section{GALILEO CONFIGURATION FOR JUPITER MISSION}

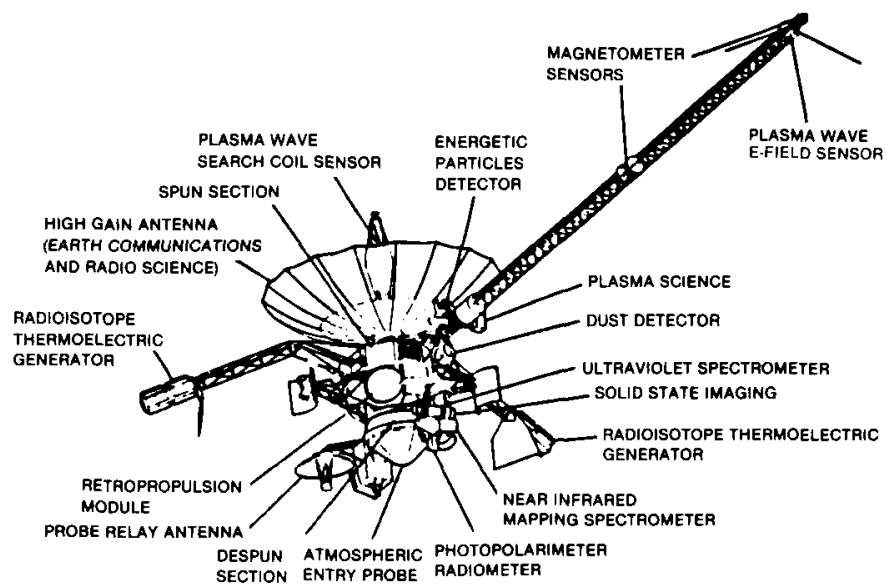

\begin{tabular}{|c|c|c|c|}
\hline \multicolumn{3}{|c|}{ SATURN ORBITER PAYLOAD ON GALILEO SPACECRAFT } & GALILEO-JUPITER PAYLOAD \\
\hline - DESPUN SECTION & $\begin{array}{l}\text { IMAGING } \\
\text { INFRARED SPECT } \\
\text { UV SPECT } \\
\text { PHOTOPOL } \\
\text { TITAN RADAR }\end{array}$ & $\begin{array}{r}28 \mathrm{~kg}, 28 \mathrm{w} \\
17 \mathrm{~kg}, 5 \mathrm{w} \\
8 \mathrm{~kg}, 5 \mathrm{w} \\
-16 \mathrm{~kg}, 45 \mathrm{w}\end{array}$ & $\begin{array}{rrrr}28 & \mathrm{~kg}, & 28 & \mathrm{w} \\
18.2 \mathrm{~kg}, & 15 & \mathrm{w} \\
4.2 \mathrm{~kg}, & 8 & \mathbf{w} \\
4.3 \mathrm{~kg}, & 11 & \mathrm{w} \\
- & -- & --\end{array}$ \\
\hline \multirow[t]{2}{*}{ - SPINNING SECTION } & $\begin{array}{l}\text { DUST ANALYZER } \\
\text { PLASMA ANALYZER } \\
\text { ION COMPOSITION } \\
\text { ENERGETIC PARTICLE } \\
\text { MAGNETOMETER } \\
\text { PLASMA WAVE/RADIO } \\
\text { EMISSION } \\
\text { NEUTRAL GAS }\end{array}$ & $\begin{array}{r}8 \mathrm{~kg}, 10 \mathrm{w} \\
11 \mathrm{~kg}, 10 \mathrm{w} \\
4 \mathrm{~kg}, 3 \mathrm{w} \\
3 \mathrm{~kg}, 3 \mathrm{w} \\
5 \mathrm{~kg}, 5 \mathrm{w} \\
4 \mathrm{~kg}, 6 \mathrm{w}\end{array}$ & 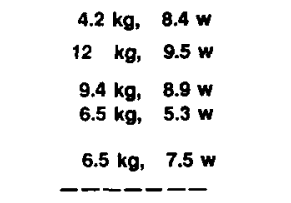 \\
\hline & & $104 \mathrm{~kg}, 71 \mathrm{w}$ & $93 \mathrm{~kg}, 102 \mathrm{w}$ \\
\hline
\end{tabular}

FIGURE B.5 Accommodation of recommended payload on a Galileo-class spacecraft. 
TABLE B.4 Titan Probe Science Objectives

Science Objective

1. Composition of the atmosphere

2. Thermal structure of the atmosphere

3. Cloud structure and physics

4. Aerosol composition

5. Nature and composition of surface

6. Lightning detection

7. Wind measurement

8. Upper atmosphere neutral composition and structure (Preentry package)

9. Ionosphere composition and structure magnetosphere-ionosphere interactions (Orbiter)
Relevant Instruments

Neutral mass spectrometer Gas chromatograph

Near-infrared spectrometer

Atmosphere structure instrument Net flux radiometer/imager

Nephelometer

Neutral mass spectrometer ${ }^{a}$ Gas chromatograph

Net flux radiometer/imager ${ }^{2}$ Landing science instruments: Atmosphere Structure Instrument (ASI) accelerator ${ }^{a}$

Float depth indicator, submerged pressure sensor, others

Surface composition

Neutral mass spectrometer ${ }^{a}$

Topography

Orbiter radar altimeter ${ }^{2}$

Electrostatic discharge detector

Stable oscillator

Neutral mass spectrometer

Ion mass spectrometer

Retarding potential analyzer

Electron temperature probe

Magnetometer

Plasma probe

Ion composition

Plasma wave

Energetic particle

${ }^{a}$ Multipurpose instrument. 


\section{STATE OF READINESS OF INSTRUMENTATION AND SUBSYSTEMS}

With the exception of a magnetometer/plasma wave boom and a redundant transmitter, all elements for a second Galileo spacecraft will be available (or could be made available) for use in a Saturn-Titan mission. Thus, the orbiter spacecraft itself, its subsystems, or its designs will essentially be in a state of readiness at any time following the 1986 launch of Galileo-Jupiter. The recommended orbiter science instruments are all largely based on existing designs, and it should be possible to assemble a payload at any time.

The Titan probe design is new, and some development is needed; but Titan entry conditions are similar to those encountered in the Earth's upper atmosphere, and it should be possible to verify the design with straightforward tests.

All of the recommended instruments for the probe exist in some form, but the miniaturized version of the gas chromatograph needs to be developed. 


\section{SPECIAL CONSIDERATIONS/LAUNCH OPPORTUNITIES}

The most significant "special consideration" involves the study group's recommendation that a Galileo-class spacecraft (more precisely, the second Galileo) be used for the Saturn orbiter. The study group concluded that a spacecraft with significantly less capability cannot deliver the necessary science return from Saturn, while a new orbiter spacecraft with equivalent capability would cost NASA so much that it would not provide a suitable basis for a cooperative ESA-NASA mission. These considerations involve designs, hardware, and personnel associated with the present Galileo-Jupiter mission, and the study group's recommendations imply that ESA and NASA agree to start the Titan Probe and Saturn Orbiter mission as the first of the joint planetary missions. 


\section{COSTS AND SCHEDULE}

The baseline recommendation of the Outer Planets Study Team involves use of the second Galileo spacecraft, and the study team therefore presents the costs and schedule in terms of this baseline option. (As shown in the following section, the orbiter costs would be scaled up by a factor of 3 to 3.5 if a new design were to be utilized.) The fundamental simplifying assumption for a Saturn-Titan launch in the early 1990 s involves a schedule that enables the Galileo-Jupiter flight team to participate in the Saturn orbiter integration during the Galileo-Jupiter cruise phase (mid1986 through mid-1988). This does not necessarily require an early U.S. new start for the Saturn-Titan mission itself, but it can require an early start (in the United States and in Europe) for the orbiter scientific instrument development; a reasonable schedule would have delivery of the Orbiter science instruments near the start of FY 1988.

In order to estimate the cost of the Orbiter section of the mission for a 1992 launch, JPL assumed the following:

- ESA supplies the probe, and Federal Republic of Germany supplies the retropropulsion module.

- Two RTGs can be procured from the Department of Energy for $\$ 20$ million (FY 1984 dollars).

- There will be no change to the basic Galileo spacecraft, and all orbiter instruments will be designed to meet existing Galileo interfaces.

- The development will be managed by the Galileo Project at JPL, and the mission/science will be constrained to fit.

- The mission will have a $3+\Delta$ VEGA trajectory with an 8-year trip time. tained.

- The Galileo solid state imaging (SSI) system will be re-

With these assumptions, the JPL estimate for a 1992 launch is approximately $\$ 170$ million, without mission operation and data analysis costs or science costs (except for SSI). 
TABLE B.5 Estimate of Total Costs in Millions of FY 1984 Dollars (1992 Launch)

\begin{tabular}{lcl}
\hline & Cost & Funding Agency \\
\hline Galileo spacecraft & 170 & United States \\
Titan probe & 83 & ESA \\
Shuttle-Centaur launch & 150 & United States \\
Operations & 150 & $\begin{array}{l}\text { United States and ESA; division } \\
\text { to be determined }\end{array}$ \\
Science & 50 & $\begin{array}{l}\text { United States } \\
\text { European member states }\end{array}$ \\
\hline
\end{tabular}

The probe development costs have been estimated independently by NASA/Ames Research Center and by Centre National d'Etudes Spatiales, and in both cases, the study group finds an estimate of about $\$ 83$ million. The study group's estimate of the total costs for both sides is shown in Table B.5. 


\title{
BACK-UP MATERIAL FOR GALILEO COST ESTIMATE
}

\author{
July 5,1983 \\ Refer to: 230-JRC:ps-026
}

Dr. Frederick L. Scarf

TRW Defense \& Space Systems

Building R-1, Room 1176

One Space Park

Redondo Beach, CA 90278

Dear Fred:

You asked me to provide an estimate of how cost effective the use of the Galileo orbiter for the Cassini mission would be compared to a new orbiter designed from scratch. In response I would say that at a cost of $\$ 150 M$ in FY84 $\$$, using the Galileo Orbiter would be a factor of 3 to $3-1 / 2$ times less costly than developing a new system.

The approach used in arriving at this estimate was to take the actual development costs for three similar programs, i.e., Viking Orbiter, Voyager, and Galileo Orbiter, convert them to FY84 $\$$, using the NASA inflation indexes, and subtract the science payload costs, and then divide by $\$ 150 \mathrm{M}$. In order to put the Galileo costs on a comparative basis adjustments were made to account for the inefficiencies due to the several reprogrammings and for the FRG contribution to the RPM. Likewise in the case of Viking and Voyager adjustments were made to account for two flight spacecraft vs one for Galileo.

The intermediate calculations are shown in the attached Table. The $10 \%$ estimate for the incremental cost of a two spacecraft mission may be arguable but is consistent with the estimate we made for building and testing an identical Galileo Orbiter for launch in Jan 86, as well as with earlier estimates for a second spacecraft. The science payload costs were estimated at $10 \%$ for 
Voyager and Viking for convenience, whereas actuals were used for Galileo since they were readily available.

In any event I think a factor of 3 would be conservative and anything less than a factor of $2-1 / 2$ highly unlikely. If I can be of further assistance please call.

Sincerely,

John R. Casani

Manager

Project Galileo

cc: J. C. Beckman

R. J. Parks

July 5,1983

230-JRC: ps-026

Comparative Development Cost Summary

(in millions of dollars)

\begin{tabular}{lccc}
\hline & $\begin{array}{l}\text { Viking } \\
\text { Orbiter }\end{array}$ & $\begin{array}{c}\text { Galileo } \\
\text { Voyager }\end{array}$ & Orbiter \\
\hline Real Year & 235 & 257 & 518 \\
FY 1984 & 652 & 550 & 614 \\
Reprogramming & & & 482 \\
$\begin{array}{l}\text { Retro propulsion } \\
\text { model adjustment }\end{array}$ & & & \\
& & & 533 \\
$\begin{array}{l}\text { Single spacecraft } \\
\text { adjustment }\end{array}$ & $\begin{array}{r}587 \\
(10 \%)\end{array}$ & $\begin{array}{c}495 \\
(10 \%)\end{array}$ \\
$\begin{array}{l}\text { Less science } \\
\text { payload }\end{array}$ & 528 & 446 & 490 \\
Factor & $(10 \%)$ & $(10 \%)$ & $($ actual) \\
\hline & 3.5 & 3.0 & 3.3 \\
\hline
\end{tabular}




\section{APPENDIX C: PRIMITIVE BODY MISSIONS}

\section{Primitive Bodies Study Team}

Laurel L. Wilkening (Chair), University of Arizona Andre Brahic, Observatoire de Paris Don Brownlee, University of Washington Andrea Carusi, Istituto Astrofisica Spaziale, Reparto Planetologia Clark R. Chapman, Planetary Science Institute Michael Festou, Service d'Aeronomie du CNRS Wing-H. Ip, Max-Planck-Institut für Aeronomie H. Loeb, Justus Liebig Universität Daniel Malaise, Universite de Liege David Morrison, University of Hawaii Jack Trombka, NASA Goddard Space Flight Center Donald K. Yeomans, Jet Propulsion Laboratory 


\section{CONTENTS}

SUMMARY OF RECOMMENDATIONS

SCIENTIFIC OBJECTIVES FOR MISSIONS TO THE PRIMITIVE BODIES

COMET RENDEZVOUS WITH ASTEROID FLYBYS

MULTIPLE ASTEROID ORBITER WITH ASTEROID FLYBYS

A TOUR OF MULTIPLE PRIMITIVE BODIES

ADVANCED MISSION: COMET SAMPLE RETURN COMET TARGET SELECTION, by M. Festou and D.K. Yeomans

SOLAR-ELECTRIC PROPULSION, by H. Loeb and E. Stuhlinger

INSTRUMENTATION FOR COMET AND ASTEROID MISSIONS 


\section{SUMMARY OF RECOMMENDATIONS}

\section{Recommended Missions}

The Primitive Bodies Study Group recommends a Comet Rendezvous mission (including asteroid flybys) and a Multiple Asteroid Orbiter with flybys for immediate consideration as international cooperative missions. An attractive alternative to these two missions is a single mission involving a rendezvous with one comet and orbital encounters with two asteroids, the Multiple Primitive Bodies Tour. The Multiple Primitive Bodies Tour and the Multiple Asteroid Orbiter missions require the development of solar-electric propulsion (SEP).

In the area of advanced missions the study group selected the return of a well-preserved and well-characterized sample of a cometary nucleus, the comet sample return, as its top priority. Such a mission also requires SEP.

Although the study group considered some attractive augmentations to the basic missions mentioned above such as the comet atomized sample return and battery-operated penetrators or hard landers for comets or asteroids, they are considered possible additions to the recommended missions, not substitutes for them.

\section{Costs}

The costs for the various missions mentioned above and discussed in detail in the body of the report are given Table C.1. These costs were estimated by J. Niehoff using Science Applications Incorporated cost modeling software. In the modeling process it was assumed that the Comet Rendezvous mission would use a newly designed spacecraft, such as the National Aeronautics and Space Administration (NASA) Mariner Mark II. For missions following the Comet Rendezvous mission, some savings were incorporated in science and spacecraft hardware cost elements because of inheritance from this first mission. The costs of the SEP module 
for the asteroid missions include development and test. However, the comet sample return version is assumed to be a derivative of one of the earlier versions and, hence, includes a cost savings. If there were no SEP precursor mission, the cost of SEP for the comet sample return would increase to $185 \mathrm{M}(1 \mathrm{M}=1$ million U.S. dollars or 1 million European accounting units).

\section{Supporting Recommendations}

Because so many of the recommended missions need the solarelectric propulsion module, the study group strongly urges the development of such a low thrust module as soon as possible.

Because successful international collaboration on the design and execution of a project as sophisticated as a planetary mission requires

collaboration on technical and scientific levels as well as political agreements, the study group recommends (1) continuation of collaborative working relationships between European Space Technology Center (ESTEC) and Jet Propulsion Laboratory (JPL) engineering personnel on specific technical issues relating to solarelectric propulsion, trajectory analysis, thruster-spacecraft integration, etc., and (2) establishment of joint working groups involving European and U.S. scientists and engineers in the area of scientific instrumentation development and mission planning. 


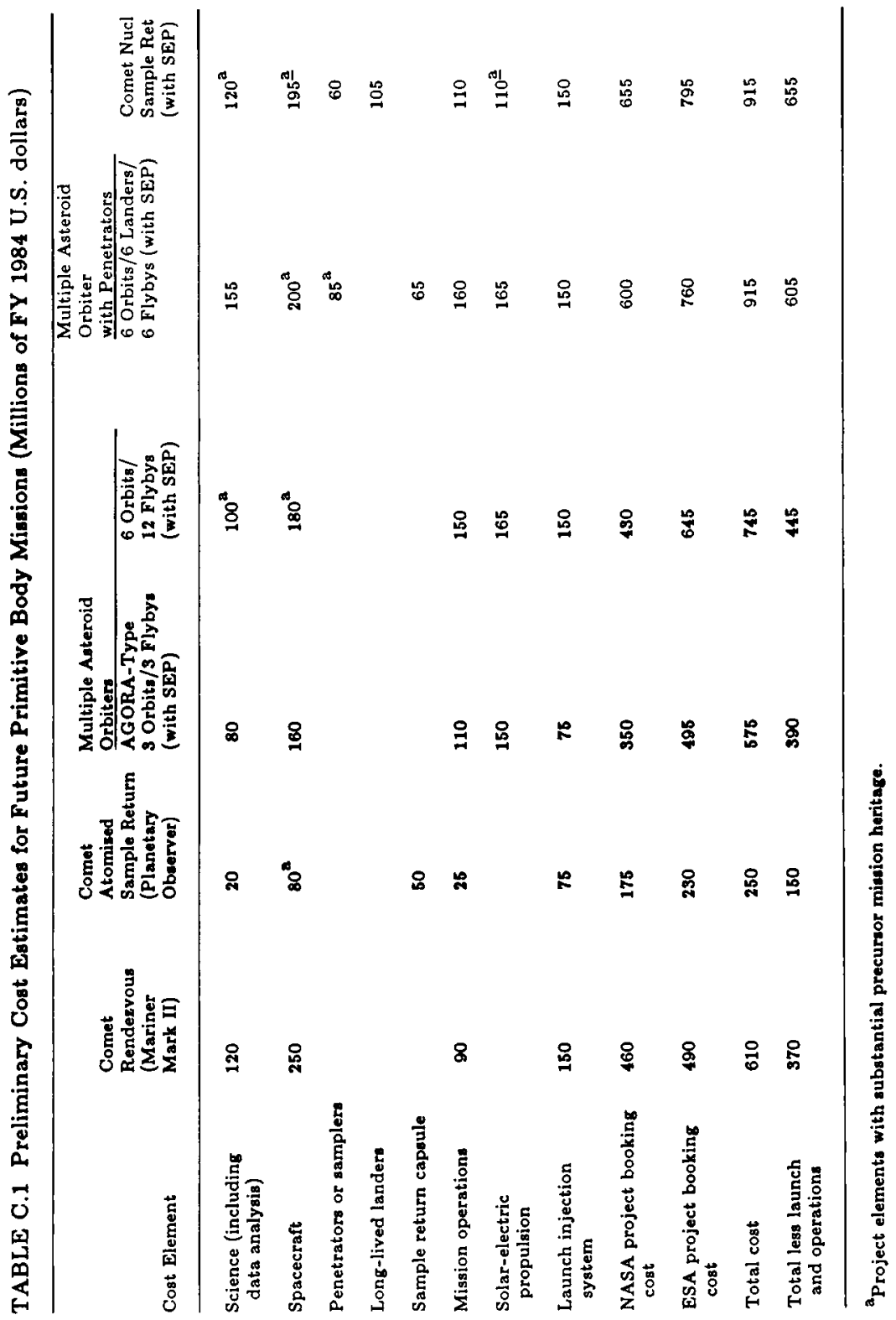




\section{INTRODUCTION}

\section{Importance of Primitive Bodies}

Fundamental to understanding the solar system and its place in the universe is information about how the solar system originated. Present understanding is embodied in various hypotheses concerning the early solar nebula and the collapse and accretion of this mixture of dust and gas to form the planets. The solar nebula hypotheses are based on observations of other stellar systems and the sun, global data on the planets, detailed study of the Earth and moon, and detailed but incomplete data from meteorites that have come to Earth. Some meteorites are thought to preserve solid matter antedating the solar system. They themselves are thought to be relics of some of the processes that resulted in formation of the planets. Meteorites have this distinction because they are pieces of objects, comets, and/or asteroids, which largely escaped the planetary evolution that has obliterated or greatly modified the original material from which planets formed. Yet meteorites have the great disadvantage of being like pages ripped from the books of anonymous authors.

Because the data pertaining to the earliest era of solar system evolution are so fragmentary, and the thirst for knowledge about Earth's beginnings is so great, there is an overwhelming need to visit these solar system objects most likely to provide the missing information, the primitive bodies, the asteroids and comets. The knowledge gained from the study of the primitive bodies will be different from that obtained in the study of the planets and their satellites in that it will be related substantially to questions concerning the origin of the solar system rather than the definition of its present state.

Comets are believed to have formed in the outer portion of the solar system beyond the orbit of Saturn. Temperatures at those distances were probably low enough to have preserved molecules usually associated with planetary atmospheres in the form of ices. These molecules are formed primarily from $\mathrm{H}, \mathrm{C}, \mathrm{O}$, and $\mathrm{N}$, astrophysically important elements for which considerable information 
is available for other regions of the universe. In addition to the icy constituents, comets contain dust and possibly larger rocky fragments. Some fraction of cometary dust may be interstellar dust that predates the formation of solids in our solar system; equally exciting is the possibility that some cometary dust may be some of the first formed solid matter in our solar nebula. In fact, only comets offer the possibility of tracking the whole temperature sequence, highest to lowest, during the early evolution of the nebula.

Comets are also important probes of the interplanetary environment. Their highly ellipitical orbits take them from far beyond Pluto to inside the orbit of Mercury. In the course of this trip, comets respond dramatically to the changing radiation and particle and fields environment by forming transient atmospheres (coma) and tails. Present understanding of these phenomena is very sketchy. Hence, the study of comets presents challenges not only to those interested in origins but also to those interested in the interplanetary environment.

The study of asteroids is considered important to a better understanding of the origin of the solar system because asteroids are small and spectral data suggest that many of them are primitive. Asteroids are also of interest because the main belt of asteroids is located in the transitional zone separating the rocky, terrestrial planets from the gaseous, outer planets. In fact, the spectral properties of typical asteroids vary with heliocentric distance in a way that suggests they preserve an early compositional gradient of the solar nebula. Much of the scientific interest in asteroids pertains to their diversity. In addition to being an apparent remnant population of planetesimals, asteroids, like the meteorites, appear to include not only individuals that have been preserved from early epochs with little modification, but also others whose spectral signatures reflect a surprising degree of geochemical and geophysical evolution, presumably in response to planetary processes operating in the early solar system. Why some asteroids evolved and others did not is of fundamental importance to our understanding of early planetary evolution. Therefore, in targeting asteroids, it is important to explore the several dimensions of diversity by visiting a substantial number of objects of different types.

The diversity of comets and asteroids is well known from direct observations of hundreds of comets and asteroids. Comets 
exhibit a wide range of complex behaviors that are usually interpreted in terms of their history but which may also be due to primordial differences. Asteroids have been classified into eight major spectral groups, reflecting different surface compositions. Many other asteroids do not fit into these classes and represent additional compositions and surface properties. If additional evidence of diversity were needed, then it could be supplied by the meteorites, which must originate in asteroids and/or comets and which range from once molten, slowly cooled nickel nonalloys to fragile water- and organic compound-bearing fragments of soil.

Such a wealth of objects, all of them potential bearers of clues to the processes and nature of the materials preceding and deriving from the early solar nebula and the epoch of planetary accretion, is at once exciting and overwhelming. However, with the scientific knowledge and spacecraft and instrument technology now available, it is quite possible to devise a logical plan for the exploration of the primitive bodies. Although a rather limited set of targets can be selected, there is little chance that too many encounters would lead to redundant results. For example, a rendezvous with a single short-period comet is a necessary next step after the Comet Halley flyby missions; a second rendezvous mission to a different short-period comet with a different history of activity is likely to provide a quite complementary but different set of data because of the different opportunities for measurement and because of diversity among comets.

\section{Appeal of the Primitive Bodies Missions}

In the history of planetary exploration, one group of bodies has thus far escaped yielding its secrets to space probes. Despite the increasing awareness that asteroids and comets are the remnants of the planetary building blocks, and thus contain precious clues about the origin of the solar system, there has been as yet no closeup study of even a single one of these numerous bodies. Spacecraft currently on their way to Comet Halley will begin to rectify this gap in planetary exploration, but the high velocity of that famous comet requires the observations to be made "on the fly."

The study of small bodies in the solar system environment has a long tradition in Europe. This is evident from the prominent roles of European institutions in the experimental and theoretical investigations of meteorites, interplanetary dust, comets, and 
asteroids. Besides ground-based observations and laboratory experiments, attention has now been drawn to space research as exemplified by the European Space Agency (ESA) Giotto mission to Comet Halley and the Agora proposal for an asteroid rendezvous mission under consideration by ESA. Because of planning and execution of these missions, expertise in instrumentation and technology for such planetary projects has been accumulated in Europe. It is, therefore, logical to extend this know-how to followup missions immediately after the Giotto project. We note that a large number of space scientists and astronomers are involved in the Giotto mission. A strong base for cometary research in the aspects of planetology as well as space plasma physics, thus, has been established, and the study group expects that the interest and enthusiasm will be extended to further exploration as discussed within the framework of the present joint study team activities.

Because of the fast flyby nature of the Giotto mission, a number of important questions will not be addressed satisfactorily, for example, the surface structure of the nucleus, the temporal variation of the outgassing process from the cometary nucleus, and the interaction of the cometary atmosphere with the everchanging solar wind. These are issues that can be resolved only by a rendezvous mission. In any event, the first look at the cometary environment by the Giotto mission will allow more appropriate design of the instruments and the mission concept. There are practical reasons to initiate a comet rendezvous mission in the wake of the Comet Halley flybys.

In the United States there is a diverse community of scientists interested in and supportive of the exploration of the primitive bodies. Some of the support is from the astronomers, who have traditionally studied comets and asteroids. Both of these areas of astronomy have showed considerable growth and vigor over the past decade. There is also considerable interest on the part of the scientific community that studies meteorites and interplanetary dust. Finally, anyone interested in the origin and evolution of our solar system has more than a passing interest in the objects most likely to contain the records of those events. 


\section{SCIENTIFIC OBJECTIVES FOR MISSIONS TO THE PRIMITIVE BODIES}

Scientific objectives have been formulated in the course of mission planning for comet and asteroid missions on both sides of the Atlantic. The study group reviewed the objectives formulated previously and proposed syntheses of the objectives, which appear in the sections below for comets and asteroids. These are considered to be overall objectives that would not be satisfied totally in the course of any single mission but that could be addressed by an orderly sequence of well-planned missions.

\section{Scientific Objectives for Comet Missions}

Because of the diversity of comets, the study group stresses that it is important to study the range of cometary phenomena exhibited by various comets.

1. Determine the elemental, isotopic, and mineralogical composition and the physical and geological structure of the cometary nucleus. Characterize the activity and evolution of the nucleus as a function of its heliocentric distance. Determine the bulk density of the nucleus.

2. Better characterize the coma (and the nucleus) through the identification of parent molecules and the chemical and physical processes by which molecules at the surface of the nucleus are transformed in the inner coma. Observe the changes in activity of the coma as a function of time and heliocentric distance.

3. Determine the ratios of ices, low-temperature minerals, and high-temperature minerals among cometary grains; also determine the compositions of individual grains and the spatial distribution of centimeter-sized and larger particles in the central regions of comets. Study the variation in grain composition as a function of distance from the nucleus and the sun, as well as the interaction between plasma and grains.

4. Observe the solar wind interaction with the coma and ion tails. Study the growth and acceleration phenomena in ion tails. 


\section{Scientific Objectives for Asteroid Missions}

Because of the great diversity of asteroids, the study group stresses the importance of studying individual asteroids that represent the range of compositional classes, semimajor axes, sizes, and family membership.

1. Determine the chemical and mineralogical composition of asteroids. Assess the degree of heterogeneity on the surfaces of individual asteroids to investigate evolutionary processes and to study whatever internal portions of asteroids may have been revealed by collisional disruption. Determine the bulk densities of asteroids.

2. Investigate the physical structures of asteroids, including their surface geology and their geophysical configuration with a view to learning about their collisional history and endogenic history.

3. Study the space environment of asteroids, including any accompanying dust, any latent comet-like activity, and evidence concerning the remnant magnetic fields.

4. Investigate the chronology of different stages in the development and evolution of asteroids, both primitive bodies and those that are evolved geochemically. 


\section{COMET RENDEZVOUS WITH ASTEROID FLYBYS}

A comet rendezvous can be carried out using chemical propulsion or low-thrust propulsion systems. This section will describe an example of the least expensive and least technologically challenging approach to comet rendezvous.

\section{Potential Targets for a Cometary Rendezvous Mission}

In recent years the JPL team, led by D. Yeomans, has issued a few lists of comet mission targets. In these lists, various well-observed short periodic comets were ranked by their maximum brightness near perihelion (at a standard distance from the Earth of 1 astronomical unit (AU)). Only those comets with wellunderstood orbital motions were considered. The list of possible target comets is presented in Table C.8. From this list the study group selected those comets that (1) are bright at or near perihelion, (2) have perihelion passages between 1995 and 2000, (3) have good ground-based viewing conditions near the perihelion passages of interest, and (4) are in orbits that allow spacecraft rendezvous without excessive amounts of fuel expenditures (are in low inclination and eccentricity orbits). Using these criteria, the study group eliminated all opportunities except the following:

$\begin{array}{ll}\text { P/Encke } & 1997 \\ \text { P/Kopff } & 1996 \\ \text { P/Honda-Mrkos-Pajdusakova } & 1995 \\ \text { P/d'Arrest } & 1995 \\ \text { P/Tempel 2 } & 1999 \\ \text { P/Wild 2 } & 1997 \\ \text { P/Churyumov-Gerasimenko } & 1996 \\ \text { P/Tuttle-Giacobini-Kresak } & 1995\end{array}$

Nonperiodic comets or newly discovered comets are not considered here because their returns are not predictable sufficiently far in advance to allow the necessary preparation. It is highly desirable that the target comet be observable from ground-based observatories during the rendezvous period. Ground-based data on the large-scale phenomena will complement the near-nucleus 
spacecraft data, and the in situ data will help calibrate the groundbased observations.

This set of targets is composed of very diverse objects, and none of the eight comets displays the full range of comet phenomena (coma, well-developed dust, and ion tails). Differences among them are either real, more or less gas in the coma, more or less dust near the nucleus, or induced by the path followed by the comet around the sun. As an example, some may exhibit an ion tail because they have a short perihelion distance (the ionizing solar flux is large), while others display the same ion tail at larger heliocentric distances because of a higher gas production rate that compensates for the lack of UV photons (P/Encke and $\mathrm{P} /$ Honda-Mrkos-Padjusakova versus $\mathrm{P} /$ Churyumov-Gerasimenko and $\mathrm{P} / \mathrm{Kopff}$ ). Further discussion of the natures of the target comets and additional criteria for selection can be found in the section on comet target selection below.

\section{A Comet Rendezvous Example: P/Tempel 2}

In addition to the criteria discussed above and in the section on comet target selection, the following constraints were also considered desirable:

1. Good opportunities should exist for ground-based observations prior to launch.

2. The spacecraft should not require extensive modifications for thermal control, nor should the mission strategy be dominated by thermal constraints.

3. The spacecraft should have an early launch, as short a flight time as possible, and as large a payload mass margin as possible.

4. Good opportunities should exist for using the rendezvous spacecraft as a beacon for an atomized sample return spacecraft. While this concept is not treated explicitly in this section, it would be a mistake to choose a target comet that precludes an atomized sample return possibility.

5. The science return of any comet rendezvous mission would be greatly enhanced if one or more close flybys of main-belt asteroids could be identified en route to the comet.

From the eight comets selected in the previous sections, attempts were made to find optimum trajectories to rendezvous with 
active comets at least 50 days prior to perihelion. Using the criteria employed in the previous section and those mentioned above, the number of attractive rendezvous possibilities was considerably reduced (C.W. Yen, unpublished presentation, 1983). Three opportunities were considered for further study:

1. A 1999 rendezvous with $P /$ Tempel 2 (1994 launch),

2. A 2000 rendezvous with $P /$ Encke (1996 launch), and

3. A 2001 rendezvous with $\mathrm{P} /$ Honda-Mrkos-Pajdusakova (1995 launch).

Before discussing these three opportunities, a 1996 rendezvous opportunity with $\mathrm{P} / \mathrm{Kopff}$ deserves special consideration. If the post-1991 launch constraint can be relaxed, then this mission, with its July 1991 launch, would be attractive. At this writing the magnitude estimates of this comet are considerably brighter than one would have expected from its previous apparitions. As the comet approaches and recedes from perihelion (August 1983), the comet's magnitude will be monitored closely. If the comet remains systematically brighter during this entire near-perihelion apparition, then it would appear that this comet's ability to produce gas has significantly increased, and $\mathrm{P} / \mathrm{Kopff}$ would become one of the most attractive rendezvous opportunities in the 1995-2000 period. However, for the present we will restrict ourselves to the the $\mathrm{P} /$ Tempel 2, Encke, and Honda-Mrkos-Pajdusakova opportunities mentioned above.

While $\mathrm{P} /$ Encke is the most active comet near perihelion, this mission requires a Jupiter gravity assist for which a limited number of asteroid flyby opportunities are available. In addition, the ground-based viewing of the comet is very poor in 2000 , and the spacecraft will require modifications for thermal control, and an atomized sample return mission to $P$ /Encke would be very difficult. Comet Honda-Mrkos-Pajdusakova, though somewhat more active than $\mathrm{P} /$ Tempel 2 at perihelion, suffers from poor groundbased observing conditions in 2001 and has 30 percent less payload mass margin than the 1999 Tempel 2 opportunity. The opportunity for a rendezvous mission with P/Tempel 2 in 1999 provides an excellent illustration of how such missions would be conducted.

The 1999 rendezvous opportunity with $\mathrm{P} /$ Tempel 2 has excellent asteroid flyby opportunities and the largest spacecraft mass margin of the three missions. This comet has been well observed 
TABLE C.2 Comet Tempel 2 Rendezvous and Asteroid Flybys

\begin{tabular}{ll}
\hline Event & Date \\
\hline Launch & July 28, 1994 \\
Flyby of 42 Isis (arbitrarily close $-9.4 \mathrm{~km} / \mathrm{s})$ & March 4, 1995 \\
Flyby of 1247 Memoria (8.1 km/s) & August 31, 1995 \\
Rendezvous with P/Tempel 2 perihelion (50 days) & July 21, 1999 \\
\hline
\end{tabular}

for over a century, and its orbit is well determined. The groundbased viewing conditions for P/Tempel 2 in 1999 are excellent, with the comet appearing in a dark sky and within $1 \mathrm{AU}$ of the Earth for 5 months near perihelion. There are also good groundbased viewing opportunities in 1983-1984 and 1988, well ahead of launch. This mission has the earliest launch, and opportunities for atomized sample returns have been identified for this comet at perihelion. In addition, this comet's spectra indicate a strong continuum, implying a good deal of dust available for capture during the collection period of an atomized sample return spacecraft.

Table C.2 presents the proposed mission time line of a rendezvous with $\mathrm{P} /$ Tempel 2 with en route close flybys of main-belt asteroids 42 Isis and 1247 Memoria. Table C.3 briefly summarizes the physical and orbital data on these three objects.

\section{Rendezvous Mission Scenario}

A possible rendezvous mission scenario might include four phases.

1. Rendezvous and Quick-Look Approach, Perihelion-50 Days to Perihelion-10 Days ( $P-50$ to $P-10)$. At rendezvous the comet has a heliocentric distance $(r)$ of $1.56 \mathrm{AU}$ and a geocentric distance $(\Delta)$ of $0.66 \mathrm{AU}$. If the dust environment permits, the spacecraft should make an initial sunside flyby to within $100 \mathrm{~km}$ and then back away to a safe $4,000-\mathrm{km}$ distance while monitoring the rapidly increasing cometary activity. A rough mass estimate is made during this phase, and the hostility of the near-nucleus region is assessed.

2. Perihelion Monitoring (P-10 to $P+90)$. After passing perihelion at $r=1.48 \mathrm{AU}$, the comet's peak activity occurs near 
TABLE C.3 Characteristics of Comet Tempel 2 and Asteroids 42 Isis and 1247 Memoria

\begin{tabular}{|c|c|c|c|}
\hline & P/Temple 2 & 42 Isis & 1247 Memoria \\
\hline Est. radius (km) & 1.5 & 42 & \\
\hline Spectra & $\begin{array}{l}\text { strong continuum } \\
\mathrm{CN}, \mathrm{C}_{2}, \mathrm{C}_{3}, \mathrm{CO}^{+}\end{array}$ & S type & C type \\
\hline First observed & $1873^{2} 3$ & 1856 & 1932 \\
\hline Orbital period (years) & 5.3 & 3.8 & 5.6 \\
\hline Perihelion distance ( $\mathrm{AU}$ ) & 1.48 & 1.89 & 2.61 \\
\hline Aphelion distance ( $\mathrm{AU})$ & 4.73 & 2.99 & 3.66 \\
\hline $\begin{array}{l}\text { Absolute magnitude } \\
\text { at perihelion }\end{array}$ & 10.0 & 10.2 & 14.8 \\
\hline Photometric behavior & \multirow{2}{*}{\multicolumn{3}{|c|}{$\begin{array}{l}\text { Rapid brightness increase } \\
-80 \text {-day preperihelion } \\
\text { Sunward fan-shaped coma } \\
\text { Maximum visual coma diameter } \\
-10^{5} \mathrm{~km} \\
\sim 10^{28} \text { (maximum) } \\
\text { (molecules/s) }\end{array}$}} \\
\hline Est. production rate & & & \\
\hline
\end{tabular}

$P+20$. From the sunward hemisphere, the spacecraft observes the comet at various phase angles from a safe distance.

3. Post-Perihelion Monitoring $(P+90$ to $P+150)$. As the cometary activity subsides, several sunside passes of the spacecraft past the nucleus are made to count and analyze dust particles. The dust instruments will operate when instruments susceptible to dust contamination (neutral mass spectrometer (NMS), infrared mapping spectrometer (IRS), and the camera) are shielded. If the environment allows, the sunside passes will be made at successively closer ranges. The atmospheric instrumentation will also be most active during this phase.

4. Nucleus Orbiter $(P+150$ to $P+300)$. As the comet returns to its quiescent state $(r \sim 1.9)$, the investigative emphasis will switch to nucleus-intensive studies. While the spacecraft orbits the comet's nucleus at lower and lower altitudes, the camera, IRS, $\mathrm{x}$-ray and gamma-ray spectrometers, IR radiometer, and altimeter will be operating nearly continuously. Chemical and mineralogical composition measurements will be made, the surface will be mapped, the mass determined, and the bulk density computed. 
Anticipated Scientific Results

A rendezvous mission to $P /$ Tempel 2 would address substantial portions of comet science objectives 1-3 (see section above on scientific objectives for comet missions) for one particular comet and form a basis of in situ measurements to allow more meaningful interpretation of subsequent ground-based data on different comets. A rendezvous mission is the only way of studying the source of the cometary phenomena, the nucleus, as it evolves with heliocentric distance. Although much will be learned from the 1985-1986 spacecraft encounters with $\mathrm{P} /$ Halley and $\mathrm{P} /$ GiacobiniZinner, the characterization of the nucleus' activity with orbital position will have to await the results of long-term in situ observations. The fundamental elemental, isotopic and mineralogical composition and structure of the cometary nucleus can only be addressed in rendezvous and sample return observations. The longer dwell times of a rendezvous mission will allow much improved identifications of parent molecules and provide compositions and spatial distributions for the coma dust particles. Through in situ magnetometer and plasma instrument measurements and corollated ground-based observations, much of the fourth science objective will be addressed as well.

The mission scenario includes arbitrarily close flybys of two main-belt asteroids of different spectral classes and sizes. Important asteroid science objectives would be addressed by this mission. Mineralogical composition could be measured and mapped along with the bulk density. The size, shape, spin period, cratering history, surface morphology, dust environment, and signs of latent activity would be investigated for two diverse asteroids. Thus, the majority of the science objectives for the comet and a significant portion of asteroid mission objectives could be accomplished with one mission in the lowest cost category. By sharing the costs between Europe and the United States, the fiscal burden would be further tightened for both groups.

One possible division of responsibilities would be that the Europeans build the spacecraft propulsion and attitude control system while the United States builds the remaining spacecraft including the on-board computers and data system. The science payload would be shared equally. 


\section{Atomized Sample Return from a Comet Flyby}

An important augmentation that could be made to a comet rendezvous is the atomized sample return, which could employ a rather simple spacecraft that would home on the rendezvous spacecraft, fly by the comet, sampling dust in its vicinity, and return to Earth.

At the present time there are several meteoroid capture cell collectors being built for use on recoverable Earth-orbiting spacecraft. During exposure, a meteoroid enters a capture cell by penetration of a thin front diaphragm, the meteoroid is vaporized, and the resulting vapor condenses on the interior walls of the cell. Cell dimensions can be chosen so that impacting particles are compartmentalized into individual cells. Condensed matter in the cells is then returned to the laboratory and analyzed by techniques such as isotope dilution mass spectroscopy, the ion microprobe, or the synchrotron $x$-ray fluorescence. The flexibility and sophistication of available laboratory techniques allow the determination of the elemental and isotopic composition of individual particles to be made at high precision with documentable reliability. Capture cells have been designed to collect particles ranging in size from a micrometer to a millimeter.

Other collection options are also possible for a flyby mission. For example, if encounter velocity is relatively low, abundant particle residue will be retained in crater linings in certain substrates such as gold. It is also possible that some particles may be decelerated nondestructively by low-density media.

Application of capture cell technology to a comet requires flying a capture cell array to a comet for a fast flythrough of the coma. The spacecraft can be launched on a free return trajectory and recovered following either direct atmospheric entry in a probe or by aerobraking and recovery with the Shuttle. The collector would cover the entire leading edge of the spacecraft during the flyby and would also serve as the meteoroid impact shield. In addition to other advantages this allows the collector to capture the largest particle that impacts the spacecraft. This permits close flyby and collection of comparatively large particles that could not possibly be analyzed by in situ instruments. Large particles are important because their compositions are probably good indicators of the bulk nucleus composition. 
The atomized sample return from a cometary coma is the simplest possible sample return from any solar system body because it does not require landing and surface operations. The collection technique is destructive, but the analysis gives the elemental and isotopic composition of individual particles. For particles that are composed of a single mineral, the composition can be used to infer the mineral.

Various laboratory studies are under way to optimize the design of capture cells. Simulations have been done on test cells using light gas guns, electrostatic accelerators, and pulsed electron beam facilities. The basic collection concept has been validated by these tests. Future refinements will incorporate data from capture cells exposed in Earth orbit. The first meteoroid impacts registered on a recovered capture cell experiment were found on a U.K. experiment flown on the third Shuttle flight.

The instrumentation complement recommended by the study group is shown in Table C.4 and described in the section entitled "Instrumentation for Comet and Asteroid Missions." 
TABLE C.4 Scientific Instruments for Comet Rendezvous Missions

\section{Essential}

Imaging charge-coupled device

Infrared mapping spectrometer

Neutral mass spectrometer

Dust counter

Dust chemical analyzer

$\mathrm{X}$-ray and gamma-ray spectrometer

Ion mass spectrometer

IR radiometer

\section{Desirable}

UV spectrometer

IR spectrometer

Visible spectrometer

Magnetometer

Plasma analyzer

Plasma wave analyzer

Radar sounder

Radio science

Test Mass Experiment (asteroid flyby only)

Altimeter (essential for navigation)

\section{Atomized Sample Return}

Collector

(no additional instruments are required) 


\section{MULTIPLE ASTEROID ORBITER WITH ASTEROID FLYBYS}

Asteroids are among the most easily reached objects in the solar system, contain important clues to the origin and early evolution of the solar system, are potential sources of valuable raw materials in easily accessible form, and are of interest for their historical role of colliding with the Earth and other terrestrial planets. Due to their scientific interest, a first exploration mission of the asteroids is considered by the study group as a very high priority.

\section{Asteroid Target Selection Criteria}

It is essential in the exploration of asteroids to examine individuals that span the range of diversity in composition, size, spin, family membership, and heliocentric distance. The proposed Multiple Asteroid Orbiter mission permits the possibility of visiting several different asteroids by flybys while accomplishing intensive study by orbiting two to four asteroids. Given the inexhaustible supply of possible multiple asteroid tours that change with launch year, it is not possible to identify a best tour. Nevertheless, the study group can specify the appropriate criteria and present an example.

Of prime importance are the major compositional types. A tour should visit at least one of each type that represents a few percent or more of the asteroid population. The predominant $\mathrm{C}$ and $S$ types must be sampled, and the $M, P$, and $D$ types have high priority (although $P$ and $D$ are not located in the inner belt). In addition, several of the unusual asteroid types that probably represent a high degree of differentiation should be studied (e.g., 4 Vesta, 44 Nysa, perhaps 349 Dembowska). Also of great interest is the variation in size. Among all the objects to be visited, it is important to ensure that both large and small members of the $\mathrm{C}$ or C-like classes are included, to determine the dependence of size in modifying the presumably primitive nature of these objects. It would be extremely important to study the interiors of any asteroids revealed by catastrophic collisions. $M$-type (and perhaps 
some S-type) asteroids may be revealed cores, although this is not certain. Certainly the list of potential targets should include any asteroids whose light curves, varying composition with rotational chase, and other characteristics suggest they are likely disrupted bodies.

The multiple asteroid flybys that are a part of all of the proposed primitive bodies missions will contribute greatly to investigating the diversity of the asteroidal population. With prospects for several flybys, it should be possible to visit many of the different groups discussed above. The task of selecting the much smaller number of asteroids that will be intensively studied by orbiter spacecraft is especially important. Based on current understanding, the study group places highest priority on orbiter missions to one primitive (C-type or C-like) asteroid and one highly evolved asteroid (4 Vesta is the outstanding example). In a list of four targets for orbiters, it is also essential to include one S-type (the predominant type in the inner belt, possibly related to the ordinary chondrite meteorites) in addition to the C-type and the differentiated body, with the fourth target selected to explore some other dimension of asteroidal diversity. Some emphasis for both flyby and orbiter targets should be given to larger bodies, for they will afford better subjects for some studies and may reveal a wider diversity of structures bearing on asteroidal processes than would a tiny fragment.

\section{Propulsion Options}

It should be emphasized that the greater the propulsion capability, the larger the number of asteroids that can be visited in any period of time. Furthermore, the nature of the propulsion system strongly influences the number of available targets and the mission flexibility. There are two possibilities: the use of conventional chemical propulsion and the use of solar-electric propulsion.

Using chemical propulsion, the corrections of trajectories are instantaneous and easy to perform. The total change of velocity is limited by the maximum mass of fuel that can be launched from the Earth. Depending on the launcher, Ariane or the Shuttle-Centaur (G), the total change of velocity is of the order of 2 to 4 kilometers per second $(\mathrm{km} / \mathrm{s})$ for a spacecraft of 500 to 1,000 kilograms $(\mathrm{kg})$ with a scientific payload of the order of 100 to $300 \mathrm{~kg}$. The maximum velocity increment that can be obtained with chemical 
propulsion allows only one or two rendezvous with an asteroid approaching or crossing the orbit of the Earth (called Apollo-Amor asteroids). The opportunities for flybys of other asteroids are relatively low because the spacecraft path is such that it will cross the asteroid belt quickly. Although the specific scientific interest of Apollo-Amor asteroids is high (possible extinct cometary nuclei, intermediate parent bodies of meteorites, potential utilization), a mission to the Apollo-Amor asteroids cannot satisfy the scientific objectives of sampling a diversity of primitive objects presumably still located in orbits near where they formed.

Use of solar-electric propulsion (SEP) increases the number of orbiter opprtunities and the flexibility in the choice of targets. SEP systems have been under development in the United States and in Europe for more than two decades. Energy is provided by a solar array, with or without a solar concentrator. Mercury vapor or an inert gas is ionized either by electron impact, or by an inductively coupled, electrodeless discharge within a vessel, and ions are accelerated and expelled by an electric field. The SEP is a high-energy, low-thrust device, which, working continuously, provides a large velocity increment on the order of 15 to $20 \mathrm{~km} / \mathrm{s}$ after 1 to 2 years of operation. Such a propulsion system cannot be used farther than 2.5 to $3 \mathrm{AU}$ from the sun, but within its operating limits, it provides almost infinite flexibility for multiple target orbital missions to asteroids. A more thorough description of SEP development is provided in the section below on solarelectric propulsion.

Once a SEP spacecraft is in the main belt, it is possible to have flybys in between two orbital encounters. The estimated rate of encounters (including orbital encounters) is about one per year. This means it is possible to have a good detailed analysis during orbit and, in addition, a quite valuable sampling of asteroid sizes, types, and families. Without question the most effective exploration approach to asteroid studies would employ SEP technology.

\section{An Example of a Multiple Asteroid Orbiter with Asteroid Flybys}

The number of possible mission sequences using SEP that fit the target selection criteria is enormous. Hence, Table C.5 presents one example that is similar to the Agora mission presently being studied by the European Space Agency (ESA) and that represents 
TABLE C.5 Multiple Asteroid Rendezvous with Asteroid Flybys Example

\begin{tabular}{|c|c|c|}
\hline Time Line & $\begin{array}{l}\text { Body } \\
\text { (Type, Radius) }\end{array}$ & Encounter Type \\
\hline Feb. 13, 1993 & Earth & Launch \\
\hline Oct. 6,1994 & $\begin{array}{l}113 \text { Amalthea } \\
(\mathrm{SX}, 24 \mathrm{~km})\end{array}$ & Flyby $\left(v^{a}=3 \mathrm{~km} / \mathrm{s}\right)$ \\
\hline $\begin{array}{l}\text { June } 13,1995 \\
\text { Sept. 21, } 1995\end{array}$ & $\begin{array}{l}4 \text { Vesta } \\
(V, 288 \mathrm{~km})\end{array}$ & Orbiter \\
\hline March 19, 1996 & $\begin{array}{l}313 \text { Chaldaea } \\
(C, 61 \mathrm{~km})\end{array}$ & Flyby $(\mathrm{v}=6 \mathrm{~km} / \mathrm{s})$ \\
\hline $\begin{array}{l}\text { Oct. } 25,1996 \\
\text { Feb. } 2,1997\end{array}$ & $\begin{array}{l}17 \text { Thetis } \\
(\mathrm{S}, 49 \mathrm{~km})\end{array}$ & Orbiter \\
\hline June 2,1997 & $\begin{array}{l}415 \text { Palatia } \\
(C, 46 \mathrm{~km})\end{array}$ & Flyby $(v=7 \mathrm{~km} / \mathrm{s})$ \\
\hline $\begin{array}{l}\text { Sept. 1, } 1998 \\
\text { Jan. 12, } 1999\end{array}$ & $\begin{array}{l}449 \text { Hamburga } \\
(C, 38 \mathrm{~km})\end{array}$ & Orbiter \\
\hline
\end{tabular}

${ }^{a}$ Flyby velocity.

modest propulsion requirements. It employs an Ariane launch and an SEP system with a 20 kilowatt (kw) array and 5 thrusters. The entire mission, from launch to completion, requires only 6 years. The three asteroids orbited satisfy minimal requirements in terms of the diversity of types and sizes. Asteroid 4 Vesta is a geochemically evolved asteroid that is hypothesized to be the source of certain achondritic meteorites. Presumably, it was partially melted by a strong source of heat very early in solar system history. At $556 \mathrm{~km}$ in diameter, it is the second largest asteroid and the only one that can been seen by the naked eye from Earth. The mission provides opportunities for study of three C-types and two S-types. Asteroid 17 Thetis is a typical S-type. 
Mission Scenario

The gravity of asteroids larger than $10 \mathrm{~km}$ in diameter is large enough that the station-keeping strategies typically assumed for comet rendezvous missions are less appropriate than orbiting. The orbital characteristics of missions to asteroids of various sizes is given in Figure C.1. Given these characteristics, the following mission scenario is one that could be anticipated.

1. Slowly approach the asteroid from $50,000 \mathrm{~km}$. During this phase ( $\sim 3$ days), low-resolution imaging will determine object size, shape, rotation rate, and polar axis. Mass spectrometers, plasma instruments, and dust sensors will monitor the asteroidal environment.

2. Establish a polar observation orbit with a period at least several times longer than the asteroid rotation period for global medium resolution coverage. X-ray spectroscopy and the IR mapper will begin to provide compositional data on the surface and select areas for intensive study.

3. When global coverage is complete, transfer to a low-altitude circular orbit that is nearly resonant with the rotating object, so sites of specific interest can be studied at highest resolution with imaging and mapping instruments. In this phase the gamma-ray spectrometer can acquire bulk composition data.

This scenario does not explicitly include flybys, at least one of which would be expected to occur between each orbital encounter. In the flyby mode with a relative speed at closest approach of 3 to $10 \mathrm{~km} / \mathrm{s}$ and with a minimum distance of less than $1,000 \mathrm{~km}$ above the surface, it would be possible to obtain valuable information about the size, shape, spin period, mass, density, cratering history, surface morphology, mineralogy, and magnetic field.

The orbital mode provides specific advantages because the gamma-ray spectrometer can operate. Global coverage of the asteroid will be possible with all instruments with very good spatial resolution. The mass, volume, and density of the asteroid can be determined with more precision during an orbital encounter, and higher order harmonic terms of the gravity field may also be determined. 


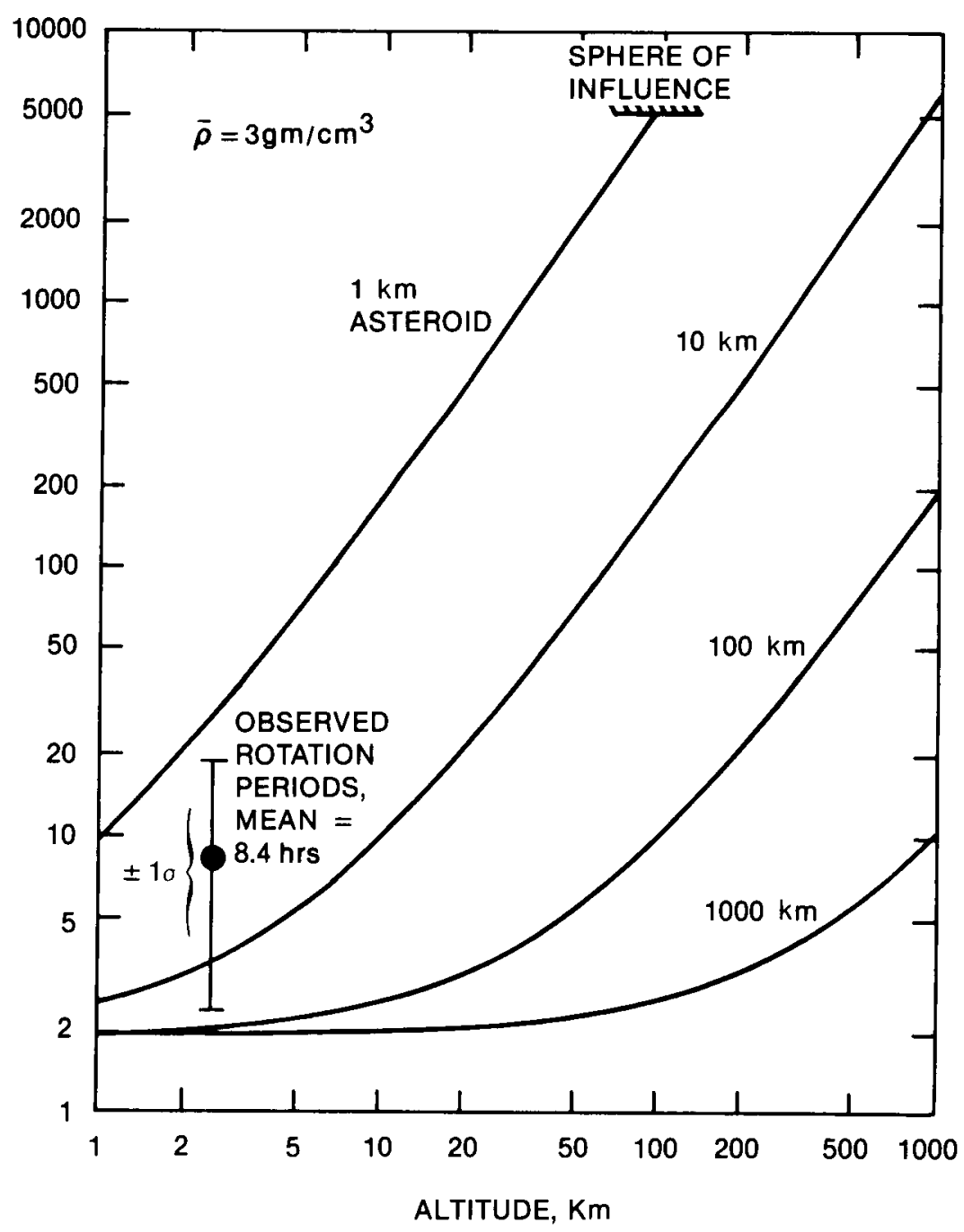

FIGURE C.1 Orbits around asteroids (courtesy John Niehoff, SAI, 1978).

Anticipated Scientific Results

Even the minimal mission that has been described above will permit the examination of about six asteroids, three of them in the orbiter mode. With a more advanced propulsion system and using a Shuttle-Centaur (G') launch, four to six asteroids could be orbited and four to six flybys could be added. This would 
provide a correspondingly more comprehensive approach to the requirements of studying the diversity of asteroids. Even in the minimal mission the asteroids orbited satisfy the requirements to study diversity in composition of the major types. The additional flyby encounters would probably allow the observation of another type and address some of the requirements to study diversity in other characteristics such as size and shape.

Remote sensing instruments, gamma-ray, x-ray, and IR spectrometers, will address the first objective of helping to determine the chemical and mineralogical compositions of the asteroid surfaces. Together with imaging in the orbiting mode, the heterogeneity of the surface and surface morphology, including evidence of igneous processes, presence of regoliths, and other effects of cratering will be investigated. Volume determinations from imaging and mass determination from trajectory analysis will permit the bulk density to be determined.

In the flyby mode the masses can be determined by the test mass experiment and the volume by imaging, allowing a determination of density. Optical imaging and infrared spectrometry do not require a long acquisition time. New advances in $x$-ray spectroscopy detectors reduce the acquisition time to the point that encounters with the parameters cited above could provide information on the $\mathrm{Mg}, \mathrm{Si}$, and possibly $\mathrm{Na}, \mathrm{C}$, and $\mathrm{O}$ relative compositions.

During the approach phase of flybys and orbital encounters, and orbiting phase of orbital encounters, the dust counters and plasma instruments will monitor the asteroidal environment for evidence of outgassing or presence of ejecta debris.

The instrument payload for the Multiple Asteroid Orbiter mission is given in Table C.6. It should be noted that this is a subset of the instruments proposed for the Comet Rendezvous mission.

\section{Asteroid Rendezvous with Penetrators}

An attractive major augmentation to asteroid orbiter missions would be the addition of penetrators. Suitably instrumented penetrators will provide compositional data on asteroids that will allow the identification of meteorite types with specific asteroids. This would be indirect by a sample return mission. Studies carried out on the use of penetrators for planetary missions have indicated 
TABLE C.6 Scientific Instruments for Asteroid Orbiter and Flyby

\section{Essential}

Imaging charge-coupled device

$\mathrm{X}$-ray and gamma-ray spectrometer

Infrared mapping spectrometer

Magnetometer

Test mass experiment (flyby only)

Desirable

Dust counter

Infrared radiometer

Radar sounder

Ion mass spectrometer

Plasma analyzer

Electron detector

Altimeter (essential for navigation)

Scientific Instruments for Asteroid Penetrators

(battery operated)

Accelerometer

Elemental composition analyzer

(pulsed neutron or V-backscatter)

Imaging

Metal detector

that stratigraphy, chemical abundance, and imaging may be the most significant scientific investigations that can be carried out during an asteroid rendezvous mission. Since temporal variations in the scientific objectives just enumerated are not expected, the penetrator operation time can be quite limited.

The basic penetrator design is rocket shaped with a bullet-like nose and a flared conical aft section. When it penetrates the surface, a small afterbody separates and remains at the surface, where scientific observations can be made and data transmission can be accomplished. The forebody and afterbody are connected by an umbilicus that is deployed from a storage area in the forebody. Since shorter operation times are being considered, the penetrator can be powered using batteries stowed in the forebody.

Penetrators have never been studied in detail for asteroid missions. Most of the study of the use of penetrators for planetary missions has been in the context of Mars, and it is clear that some modifications of the martian mission scenarios and the 
planned hardware would be necessary for asteroids. In particular, the acceleration and braking environments, i.e., gravity field and atmosphere, are completely different. The study group concluded that the problems with targeting and communication make the use of penetrators for flyby targets impractical.

A number of scientific instruments have been studied, and in some cases, component tests have already started for use in a penetrator configuration. The instrument complement recommended by the study group is listed in Table C.6 and described in the section below on instrumentation for comet and asteroid missions. 


\section{A TOUR OF MULTIPLE PRIMITIVE BODIES}

The Multiple Primitive Body Tour is especially appealing because it combines the essential elements of missions previously described for asteroids and comets into a single mission. That mission could consist of rendezvous encounters with an Earthapproaching asteroid, an asteroid in the main belt (or two mainbelt asteroids), and a periodic comet, plus flyby encounters with a couple of other main-belt asteroids. It is cost effective because it requires only a single spacecraft and a single launch. However, the data returned would necessarily be less in quantity than those obtained by separate asteroid-intensive and comet-intensive missions because fewer objects could be encountered.

The Multiple Primitive Body Tour would begin the serious study of comets and asteroids by employing rendezvous techniques. By combining comets and asteroids within a single mission, scientists can take advantage of the similar instruments needed to study the central parts of each (the comet nucleus and the asteroid) while also permitting the option to study the space environment of an asteroid the same way a comet's coma will be probed. (It is possible that some asteroids continue to de-gas, like very inactive comets.)

The mission takes advantage of a single launch from Earth and utilizes the ion propulsion system to reach rendezvous with several of these diverse bodies. It is expected that serious study of one comet nucleus and two or more asteroids can be accomplished before the turn of the century. It is difficult to find a currently known Earth-approaching asteroid to include in the tour until after the comet rendezvous late in the mission, but circumstances may prove more favorable in a few years, once several approaches to discovering many more Earth approachers have been carried out.

There are several potential comets for study, although the options are fairly limited. $\mathrm{P} /$ Tempel 2 is a likely target for the late $1990 \mathrm{~s}$. Asteroid targets are readily available for rendezvous prior to the cometary encounter. It is thought best to examine 
one of the presumed primitive C-type asteroids as well as one of the evolved bodies.

The scientific objectives of the Multiple Primitive Body Tour are to explore and compare a diversity of small bodies (including a main-belt asteroid or two, a comet, and perhaps an Earthapproaching object) in order to determine their physical and chemical nature and to study preserved evidence concerning early solar system processes. The rendezvous-mode investigations will be relied on for the chief encounters, but the mission may be richly supplemented by accomplishing one or more flybys of asteroids during other phases of the mission.

The science objectives for this mission are addressed by the same experiments required to study each type of body separately, as detailed in the study group's other mission descriptions. Here, with the Multiple Primitive Body Tour, the focus can be on intellectual cross fertilization between scientists interested in comets, on the one hand, and asteroids, on the other. Certainly, in some ways comets and asteroids are variations on a theme.

One scenario that has been studied is given in Table C.7. The mission involves Shuttle-Centaur (G') launch in mid-1990 and employs SEP. During the 10-year span of the mission, seven asteroids and $P /$ Tempel 2 are encountered. 
TABLE C.7 Example of a Multiple Primitive Bodies Tour

\begin{tabular}{|c|c|c|}
\hline Time Line & $\begin{array}{l}\text { Body } \\
\text { Type, Radius }\end{array}$ & Encounter Type \\
\hline June 27,1990 & Earth & Launch \\
\hline March 26, 1991 & $\begin{array}{l}296 \text { Phaetusa } \\
(\mathrm{S}, 5 \mathrm{~km})\end{array}$ & $\begin{array}{l}\text { Flyby } \\
\left(v^{g}=4 \mathrm{~km} / \mathrm{s}\right)\end{array}$ \\
\hline Nov. 1, 1991 & $\begin{array}{l}1127 \mathrm{Mimi} \\
(\mathrm{C}, 40 \mathrm{~km})\end{array}$ & $\begin{array}{l}\text { Flyby } \\
(\mathrm{r}=6 \mathrm{~km} / \mathrm{s})\end{array}$ \\
\hline $\begin{array}{l}\text { July } 22,1992 \text { and } \\
\text { Nov. } 1,1992\end{array}$ & $\begin{array}{l}4 \text { Vesta } \\
(\mathrm{V}, 288 \mathrm{~km})\end{array}$ & Orbiter \\
\hline Aug. 12,1993 & $\begin{array}{l}60 \text { Drakonia } \\
\text { (CMEU, } 10 \mathrm{~km} \text { ) }\end{array}$ & $\begin{array}{l}\text { Flyby } \\
(v=5 \mathrm{~km} / \mathrm{s})\end{array}$ \\
\hline $\begin{array}{l}\text { March 11, } 1996 \text { and } \\
\text { June 10,1996 }\end{array}$ & $\begin{array}{l}1131 \text { Porzia } \\
\text { (Amor asteroid, } \\
3 \mathrm{~km})\end{array}$ & Orbiter \\
\hline June 12,1997 & $\begin{array}{l}625 \text { Xenia } \\
(15 \mathrm{~km})\end{array}$ & $\begin{array}{l}\text { Flyby } \\
(\mathrm{v}=4 \mathrm{~km} / \mathrm{s})\end{array}$ \\
\hline July 27, 1998 & $\begin{array}{l}529 \text { Preziosa } \\
(\mathrm{SU}, 11 \mathrm{~km})\end{array}$ & $\begin{array}{l}\text { Flyby } \\
(\mathrm{v}=6 \mathrm{~km} / \mathrm{s})\end{array}$ \\
\hline $\begin{array}{l}\text { July } 19,1999 \text { and } \\
\text { Jan. } 19,2000\end{array}$ & Comet Tempel 2 & Orbiter \\
\hline
\end{tabular}

${ }^{a}$ Flyby velocity. 


\section{ADVANCED MISSION: COMET SAMPLE RETURN}

The study group considered the augmentations of the atomized sample return from a comet and penetrator missions to asteroids and comets to be the next level study for the primitive bodies. However, there was general agreement that the most important scientific step following the rendezvous mission(s) to comets and asteroids would be the return of a cold comet sample. Only by having a preserved cold sample available for laboratory analyses will we be able to learn the nature of the mixing of the dust and ice, the structure and chemical composition of the ices, and the small-scale stratigraphy that may have resulted from accretion. Laboratory samples will be necessary to search for and identify the nature of any presolar or interstellar dust that may be trapped in comets. Any age-dating studies will also require samples for laboratory analysis. Study of a pristine sample of a comet is probably the only way to extend our knowledge in detail of the chemical, isotopic, and mineralogic conditions beyond the limits of our solar system in both space and time.

The return of a well-preserved, well-characterized sample of a comet nucleus was not studied in detail by the study group but has been studied by Science Applications Incorporated for NASA. The mission requires Shuttle launches and SEP or NEP (nuclearelectric propulsion). Round trip times are typically 5 to 7 years. The planned sampling scenario involves the retrieval of two cores approximately 148 centimeters in diameter and 1 meter in length. An instrument payload similar to that envisioned for rendezvous missions would be carried on the parent spacecraft to characterize the comet nucleus and select sampling areas. Remote sampling devices deployed from the parent spacecraft would carry out limited in situ characterization of the sampled area. Preliminary study indicates that passive cooling and insulation techniques would be sufficient to preserve the sample at or below $110 \mathrm{~K}$, assuming an initial temperature of $100 \mathrm{~K}$. 


\section{COMET TARGET SELECTION, by M. Festou and D. K. Yeomans}

The list of possible target comets is presented in Table C.8. In that table the comet's name is followed by its minimum magnitude, its perihelion distance in $\mathrm{AU}$, its orbital inclination, number of observed returns, date of perihelion passage and the ground-based observing conditions of the comet when it is near perihelion. Only comets with a perihelion magnitude brighter than 12 are included. Comet Halley is included for comparison.

Some comets are notorious for their peculiar behavior: $\mathrm{P} /$ Encke is always brighter pre-perihelion and fades quickly after its closest approach to the sun. P/d'Arrest and P/Tempel 2 act in the opposite way: they remain relatively inactive until perihelion and then brighten during a few weeks as if their heliocentric distance were decreasing instead of increasing. Comet $P /$ Tuttle-Giacobini-Kresak has a very erratic nucleus activity (two 9 magnitude flares were observed in 1973), and it would become a first priority target if it could gratify us with such a show at each of its appearances, but this is not the case. Comet $\mathrm{P} /$ Wild 2 is unique, as will be seen more quantitatively later on. It has only been seen once after its capture by Jupiter in the mid-seventies (International Astronomical Union (IAU) Circular 3167) and, due to unfortunate viewing conditions in 1978 , it was only seen prior to perihelion passage.

The gas production in comets as a function of the heliocentric distance varies according to the following law:

$$
Q_{g a \theta}=Q_{o} r_{H} n
$$

The index $\mathrm{n}$ might depend on $r_{H}$ and be different at the same $r_{H}$ before and after perihelion. The detection of parent molecules for a rendezvous near perihelion will depend upon the comet's ability to produce gas at or near its perihelion. However, the spatial extension of the parent molecule cloud is roughly proportional to the lifetime of the parent species. Hence, for two comets that have the same gas production rate at their respective perihelia, 


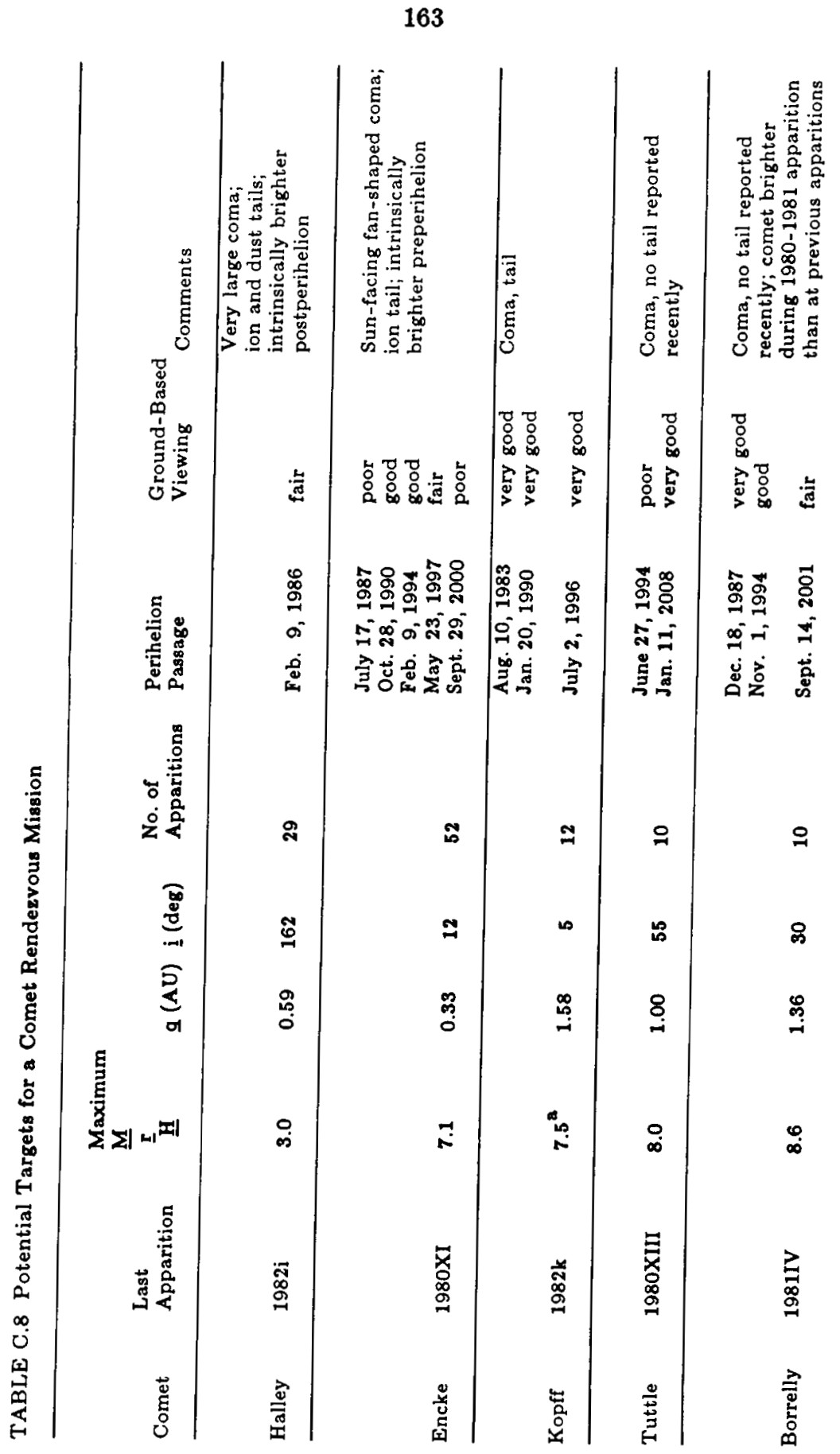




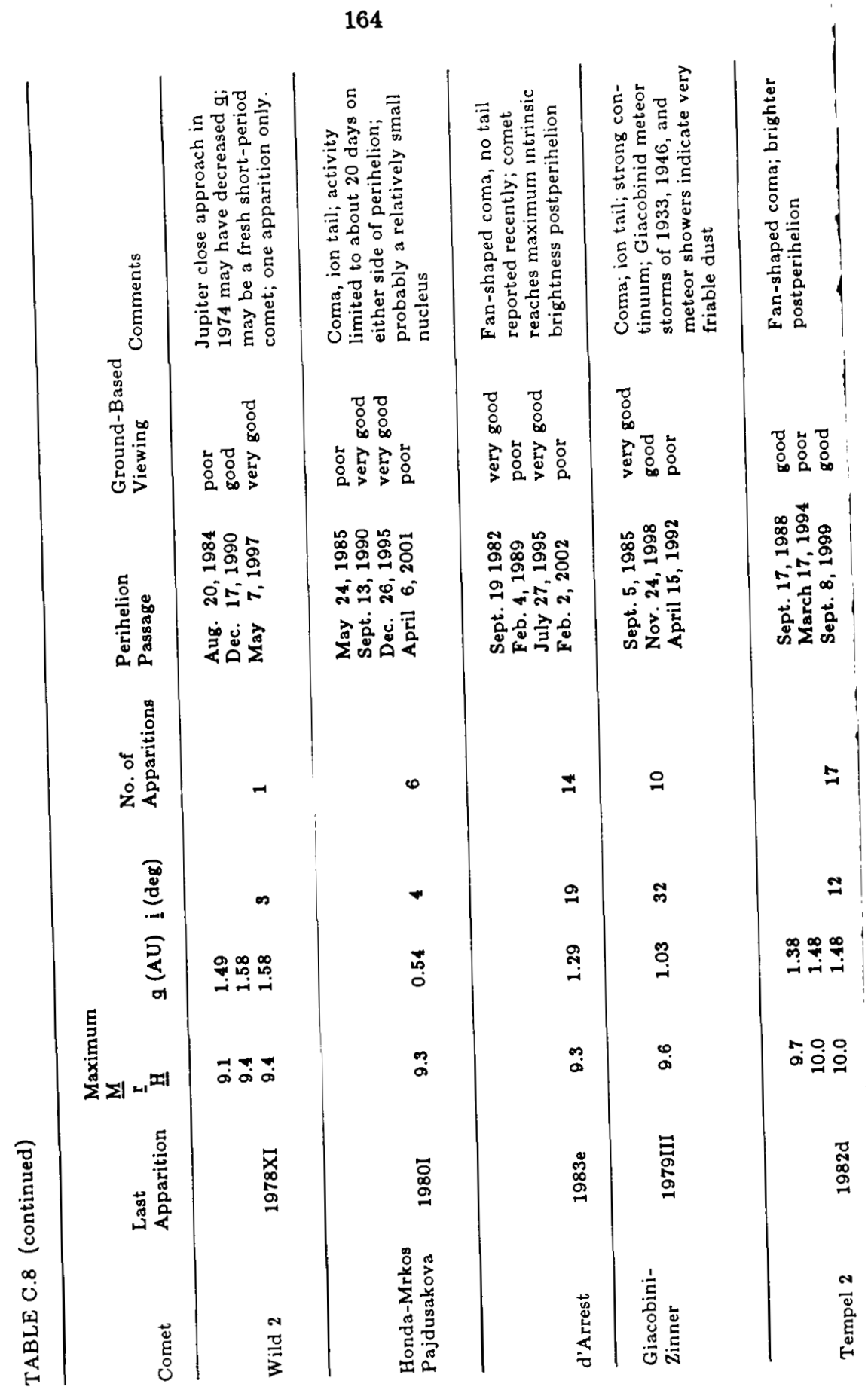




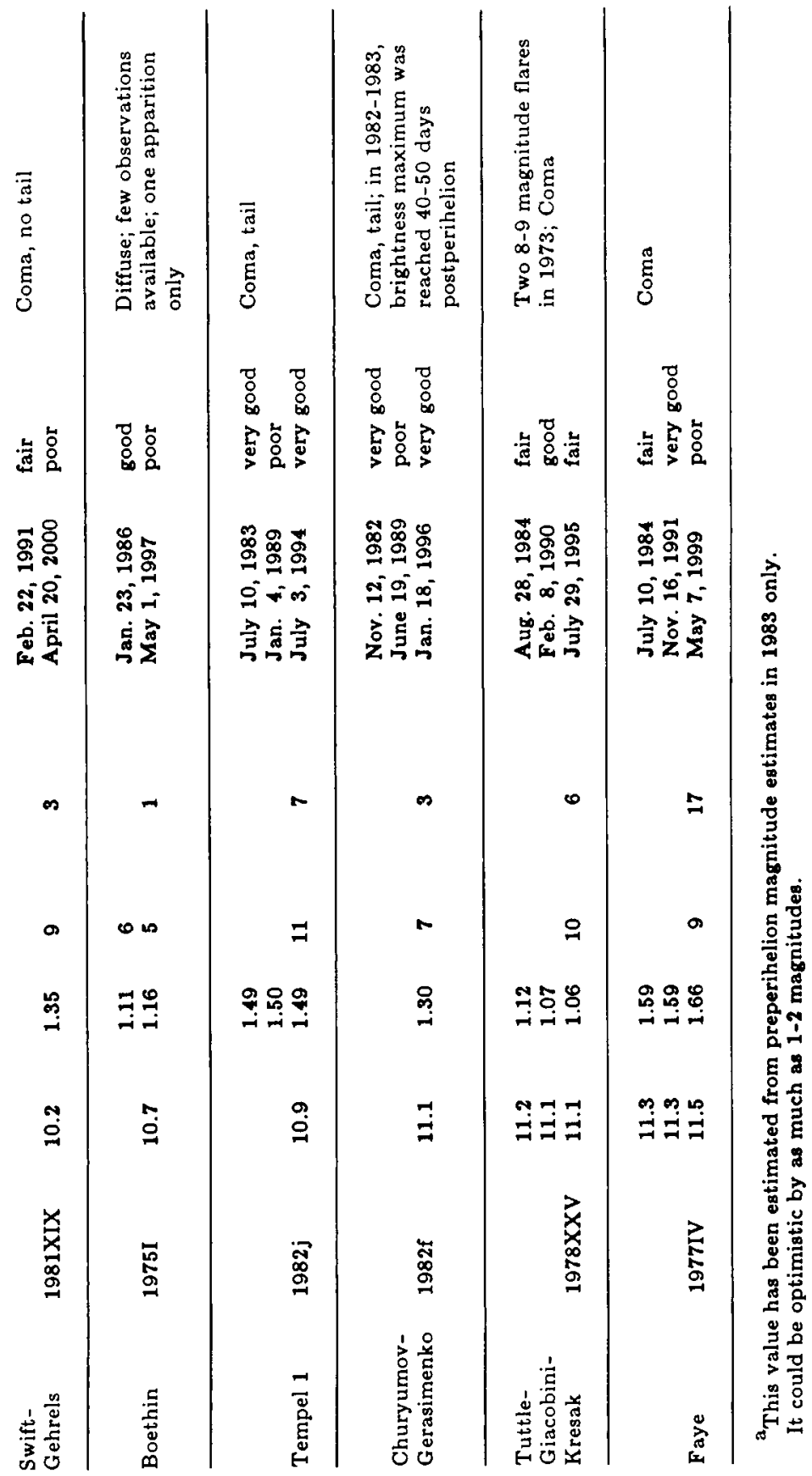


the comet with the larger perihelion distance is to be preferred for the detection of parent molecules.

The detection of daughter and granddaughter products not only depends on the total gas production but also upon the photodissociation rate. The highest daughter species densities will be reached close to the sun. The mean daughter product density (over the entire coma) varies as $r_{H}^{n-4}$ : since the exciting solar flux varies as $r_{H}{ }^{-2}$, one understands that a "small" comet (such as Comet P/Encke; see below) could become a fairly bright object without producing much gas.

The study of ions is facilitated when the ionization is at its maximum, i.e., near the sun. However, gas phase reactions mainly depend on the collision frequency, i.e., on the total gas production. The interaction of the coma with the solar wind is certainly higher at small heliocentric distances.

The nucleus might be easily seen, prior to perihelion, in a comet whose activity peaks at or after perihelion passage. The reverse situation may not obtain since, even if the nucleus were inactive, it still could be surrounded by a cloud of particles released a few weeks earlier.

In order to estimate the gas production as a function of time from perihelion of the various comets, the following method was used:

- A set of comets both well observed from the ground and from the IUE spacecraft was selected.

- Only nondusty comets were considered, so that visual magnitudes represent the total content of the coma in $\mathrm{C}_{2}$ radicals.

- It was assumed that the productions of $\mathrm{C}_{2}$ and $\mathrm{OH}$ radicals are proportional and that their ratio does not vary from comet to comet.

Thus, using absolute measurements made by IUE and using an appropriate coma model, it was possible to relate visual magnitudes and $\mathrm{OH}$ gas production. This basic assumption results from recent IUE measurements by Festou, Feldman, A'Hearn, and co-workers on the composition of coma species emitting in the UV.

The magnitude of a comet can be written in the form

$$
M_{T}\left(\Delta, r_{H}\right)=5 \log \Delta+M\left(r_{H}\right)
$$

where the term $M\left(\mathrm{r}_{H}\right)$ is the "heliocentric magnitude." It only depends on $r_{H}$. Visual observations of the eight comets were 


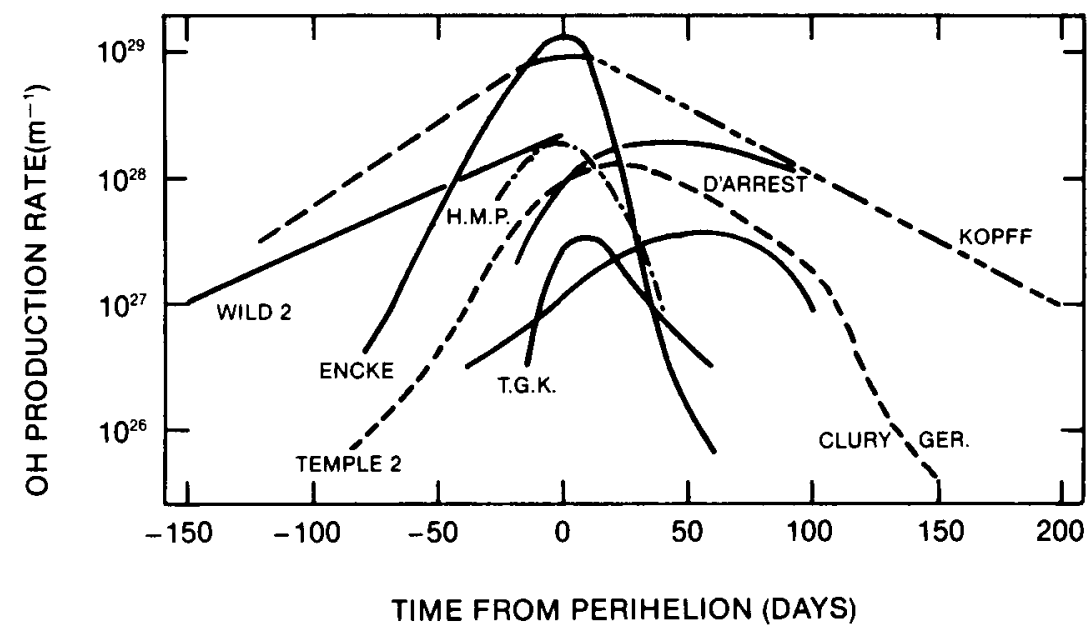

FIGURE C.2 Gas production rates versus time from perihelion.

reduced to determine $M\left(r_{H}\right)$. The relation between this parameter and the $O H$ production rate $Q_{O H}$ is

$$
\log \left[Q_{O H}\left(r_{H}\right)\right]=32.0-0.4 M\left(r_{H}\right) \pm 0.2
$$

Values of the gas production rates for the candidate comets are presented in Figure C.2. For the indicated days before and after perihelion, the gas production rates are given in units of $\mathrm{OH}$ molecules per second. When the curve is highly asymmetrical, this indicates that only one fraction of the nucleus is ice covered. For example, this fraction is directed toward the sun at perihelion if the maximum occurs at perihelion. The "width" of the light curves depends on both the orbital parameters of the comet and the value of the fraction of the nucleus surface that is exposed to the sun's heat. Two families of comets appear:

1. In the first one, the gas production is mainly governed by the heliocentric distance of the comet, whatever its subliming area value (Encke, Honda-Mrkos-Pajdusakova, Wild, and Kopff).

2. In the second one, the gas production is mainly determined by geometric effects, i.e., angle between the radius vector and the rotation axis (Tuttle-Giacobini-Kresak, Churyumov-Gerasimenko, d'Arrest, and Tempel 2). 
Preferred Targets If rendezvous is achieved close to (and prior to) perihelion, observation of periodic comets d'Arrest, Tempel 2, Tuttle-Giacobini-Kresak, and Churyumov-Gerasimenko offers a good chance to observe a bare or only partially covered nucleus and then the development of the coma. Due to its fairly high gas production rate, $\mathrm{P} /$ Tempel 2 is the best choice (too high an inclination for $\mathrm{P} / \mathrm{d}$ 'Arrest).

Comet $\mathrm{P} /$ Encke would be a better choice because of its low perihelion value (which allows ion chemistry to develop) if it were not so costly in terms of the energy required for a spacecraft rendezvous.

The two comets that best resemble new comets are certainly $\mathrm{P} / \mathrm{Kopff}$ and $\mathrm{P} /$ Wild 2. The first one is the best choice, since it is active over a long period of time and it has a much longer observational history. However, if the activity of $\mathrm{P} / \mathrm{Wild} 2$ were confirmed during the next apparition, it would become a good candidate also. 


\section{SOLAR-ELECTRIC PROPULSION, by H. Loeb and E. Stuhlinger}

The possibility of electric propulsion systems for spacecraft was mentioned by the early pioneers of spaceflight: Tsiolkovskii, Goddard, and Oberth. The first study proving the technical feasibility and the substantial usefulness of "ion propulsion systems" on planetary and interplanetary missions was published in 1954. Development work on several types of electric propulsion devices began in 1958 in the United States and around 1960 in Europe. It concentrated on "ion thrusters" and "plasma thrusters." While both types proved to be feasible, ion thrusters assumed and maintained the leading position because of their higher efficiency and their longer service life expectancy.

Electric propulsion systems, in contrast to chemical propulsion systems, require a separate source of energy. Two types of energy sources have been considered, solar-electric and nuclear-electric power supplies. Considerable experience on Earth and in space exists already for solar-electric generators. Nuclear-electric power sources, incorporating a uranium fission reactor, have been studied theoretically and experimentally, but no such system has been built as yet in the United States or in Europe.

Table C.9 compares the basic features of chemical and electric propulsion systems and shows some of the fundamental relations that govern the performance of the two systems. A schematic diagram of an ion thruster is shown in Figure C.3. Many different concepts of thruster design were studied and tried out during the past 25 years in all countries that have been actively involved in space flight projects. The two leading contenders among ion thrusters, both of which have reached a high degree of development, are the Kaufman thruster, developed at the NASA Lewis Research Center and built by the Hughes Research Corporation in Los Angeles, and the Loeb thruster, developed and built at the University of Giessen. Some models of the Loeb type were built and tested at Messerschmitt-Boelkow-Blohm (MBB) in Munich.

Loeb thrusters have been tested extensively in the laboratory but not yet in space. One of the Loeb thrusters, RIT 10, was 
TABLE C.9 Basic Features of Chemical and Electric Propulsion Systems

\begin{tabular}{llllll}
\hline & $\begin{array}{l}\underline{v}_{\mathrm{EX}} \\
(\mathrm{m} / \mathrm{s})\end{array}$ & $\begin{array}{l}\text { Acceleration } \\
\left(\mathrm{m} / \mathrm{s}^{2}\right)\end{array}$ & $\begin{array}{l}\text { Propulsion } \\
\text { Time }\end{array}$ & $\begin{array}{l}\text { Terminal } \\
\text { Velocity } \\
(\mathrm{km} / \mathrm{s})\end{array}$ & $\begin{array}{l}\text { Payload } \\
(\%)\end{array}$ \\
\hline Chemical rocket & 4,400 & 40 & Minutes & $6-8$ & $1-2$ \\
Electric rocket & 35,000 & $10^{-3}$ & $1-2$ years & $15-20$ & 10 \\
\hline
\end{tabular}

built, tested, and fully space qualified for the attitude and position control system of a television satellite; however, it has not yet been used in such a capacity.

Several years ago, the National Aeronautics and Space Administration (NASA) began simultaneous development of 8-centimeter-diameter and 30-centimeter-diameter mercury ion engines, the 8-centimeter for auxiliary propulsion applications such as north/

south station keeping and the 30 centimeter for primary propulsion applications such as interplanetary travel. The results of this effort have been (1) the flight qualification of the 8-centimeter engine in a program called IAPS (Ion Auxiliary Propulsion System), and (2) the prototype qualification of the 30-centimeter design for the solar-electric propulsion system (SEPS) program.

Two 8-centimeter mercury ion engines are integral components of an IAPS flight package that will test engine capability to perform 7 years of north/south station keeping and that will characterize the thruster plume. IAPS will be flown on an Air Force Space Test Program satellite. The engines have been flight qualified and are presently installed on the P80-1 spacecraft undergoing integration tests.

Spacecraft equipped with electric propulsion systems are distinguished by relatively large payload capabilities ( 5 to 10 percent of the total initial spacecraft mass), by substantial velocity gains during operation ( 15 to $20 \mathrm{~km} / \mathrm{s}$ ), and by a considerable flexibility in the layout and conduction of a specific mission that results in relatively wide launch and target windows for any given mission.

On the other hand, electrically propelled spacecraft are capable of accelerations of only $10^{-4}$ to $10^{-3} \mathrm{~m} \mathrm{~s}^{-2}$. They have to begin 


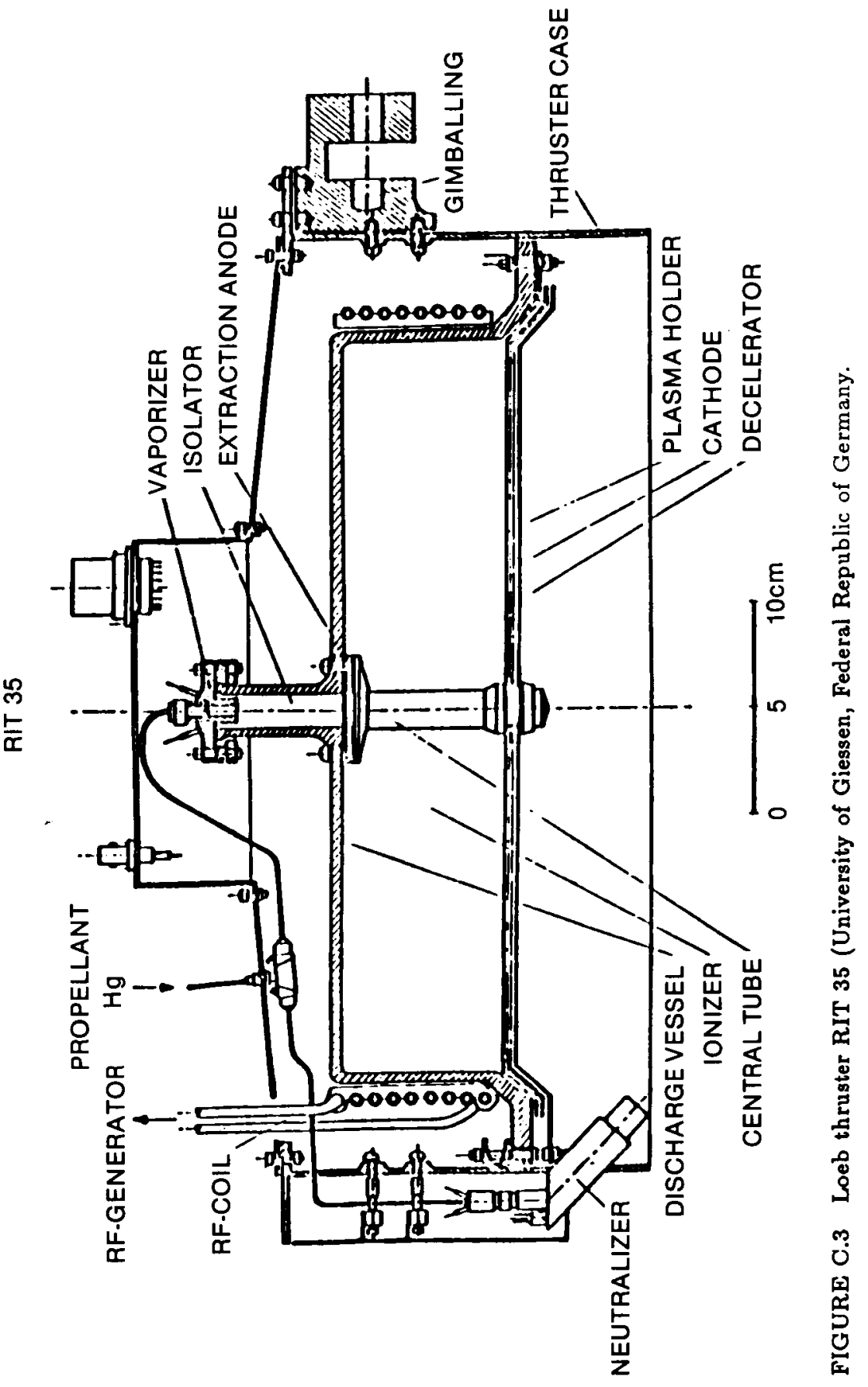


their powered flight after an initial boost by a powerful launcher, such as Ariane IV or Shuttle-Centaur, and they will obtain the full benefit of their potentially high velocities only on missions that cover several years in space. There are, however, a number of different types of flight missions for which electric propulsion systems would be ideally suited: comet and asteroid rendezvous, comet sample return, asteroid sample return, terrestrial planet orbiters and landers, Phobos and Deimos sample return, out-ofecliptic probe, and long baseline radioastronomy probe. Several of these missions, because of their large velocity requirements, can be executed with a reasonably high payload and the Shuttle-Centaur as a launcher only if the spacecraft is equipped with an electric propulsion system. Among these missions are multiple rendezvous missions to comets and asteroids, and pristine sample collection missions to comets with a "soft" return to Earth. An electrically propelled spacecraft for such missions consists of the following basic components:

- solar-electric power source,

- ion thrusters with power conditioning units,

- spacecraft structure with electric wiring harness,

- attitude control system,

- guidance and control system,

- data and communication system,

- scientific instrumentation,

- thermal control system,

- adapter for Centaur stage, and

- cradle to hold spacecraft in Shuttle bay.

The first three of these components, representing the "solarelectric propulsion" or SEP system, require a very intimate electric and mechanical integration. They should be developed, built, and tested as a unit under the auspices of the same source, while the other components can be built and added to the spacecraft as independent modules. In a joint NASA-European Space Agency (ESA) project for a mission to comets and asteroids, the SEP system could be contributed either by NASA (Kaufman thruster) or by ESA (Loeb thruster). While at present the 30-centimeter Kaufman thruster is definitely closer to the state of a production model than the 35-centimeter Loeb thruster, both thrusters in their present states would require further development work to give them the optimum features for a comet-asteroid rendezvous 
mission as it is considered here. This is particularly true for the power conditioning units; both the U.S. and the European versions would have to be redesigned to incorporate modern lightweight components.

The solar-electric power source of the SEP system would consist of two flat arrays, without concentrators. The system would be laid out for $54 \mathrm{kWe}$ of total power at $1 \mathrm{AU}$; however, at solar distances less than 1.5 AU, the arrays would be turned into an oblique direction with respect to the sun so that the actual power output would never exceed $32 \mathrm{~kW}$. The electric power, which is always proportional to the thrust, as a function of solar distance is shown in Figure C.4. This function includes the effect of increasing solar cell efficiency with decreasing temperature. Beyond a distance of about 2.5 AU, the solar intensity is so low that the cell temperature drops to a level where cell operation begins to be irregular. For that reason, the thruster system will not be operated at solar distances beyond $2.5 \mathrm{AU}$.

Two types of SEP missions have been considered so far: (1) a multitarget mission incorporating rendezvous encounters with one comet and two to three asteroids, and (2) a mission to one comet with "soft" return of a sample of pristine coma material to Earth orbit. In each case, total mission time would not exceed about 5.5 years. The total operating time for any one of the seven thrusters would be less than 18,000 hours. Each of the missions would use the same basic design of electric propulsion system, solar-electric power supply, data and communications system, guidance and control equipment, and spacecraft structure. The missions would differ with respect to amount of propellant, total mission time, thrusting sequence, and assortment of scientific instruments.

A rough time schedule for any one of these missions is shown in Figure C.5.

The SEP system as described here is summarized in Table C.10. It can be scaled down easily to accommodate smaller payloads and less powerful launchers. If the Ariane IV were chosen for the launching, the SEP configuration would incorporate only four or five thrusters, the payload would be reduced, and only one or two targets would be selected for rendezvous encounters during one flight mission. However, design and operation of the ion thrusters, of the solar-electric power source, and of the other spacecraft components would be basically the same as described for the multitarget missions. 


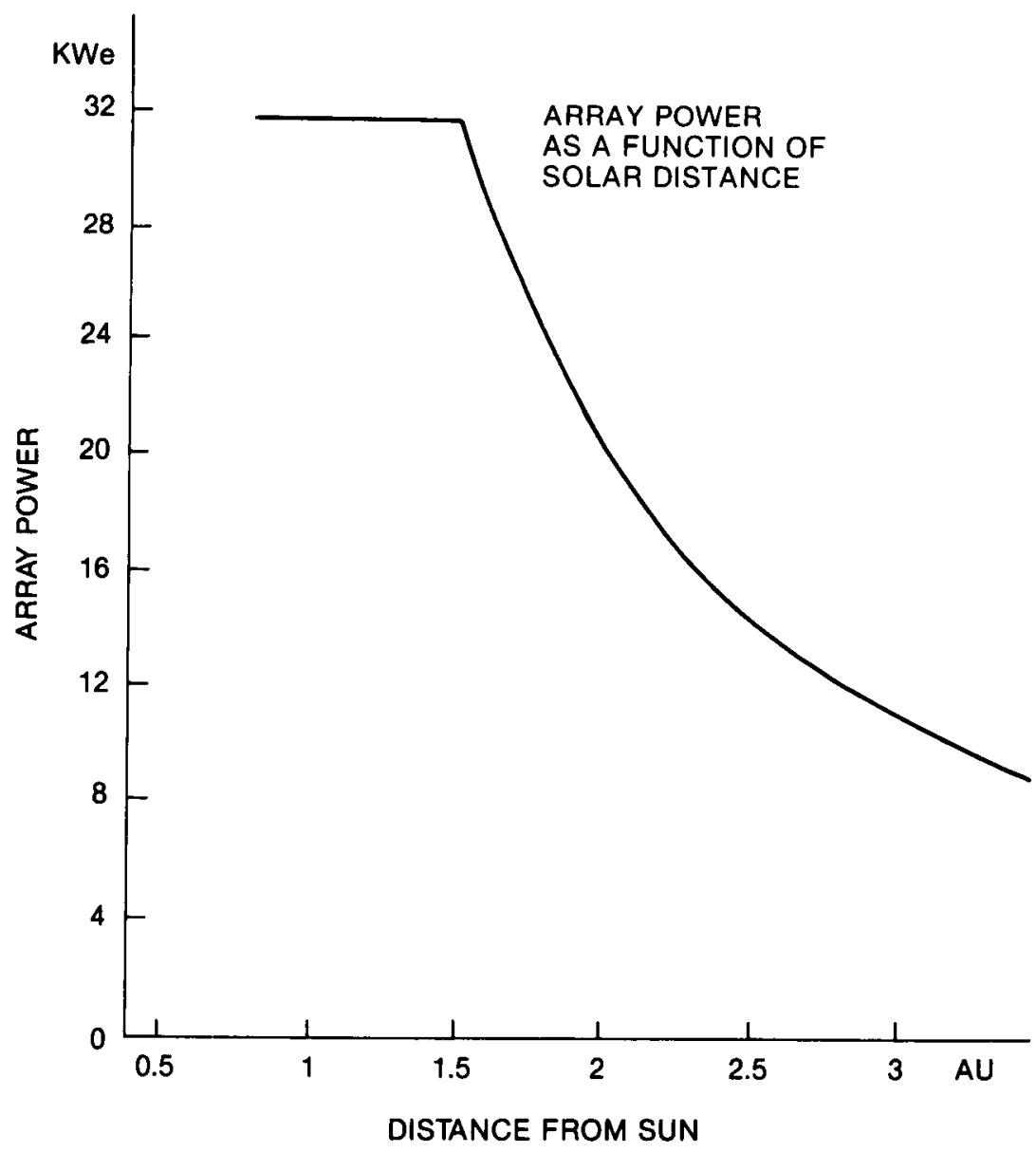

FIGURE C.4 Electric power output of solar-electric array as a function of solar distance. 
TABLE C.10 Characteristics of Proposed SEP-Driven Spacecraft for Primitive Body Missions

\begin{tabular}{ll}
$\begin{array}{l}\text { Spacecraft Mass (total after } \\
\text { Centaur separation) }\end{array}$ & $4000 \mathrm{~kg}$ \\
Electric power (at 1-1.5 AU) & $32 \mathrm{~kW}$ \\
Number of thrusters & $7-8$ \\
Propulsion time per thruster & 2 years \\
Solar cell area & $360 \mathrm{~m}^{2}$ \\
Propellant mass & $1,600-1,800$ \\
Science payload & $100-300 \mathrm{~kg}$ \\
Launcher & Shuttle plus Centaur \\
\hline
\end{tabular}

83

Test and Qualification

Fabrication and Testing 


\section{INSTRUMENTATION FOR COMET AND ASTEROID MISSIONS}

The instruments described in this section are examples. In some cases there are several different instruments that could be grouped under the generic titles listed in the example payloads (Tables C.4 and C.6).

Some of the instruments have been flown, others require considerable development. The purpose of including this section is to illustrate the rich array of scientific instruments potentially available to address the scientific objectives for comet and asteroid missions.

The instruments appear in roughly the order in which they are listed in Tables C.4 and C.6. 


\section{Camera}

SCIENTIFIC OBJECTIVE:

The camera should be designed to assess the visible morphology of an asteroid, comet nucleus, and cometary envelope by imaging the regions of interest at a variety of resolutions and through a variety of spectral filters in the visible and near infrared. Imaging provides the basic information necessary for assessing physical, structural, and geological characteristics of solid objects and for recording the changing configuration of evolving parts of comets such as the cometary atmosphere. The photometric and spectrophotometric measurements at various places also yield the composition and microstructure of comets and asteroids.

OPERATING PRINCIPLE:

Camera optics and filters, exposure and control system, solid state detector, and signal read-out

PRIMARY DATA:

Two-dimensional images

HERITAGE:

Galileo, Giotto, etc.

DEVELOPMENT STATUS:

Existing hardware for various spacecraft 


\section{Infrared Mapping Spectrometer}

SCIENTIFIC OBJECTIVE:

The Infrared Mapping Spectrometer provides detailed mapping of the mineralogy over the surface, including identification of common ices. In an asteroid flyby, this instrument may provide the only information on surface composition. For either comets or asteroids, the high spatial resolution obtainable from a spacecraft will permit an analysis of the heterogeneous nature of the surface, thereby illuminating the accretional and geological history of the object. An additional scientific objective of an infrared instrument would be the measurement of the surface temperature and mapping of the temperature distribution. In the case of the asteroids, the variation of temperature with insulation will provide information on the thermophysical properties of the upper few centimeters of the surface, while similar measurements of surface temperature for a cometary nucleus will permit a determination of the surface energy balance and an estimate of the rate of loss of volatiles at various surface locations.

OPERATING PRINCIPLE:

Several separate instruments could be used to accomplish the scientific objectives summarized above. These might include, for instance, a visible-IR spectrometer ( $300 \mathrm{~nm}$ to 5 micrometers), a mapping or imaging IR device, and a thermal infrared radiometer ( 5 to 50 micrometers).

The basic instrument is an optical-IR imaging spectrometer. It consists of a telescope with $15 \mathrm{~cm}$ aperture feeding a group of spectral dispersing elements and detector arrays, optimized for each wavelength interval. An additional optical feed brings nondispersed thermal radiation to a filter-wheel radiometer. With rapid current development of optical and infrared detector arrays, including a new generation of large two-dimensional charge-coupled device (CCD) detectors, it is anticipated that any detectors specified now will be supplanted by superior designs by the time a specific mission is initiated.

INSTRUMENT SPECIFICATION :

Wavelength interval: $300 \mathrm{~nm}$ to 5 micrometers, plus thermal Spectral resolving power: 100

Field of view: $1.0-0.1$ milliradian

Telescope: $15 \mathrm{~cm}$ aperture, $\mathrm{f} / 10$

Weight: $10-12 \mathrm{~kg}$ 
Power: 5-10 watts

Data rate: maximum a few kbps

HERITAGE:

Galileo

DEVELOPMENT STATUS:

Flight (NIMS); breadboard

SUPPORTING SYSTEMS:

Scan platform

Possible passive radiators to cool detectors 


\section{Neutral Mass Spectrometer}

SCIENTIFIC OBJECTIVE:

The neutral mass spectrometer will be used for (1) determination of the chemical, elemental, and isotopic composition of the volatile components of the cometary material, (2) investigation of the physics and chemistry of the cometary atmosphere and ionosphere at different solar distances, and (3) determination of the nature of cometary interactions with solar wind and solar radiation.

OPERATING PRINCIPLE:

Mass spectrometer using multiple ionization processes (i.e., electron impact, field ionization) with magnetic/electric field analyzer or time-of-flight instrument for mass analysis.

PRIMARY DATA:

Mass spectra

Data range: $100 \mathrm{bps}$ (minimum) - 2,000 bps (maximum)

HERITAGE:

Pioneer Venus

Dynamic Explorer

Giotto (electron impact ionization source plus magnetic/electric field analyzer)

VEGA (field ionization source plus time-of-flight technique)

DEVELOPMENT STATUS:

Almost flight ready for Giotto and VEGA missions to Comet Halley for first in situ measurements of cometary neutral atmosphere. Further development required for low-density environment $(n=$ $10^{3} \mathrm{~cm}^{-3}$ ) and high-velocity fragments from photodissociation and charge exchange process.

INSTRUMENT SPECIFICATION:

Mass range: 1-150 atomic mass units (AMU) (with extension to higher mass range for aromatic hydrocarbons and other complex molecules desirable)

Detection threshold: $10^{3} \mathrm{~cm}^{-3}$

Dynamic range: $10^{6}$

Resolution: $10^{-4}$ at adjacent half mass

Weight: $10 \mathrm{~kg}$

Power: $20 \mathrm{~W}$

SUPPORTING SUBSYSTEMS:

None 


\section{Alpha-Proton Backscatter}

SCIENTIFIC OBJECTIVE:

Alpha-proton backscatter devices will be used for elemental analysis of major and minor elements in a 50 to 500 microgram $/ \mathrm{cm}^{2}$ layer of collected dust. The experimenters predict detection of 99 percent of the atoms in the sample (excluding hydrogen) with a precision of 1 percent for selected elements. In combination with $x$-ray excitation, detection levels in the parts-per-million range will be possible for certain elements.

OPERATING PRINCIPLE:

Measures the energy spectrum of protons and alpha particles backscattered from the collected sample.

\section{PRIMARY DATA:}

Elemental composition

HERITAGE:

Surveyor; prototypes developed for Viking's lander and penetrators

DEVELOPMENT STATUS:

Advanced state of development but needs adaptation to a particular mission. Additional development is needed on the alpha source.

INSTRUMENT SPECIFICATION:

Mass: $1 \mathrm{~kg}$

Power: $2 \mathrm{~W}$

SUPPORTING SUBSYSTEMS:

Sample collector and possible cooling for detectors if $\mathrm{x}$-ray analysis is included. 


\section{X-Ray Fluorescence}

SCIENTIFIC OBJECTIVE:

$\mathrm{X}$-ray fluorescence will provide elemental analysis of major and minor elements in a bulk sample of 10 to 100 micrograms $/ \mathrm{cm}^{2}$ of collected dust. For a chondritic composition sample, it may be possible to provide meaningful analyses for $\mathrm{Mg}, \mathrm{Si}, \mathrm{S}, \mathrm{K}, \mathrm{Ca}$, $\mathrm{Ti}, \mathrm{Cr}, \mathrm{Mn}, \mathrm{Fe}, \mathrm{Ni}, \mathrm{P}, \mathrm{Cl}, \mathrm{Zn}, \mathrm{Sr}, \mathrm{Y}, \mathrm{Zr}$, and possibly $\mathrm{C}, \mathrm{N}$, and $\mathrm{O}$. The instrument could incorporate an alpha backscatter system to provide good light element sensitivity. Incorporation of an electron spectrometer to analyze the energy of photoelectrons might, in addition, provide useful information on the chemical state of the light elements. It has been suggested that with a cooled collection substrate, the combined instrument could also analyze a condensed or absorbed sample of coma gas.

OPERATING PRINCIPLE:

Measures the $x$-ray spectrum of a collected dust sample excited by an on-board $x$-ray source

PRIMARY DATA:

Elemental composition of a collected dust sample

HERITAGE:

Viking and Venera

DEVELOPMENT STATUS:

Advanced development except for sensor miniaturization and cooling system

INSTRUMENT SPECIFICATION:

Mass: $6 \mathrm{~kg}$

Power: $4 \mathrm{~W}$

Data rate: $0.1 \mathrm{kbps}$

SUPPORTING SUBSYSTEMS:

Dust collector and cryogenic cooler 


\section{Secondary Ion Mass Spectrometer/ \\ Laser Ionization Mass Spectrometer}

SCIENTIFIC OBJECTIVE:

These instruments will determine the bulk elemental and isotopic composition of a collected dust sample.

OPERATING PRINCIPLE:

In the combined instrument, ions are produced by sputtering with an ion beam and by pulsed heating with a laser beam. The ion spectrum gives the average composition of the area of the sample hit by the laser or ion beam. Composition changes during sputtering can give depth information useful for detecting core-mantle grains composed of low $\mathrm{Z}$ mantles on high $\mathrm{Z}$ cores.

PRIMARY DATA:

Elemental and isotopic composition of a collected comet dust sample

HERITAGE:

New instrument

DEVELOPMENT STATUS:

Under study for comet missions

INSTRUMENT SPECIFICATION:

Mass: $10 \mathrm{~kg}$

Power: $50 \mathrm{~W}$

SUPPORTING SUBSYSTEMS :

Dust collector 


\section{Scanning Microscope/X-Ray Analyzer}

SCIENTIFIC OBJECTIVE:

This instrument will perform high-resolution SEM imaging and elemental composition for at least several hundred collected grains in the 0.2 micron and larger size range. The relative accuracy for elemental determination is 3-10 percent for many major and minor elements.

OPERATING PRINCIPLE:

Collected dust is located and imaged with a scanning electron beam. Fluorescence $x$-rays are detected by an energy dispersive detector.

PRIMARY DATA:

SEM images and elemental composition of a thousand individual dust grains in the 0.2 micron and larger size range

HERITAGE:

New instrument

DEVELOPMENT STATUS:

A working breadboard has been developed INSTRUMENT SPECIFICATION:

Mass: $10 \mathrm{~kg}$

Power: $28 \mathrm{~W}$

SUPPORTING SUBSYSTEMS:

Cooling may be needed for $\mathrm{x}$-ray detector, dust collector 
Gas Chromatograph/Mass Spectrometer

SCIENTIFIC OBJECTIVE:

This instrument will determine the abundance, molecular form, and isotopic composition of the volatile (organic, etc.) fraction of collected cometary solids.

OPERATING PRINCIPLE:

The volatilized sample is passed through a multicolumn gas chromatograph and an in-series mass spectrometer.

PRIMARY DATA:

The molecular forms of the light elements $\mathrm{C}, \mathrm{H}, \mathrm{N}, \mathrm{S}, \mathrm{O}$, and $\mathrm{P}$

HERITAGE:

Viking and Pioneer Venus

DEVELOPMENT STATUS:

Modest development activities remain to be conducted prior to construction of a design verification unit. A method must be developed to collect and concentrate an adequate amount of sample. Development is continuing of a gas chromatograph on-a-chip produced by silicon lithography techniques.

INSTRUMENT SPECIFICATIONS:

Mass: $18 \mathrm{~kg}$ (conventional system)

Power: $45 \mathrm{~W}$

SUPPORTING SUBSYSTEMS:

Sample collector/concentrator 
Isotope Dilution Mass Spectrometer

SCIENTIFIC OBJECTIVE:

This instrument will determine accurate elemental and isotopic abundances for selected elements in a collected cometary dust sample.

OPERATING PRINCIPLE:

The collected sample is dissolved, spiked with material of known isotopic composition, loaded on a thermal ionization filament, and run in a solid source mass spectrometer.

PRIMARY DATA:

Elemental and isotopic composition of a collected dust sample HERITAGE:

A new instrument

DEVELOPMENT STATUS:

That was under study for the mid-1980s Tempel 2 mission. INSTRUMENT SPECIFICATION:

To be determined SUPPORTING SUBSYSTEMS :

Dust sample collector 


\section{Ion Mass Spectrometer}

SCIENTIFIC OBJECTIVE:

The ion mass spectrometer will be used for (1) determination of the chemical, elemental, and isotopic composition of the cometary ions, (2) determination of the three-dimensional velocity distribution of solar wind and cometary plasmas in the vicinity of the comet, and (3) determination of the nature of comet-solar wind interaction at different solar distances.

OPERATING PRINCIPLE:

Electrostatic analyzer plus magnetic sector for ions in the energy range $0-10 \mathrm{KeV}$

Electrostatic analyzer plus time-of-flight instrument for ions of higher energies (10-100 KeV). Energy spectra are obtained by variable preacceleration/deceleration.

PRIMARY DATA:

Ion mass (1-65 AMU or higher), elevation and azimuth angles and incidence energy (three-dimensional velocity distribution and ion composition).

HERITAGE:

GEOS, Giotto, and ISEE

DEVELOPMENT STATUS:

Ion mass spectrometer with electrostatic analyzer/magnetic filter and implanted ion sensor with electrostatic analyzer/time-of-flight analyzer are near completion for the Giotto mission to comet Halley. Development to acquire wide field-of-view in a three-axis stabilized spacecraft is needed.

INSTRUMENT SPECIFICATION :

Ion mass spectrometer

Mass: $8 \mathrm{~kg}, 10 \mathrm{~W}$ (maximum), 1,000 bps

Implanted in sensor

Mass: $3 \mathrm{~kg}, 3 \mathrm{~W}, 500 \mathrm{bps}$

SUPPORTING SUBSYSTEMS:

None

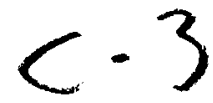




\section{Millimeter Wave Radiometer}

SCIENTIFIC OBJECTIVES:

The millimeter wave radiometer will measure nucleus surface and subsurface properties such as temperature, temperature gradient, composition, etc. A spectrometer mode would allow measurement of gaseous constituents in vicinity of nucleus.

OPERATING PRINCIPLE:

Radiometer/spectrometer operating at $6.9,3.4$, and $1.7 \mathrm{~mm}$ DEVELOPMENT STATUS :

Millimeter wave radiometers in all wavelength ranges have been developed. However, at shorter wavelengths, radiometers have not been used in space missions. Dichroic filters, feeds, and $1.7 \mathrm{~mm}$ harmonic mifer need additional development.

INSTRUMENT SPECIFICATIONS:

Size: $0.006 \mathrm{~m}^{3}$ in three packages

Weight: $11 \mathrm{~kg}$

Power: (duty cycle dependent): $5 \mathrm{~W}$ for 10 percent duty cycle SUPPORTING SUBSYSTEMS:

Parabolic antenna on pointing platform; antenna to have surface accuracies sufficient to support $1.7 \mathrm{~mm}$ observations 


\section{EUV Spectrophotometer}

SCIENTIFIC OBJECTIVE:

The extreme ultraviolet (EUV) spectrophotometer will be used for measurement of coma composition in its elemental $(\mathrm{C}, \mathrm{H}, \mathrm{O}, \mathrm{N}$, $\mathrm{C}^{+}, \mathrm{O}^{+}$) form, study of photochemical processes, measurement of UV reflectivity of dust particles, monitoring of the activity of the nucleus (short-lived species), search for rare gases, and absorption studies.

OPERATING PRINCIPLE:

Remote sensing mode: mapping of all emissions having spatial extensions up to $\sim 300,000 \mathrm{~km}$ in diameter

In situ mode: deconvolution of extended emissions by moving the probe inside the coma; detailed mapping of weak emissions concentrated toward the nucleus $(\sim 30,000 \mathrm{~km}$ or less in diameter); occultation experiments

INSTRUMENT SPECIFICATION:

The new capabilities of imaging CCD spectrographs are not very well known but seem very encouraging. The spectrophotometer should cover the $500-2,000 \AA$ range with a 3-5 $\AA$ spectral resolution. Spatial resolution during remote viewing (RV) phase of about $19 \mathrm{~km}$ at closest approach is desirable. 


\section{Near UV-Visible Spectrophotometer}

SCIENTIFIC OBJECTIVE:

The near UV-visible spectrophotometer will assess composition of the coma (mainly radicals and ions), will study photochemical processes, ion creation processes, and interaction of ions with the solar wind, will monitor the activity of the nucleus (short-lived species, forbidden oxygen lines), the optical reflectivity of dust particles, and the optical depth in the continuum.

OPERATING PRINCIPLE:

As for the EUV spectrophotometer, two operating modes should be used allowing remote sensing studies as well as in situ measurements. The use of various viewing geometries should yield to a deconvolution of the signal, to a spatial separation of the various emitting species, and to optical depth measurements.

INSTRUMENT SPECIFICATIONS:

Imaging CCD spectrograph covering the $2,000-9,000 \AA$ range in two channels. Spectral resolution of 1-3 $\AA$. Spatial resolution during remote viewing (RV) phase about $10 \mathrm{~km}$ at closest approach from the nucleus. This instrument could be combined with the EUV spectrophotometer. 


\section{Magnetometer}

SCIENTIFIC OBJECTIVE:

The magnetometer will aid in (1) investigation of the nature of comet-solar wind interaction, (2) investigation of the magnetic field and electric current environment of cometary ionosphere and ion tail, and (3) investigation of the magnetic field of the cometary nucleus using fast electrons as remote sensing probe.

OPERATING PRINCIPLE:

Triaxial flux-gate magnetometer (or other types)

PRIMARY DATA:

Maximum sampling rate $\sim 30$ vectors per second; measuring ranges per component in nanoteslas: $\pm 16, \pm 64, \pm 256, \pm 1024, \pm 4096$, $\pm 16384, \pm 65536$; resolution in nanoteslas: $\pm 0.004, \pm 0.016, \pm 0.064$, $\pm 0.25, \pm 1, \pm 4, \pm 16$

HERITAGE:

Voyager, Giotto, and numerous other space missions

DEVELOPMENT STATUS:

Hardware fully developed

INSTRUMENT SPECIFICATION:

Mass: $2 \mathrm{~kg}$

Power: $2 \mathrm{~W}$

Data rate: $100-1,000$ bps

SUPPORTING SUBSYSTEMS:

Booms 


\section{Gamma-Ray Spectrometer}

SCIENTIFIC OBJECTIVE:

The gamma-ray spectrometer will investigate past and present state of planetary body as derivable from measurements of surface composition.

OPERATING PRINCIPLE:

Detection of gamma rays characteristic of particular elements PRIMARY DATA:

Pulse height spectra

HERITAGE:

Organic scintillers $\mathrm{NaI}(\mathrm{T} 1)$ spectrometer systems were successfully used on Apollo 15 and Apollo 16. NaI(T1) detector will be replaced by $\mathrm{Ge}(\mathrm{HP})$ detector. These solid state detectors have been used on astrophysical and solarphysics space flights and high-altitude balloon flight missions.

DEVELOPMENT STATUS:

Detector system: in test

Cooler: preliminary design

Electronics: largely to be adopted

INSTRUMENT SPECIFICATIONS:

Size: $29 \times 32 \times 50 \mathrm{~cm}$

Weight: $12 \mathrm{~kg}$

Power: $10 \mathrm{~W}$

Data rate: $1.5 \mathrm{kbps}$

SUPPORTING SUBS YSTEMS:

Coolers, booms, and shields 


\section{X-Ray Spectrometer}

SCIENTIFIC OBJECTIVE:

The $\mathrm{x}$-ray spectrometer will map elemental composition of rock and soil materials covering planetary surfaces and measure the interaction between the comet and solar environment.

OPERATING PRINCIPLE:

The $\mathrm{x}$-ray spectrometer measures fluorescence $\mathrm{x}$-rays emitted by elements in surface materials that are excited by solar $x$-rays. For the interaction of comets with the solar environment, spatial energy, and temporal distribution of the excited $x$-ray flux will be required. In this latter case, a telescope spectrometer will be needed.

PRIMARY DATA:

Primary data will include x-ray pulse height spectra from planetary surfaces. Geochemical maps will be obtained of the elements from $\mathrm{C}$ through Fe. Spatial, temporal, and energy distribution will be obtained in order to determine such interactions as x-ray Aurora, disconnection of comet-tail crossing sector boundaries, and shock-induced heating of the cometary ionosphere.

\section{HERITAGE:}

Apollo 15 and Apollo 16

Astrophysical and solar physics space flight missions

DEVELOPMENT STATUS:

Start the development of a combined $x$-ray and gamma-ray spectrometer system. Begin development of alternate solar monitor and use of $\mathrm{Si}(\mathrm{Li})$ or $\mathrm{HgI}$ detector.

INSTRUMENT SPECIFICATIONS:

These specifications are for a comet mission system measuring the energy spectrum from 0.2 to $12 \mathrm{KeV}$.

Size: $20 \times 20 \times 40 \mathrm{~cm}$

Weight: $11 \mathrm{~kg}$

Power: $10 \mathrm{~W}$

Data rate: $0.3 \mathrm{kbps}$

SUPPORTING SUBSYSTEMS :

Depending on whether solid state detectors are used and experiment combined with gamma rays

Cooler and boom mounted 


\section{Fast Ion Analyzer}

SCIENTIFIC OBJECTIVE:

The fast ion analyzer will study the solar wind plasma flow around the comet, (2) investigate the configuration of the cometary ionosphere and ion tails, and (3) study plasma processes (i.e., ionization and acceleration) in the cometary atmosphere.

OPERATING PRINCIPLE:

Electrostatic analyzer with good angular coverage (and possible addition of magnetic filter for mass separation)

\section{PRIMARY DATA:}

Two- to three-dimensional velocity distribution functions of solar wind plasma and cometary plasma $(10 \mathrm{eV}$ to $50 \mathrm{KeV})$ with some capability of composition analysis

HERITAGE:

Ampte, Giotto, and numerous other missions

DEVELOPMENT STATUS:

Flight ready for many solar wind plasma instruments (from ISEE, Helios, etc.) and near completion for Ampte and Giotto missions INSTRUMENT SPECIFICATION:

Mass: 3 kg

Power: $3 \mathrm{~W}$

Data rate: $300-1,000$ bps

SUPPORTING SUBSYSTEMS:

None 


\section{Electron Analyzer}

SCIENTIFIC OBJECTIVE:

The electron analyzer will (1) determine the structure and morphology of the cometary ionosphere and ion tail by measuring the electron component, (2) study ionization and acceleration processes in the cometary atmosphere, and (3) investigate the magnetic field environment of the cometary ionosphere and cometary nucleus using fast electrons as remote sensing probe.

OPERATING PRINCIPLE:

Electrostatic analyzer with good angular coverage and fast time resolution

PRIMARY DATA:

Two- to three-dimensional velocity distribution of electrons in the energy range of $10 \mathrm{eV}$ to $50 \mathrm{KeV}$.

HERITAGE:

Numerous magnetospheric and solar wind missions, Apollo, and Giotto

DEVELOPMENT STATUS:

Requirement of angular resolution in a three-axis stabilized spacecraft probably needs modification of previous designs.

INSTRUMENT SPECIFICATIONS:

Mass: $2 \mathrm{~kg}$

Power: $2 \mathrm{~W}$

Data rate: $300-1,000$ bps 


\section{Plasma Wave Analyzer}

SCIENTIFIC OBJECTIVE:

The plasma wave analyzer will (1) investigate the morphology of the cometary ionosphere and ion tail using plasma waves as diagnostic tools (i.e., determination of the total electron density and plasma instabilities), and (2) evaluate currents, wave-particle interactions, and acceleration processes in the cometary plasma environment.

OPERATING PRINCIPLE:

Magnetic field search-coil sensor and electric field sensor PRIMARY DATA:

E field: 16 channels, $18 \mathrm{~Hz}$ to $100 \mathrm{kHz}$

B field: 11 channels, $0.3 \mathrm{~Hz}$ to $1 \mathrm{kHz}$

HERITAGE:

Voyager, ISEE, and VEGA

DEVELOPMENT STATUS:

Hardware fully developed

INSTRUMENT SPECIFICATION:

Mass: $6 \mathrm{~kg}$

Power: $7 \mathrm{~W}$

Data rate: 500 bps

SUPPORTING SUBSYSTEMS:

Booms 


\section{Electromagnetic Sounder for a Cometary Nucleus}

The scientific justification for an active electromagnetic radio sounder (hereafter termed "sounder") is to probe the internal physical structure of the nucleus and determine if it is homogeneous, layered, or has a well-defined rocky core with an ice mantle. The sounder emits a short pulsed electromagnetic wave at the nearby nucleus and the transmitted wave propagates until partially reflected by any macroscopic change in the dielectric constant of the traversed medium. Thus, the first returning signal will come from the surface interface, and later returns will come from scattering centers and interfaces inside the nucleus. By directing the sounder pulses at different locations around the nucleus, a threedimensional map of the internal structure may be determined. Near surface sounding (depths of $100-200 \mathrm{~m}$ ) could be done from a range of $100 \mathrm{~km}$ for most icy materials. Deep sounding (more than a few hundred meters) requires a much closer range, preferably less than $10 \mathrm{~km}$. The cometary sounder instrument would be an extension of the technology developed for successful mapping of terrestrial glaciers by sounders carried in overflying aircraft. There is the possibility of combining the radar altimeter, required for navigation during close rendezvous, and the radio sounder. A range accuracy of $25 \mathrm{~m}$ could be met if an algorithm could be developed that would minimize the confusion between surface and subsurface echoes. However, the maximum range at which the nucleus could be detected would be only $400 \mathrm{~km}$.

The tabulation below gives the system parameters for twofrequency pulsed radio sounder. By spacecraft standards, the sounder is on the high end in terms of mass, power, and bit rate requirements for nonimaging instruments. A possible drawback would be the confusion that may arise in the interpretation of returning signals from a nucleus with an extremely rough surface. 
Frequency (MHz)

Wavelength (m)

Pulse length $(\mu \mathrm{s})$

Bandwidth ( $\mathrm{MHz}$ )

Range resolution (m)

In free space

In ice $(\epsilon=3.4)$

Peak power transmitted (W)

Average power transmitted (W)

Antenna field of view (deg.)

Mass (including antenna) $=10-13 \mathrm{~kg}$

Power $=20-25 \mathrm{~W}$

Bit rate $=16 \mathrm{kbs}$

Size $=30 \times 30 \times 10 \mathrm{~cm}$
Mode 1

60

5

5

6

25

15

500

0.25

$\pm 30$
Mode 2

180

1.66

1.5

20

7.5

4

500

0.07

$\pm \mathbf{3 0}$ 


\section{Test Mass Experiment}

The determination of the asteroid mass during a flyby cannot be achieved with sufficient precision (less than 5 percent) by simple tracking of the spacecraft from the Earth. The use of an external "gradiometer" is then needed.

It consists of the release of a small test mass (of the order of $20 \mathrm{~cm}$ in diameter) from the spacecraft a few days before the closest approach to the asteroid. The ejection angle and the speed relative to the main spacecraft have to be chosen so that the test mass passes closer to the asteroid are more perturbed by it than the spacecraft itself. As an example, an ejection angle of the order of $\mathbf{4 5}$ degrees from the spacecraft-asteroid direction and a relative speed of the order of $0.6 \mathrm{~m} / \mathrm{s}$ allow an accurate enough determination of the mass.

Just after the release of the test mass, the on-board radar altimeter and the camera will track it, in order to determine with the largest possible accuracy the relative speed and the position of the test mass on the star background. As a consequence of the different shape and mass of the probe and the spacecraft, the solar radiation pressure will perturb differently both objects. The effect must be taken into account during reduction of the data and is the main source of perturbation before the encounter. Regular observations of the test mass of the camera are then needed before the closest approach in order to predict the unperturbed path of the test mass on the star field.

After the closest approach, the camera must observe again the test mass. The angular displacement (of the order of some arc. min.) between the observed and the predicted position, together with the relative flyby velocity and the mass distance will give the mass of the asteroid with a convenient accuracy. With a typical example of a $50 \mathrm{~km}$ diameter asteroid, the mass can be evaluated with an accuracy of the order of 2 percent. 


\section{Radar Altimeter}

SCIENTIFIC OBJECTIVES AND OPERATING PRINCIPLES: The on-board radar altimeter will be used for navigation of the spacecraft within $750 \mathrm{~km}$ of the rendezvous target. The altimeter will directly support the target's mass determination experiment, provide spacecraft-object distances for the other scientific instruments, and characterize the roughness of the object's surface. The altimeter's heritage is PVO, Viking lander, and Seasat. The antenna ( $\sim 1-\mathrm{m}$ diameter) must be either located upon the scan platform or have an articulating mounting located on the spacecraft body. The altimeter determines the spacecraft-object range by measuring the round trip travel time of a pulsed signal.

TECHNICAL INFORMATION:

Mass: $12 \mathrm{~kg}$ (sensor) plus $3 \mathrm{~kg}$ (antenna)

Size (excluding antenna): $\sim 0.02 \mathrm{~m}^{3}$

Power: $\sim 20 \mathrm{~W}$ (peak power $\sim 100 \mathrm{~W}$ )

Wavelength: $3 \mathrm{~cm}$ ( $\mathrm{X}$ band)

Pointing accuracy required: $\pm 0.2^{\circ}$

Field of view: $\sim 2^{\circ}$ cone

Data rate: $\sim 200$ bps (1 pulse per $10 \mathrm{~s}$ )

Velocity resolution: $1 \mathrm{~m} / \mathrm{s}$ (differenced range)

Instrument operates in two modes:

Mode 1

Coarse range

Multiple pulse

Uncompressed

Spacecraft-object range $1-750 \mathrm{~km}$

Range resolution $300 \mathrm{~m}$
Mode 2

Fine range

Single pulse

Compressed

1-200 km

$23 \mathrm{~m}$ 


\section{Instrument Package for Asteroid Penetrators}

\section{Accelerometer}

The accelerometer is required for determination of subsurface material properties and stratigraphy. This instrument would sense the deceleration experienced by the penetrator forebody as it slows down and comes to rest beneath the surface. Variations in material properties would appear as irregularities in the deceleration record. An instrument of this type is also required to determine the depth of penetration. Accelerometers of the type required exist and have been extensively tested.

\section{Chemical Analysis (Elemental Abundance)}

Two in situ elemental analysis systems have been studied for potential use on penetrators. They are the alpha backscatter and $\mathbf{x}$-ray fluorescence analysis and the neutron/gamma-ray analysis. Alpha Backscatter and X-Ray Fluorescence. An alpha backscatter instrument for a penetrator system would consist of an alpha source such as $242-\mathrm{Cm}$ with a collimator. Measurements are made of the elastically backscattered alpha particles; alpha particle excited protons $(\alpha, p)$ measurements are used to detect the abundances of $\mathrm{Na}, \mathrm{Mg}, \mathrm{Al}$, and $\mathrm{S}$, and x-ray fluorescences should detect elements heavier than $\mathrm{Mg}$. Three separate detectors are required for the particles, protons, and x-rays. The major problem facing the alpha backscatter experiment is associated with the contamination of material up to $2 \mathrm{~mm}$ from the penetrator during penetrator emplacement. Samples must be brought into close proximity to the excitation source and detector. Thus the simplest sampling technique would be to open a small port in the penetrator hull and irradiate the material directly adjacent to the hull. In order to obtain the most useful data, it would be necessary to employ a drill to penetrate the narrow contaminated zone and bring a sample of fresh material into the source zone.

A second problem concerns the x-ray detector. A number of $\mathbf{x}$ ray detectors for nondispersive analysis are available; most require cryogenic cooling. Passive cooling systems are now being designed but have not been considered for use on penetrators. Detectors such as $\mathrm{HgI}$, which do not require cooling, are presently under development, and they may be available for penetrator missions. 
Neutron/Gamma-Ray Analysis. The neutron gamma-ray analysis system consists of a machine pulsed neutron excitation source and a nondispersive gamma-ray spectrometer. Measurements are in two modes. Prior to the initiation of neutron irradiation, gamma-ray spectral measurements of the natural background are made. This background consists of gamma-ray emission from the natural radioactive emitters $K, T h$, and $U$ and cosmic-ray induced gamma-ray emission. An analysis of the discrete line spectra will yield information concerning the abundances of $\mathrm{K}, \mathrm{Th}, \mathrm{U}, \mathrm{H}, \mathrm{O}$, $\mathrm{Si}$, and possibly $\mathrm{Fe}$ and the lanthanides. If a $14 \mathrm{MeV}$ pulsed neutron source is used in the second neutron production phase, then information concerning the element abundances of $\mathrm{H}, \mathrm{O}, \mathrm{Si}$, $\mathrm{Mg}, \mathrm{Fe}, \mathrm{Ti}, \mathrm{S}, \mathrm{C}, \mathrm{Ca}, \mathrm{Mn}$ and possible other minor element constituents will be obtained. The extent to which these elements can be detected strongly depends on the detector used. The best neutron source for this project is the pulsed neutron generator using a $(\mathrm{D}, \mathrm{T})$ reaction yielding $14 \mathrm{MeV}$ neutrons of $\sim 10^{6}$ neutrons per pulse. The gamma-ray detector is a major problem. High-resolution detectors require cooling. Passive cooling modes can be developed and, thus, reduce the magnitude of the problem. Even if passive cooling is not available, $\mathrm{NaI}(\mathrm{T} 1)$ detectors can be used, but there is a decrease in sensitivity. The neutron generators have the capability of surviving the impact penetrator environment. The gamma-ray detectors need to be tested further. A great advantage of the neutron/gamma-ray system is that it requires no sample preparation. The measurements yield information concerning the bulk elemental abundance $\sim 16$ in diameter sphere about the gamma-ray detector. Average bulk density can also be determined.

Imaging

One of the most promising systems for a penetrator would be a small charged-coupled device (CCD). The camera would be mounted on the highest part of the penetrator afterbody. One possible configuration would have several detectors fixed in position and viewing in different directions. An alternative scheme may involve a single detector that is rotated by a small motor or clockwork device, this latter development of a rotation system capable of withstanding high $\mathrm{g}$-loads experienced by the afterbody. CCD arrays have been successfully tested to high g-loads. 


\section{ACKNOWLEDGMENTS}

The study group could not have accomplished its task without the able support of the following people: Angelo Atzei, ESTEC; John Beckman, JPL; Geoffrey Briggs and Al Diaz, NASA Headquarters; George Haskell, ESA Headquarters; Kerry T. Nock, JPL; John Niehoff, SAI; Ernst Stuhlinger, MPI; and Chen-wan L. Yen, JPL. We are grateful for their many significant contributions. It is particularly appropriate to single out for special thanks Ernst Stuhlinger, who had the idea of the primitive bodies grand tour, and Chen-wan Yen, who gave it substance in terms of attractive mission targets. 


\section{REFERENCES}

Blanchard, M. B., V. R., Oberbeck, T. E. Bunch, R. T. Reynolds, T. N. Canning, and R. W. Jackson, 1976. NASA TM X-73, 181 pp.

Festou, M. In press. Astronomy and Astrophysics

IAU Circular 3501

IAU Circular 3528

IAU Circular 3531

IAU Circular 3534

Murphy, J. P., R. R. Reynolds, M. B. Blanchard, and U. S. Clanton 1981.

NASA TM 81251

Niehoff, J. 1978. Asteroids: An exploration assessment NASA Conf. Publ. 2053 ,

Sekanina, Z. 1979. Icarus 47: p. 420

Turkevich, A., and T. Economou, Asteroids: An Exploration Assessment NASA Conf. Publ. 2053, 1978.

Vsekhavyaksky 1964. NASA TT F 80

Yeomans, D. 1974. PASP, 86: 125 